NBER WORKING PAPER SERIES

\title{
INDUSTRY INPUT IN POLICYMAKING: EVIDENCE FROM MEDICARE
}

\author{
David C. Chan, Jr \\ Michael J. Dickstein \\ Working Paper 24354 \\ http://www.nber.org/papers/w24354 \\ NATIONAL BUREAU OF ECONOMIC RESEARCH \\ 1050 Massachusetts Avenue \\ Cambridge, MA 02138 \\ February 2018
}

\begin{abstract}
We are thankful to Dan Barron, Panle Barwick, Steve Callander, Alice Chen (discussant), Jeff Clemens, Zack Cooper, David Cutler, Wouter Dessein, Liran Einav, Ray Fisman, Bob Gibbons, Ben Golub, Josh Gottlieb, Matt Grennan (discussant), Jon Gruber, Wes Hartmann, Alex Hirsch, Zachary Hochstetler, Matt Jackson, Kei Kawai, Dan Kessler, Amanda Kowalski (discussant), Keith Krehbiel, Jon Levin, Danielle Li (discussant), Shih En Lu, Ateev Mehrotra, Joe Newhouse, Mike Powell, Jim Rebitzer (discussant), Al Roth, Ken Shotts, Sherry Smith, Bob Town (discussant), Francesco Trebbi, Noam Yuchtman, multiple members of the RUC who participated in detailed interviews, and many seminar participants. Sam Arenberg, Lulua Bahrainwala, Peter Favaloro, Atul Gupta, Johnny Huynh, Michael Kobiela, Douglas Laporte, and Lindsay Yang provided excellent research assistance. Chan gratefully acknowledges support from NIH DP5OD019903-01, NIH L30 AG051189-01, and NIH P30AG012810. The views expressed herein are those of the authors and do not necessarily reflect the views of the National Bureau of Economic Research.
\end{abstract}

NBER working papers are circulated for discussion and comment purposes. They have not been peer-reviewed or been subject to the review by the NBER Board of Directors that accompanies official NBER publications.

(C) 2018 by David C. Chan, Jr and Michael J. Dickstein. All rights reserved. Short sections of text, not to exceed two paragraphs, may be quoted without explicit permission provided that full credit, including $\odot$ notice, is given to the source. 
Industry Input in Policymaking: Evidence from Medicare

David C. Chan, Jr and Michael J. Dickstein

NBER Working Paper No. 24354

February 2018

JEL No. D71,H57,I13,L51

\section{ABSTRACT}

In setting prices for physician services, Medicare solicits input from a committee that evaluates proposals from industry. We investigate whether this arrangement leads to prices biased toward the interests of committee members. We find that increasing a measure of affiliation between the committee and proposers by one standard deviation increases prices by $10 \%$, demonstrating a pathway for regulatory capture. We then evaluate the effect of affiliation on the quality of information used in price-setting. More affiliated proposals produce less hard information, measured as lower quality survey data. However, affiliation results in prices that are more closely followed by private insurers, suggesting that affiliation may increase the total information used in price-setting.

David C. Chan, Jr

Center for Health Policy and

Center for Primary Care and Outcomes Research

117 Encina Commons

Stanford, CA 94305

and NBER

david.c.chan@stanford.edu

Michael J. Dickstein

New York University

Stern School of Business

Kaufman Management Center,7-78

44 West 4th Street

New York, NY 10012

and NBER

michael.dickstein@nyu.edu 


\section{Introduction}

In regulation and procurement, governments often face an information deficit. Industry participants know much more about key inputs for policy decisions, such as production costs, but have incentives to provide selected or distorted information to direct policy in their own interests. Thus, obtaining valuable information from industry to make policy decisions may also provide a general pathway for "regulatory capture," potentially biasing government decisions toward an industry's preferred policies (Stigler, 1971; Peltzman, 1976). Understanding and measuring this trade-off between better information collected for decision-making and the distortion from regulatory capture seems particularly relevant given the US government's reliance on hundreds of advisory committees in many important policy decisions. ${ }^{1}$

Our empirical work focuses on the US government's procurement of health care services. Medicare, the federal health insurance program for the elderly, sets administered prices for the roughly $\$ 70$ billion in annual payments it allocates for physician services. ${ }^{2}$ To do so, the government relies on a committee of physicians convened by the American Medical Association (AMA), known as the Relative Value Scale Update Committee (RUC). The committee evaluates proposals from specialty societies to determine the relative resource costs of services. The committee's recommendations influence not only Medicare's direct expenditures, but also indirectly shape pricing in the overall market for physician services, valued at $\$ 480$ billion per year or $2.7 \%$ of the US GDP (Clemens and Gottlieb, 2017). The prices of medical procedures can also drive larger changes in physicians' procedural choices (Clemens and Gottlieb, 2014; Gruber et al., 1999) and the specialty career decisions of future physicians (Nicholson and Souleles, 2001).

We first assess whether the composition of the RUC leads to prices biased in favor of its members, a concern raised by observers of this important committee (Laugesen, 2016). Using novel data from the RUC on the universe of price-setting proposals discussed between 1992 and 2013, we focus on the RUC's primary role of assessing the work involved for the service in

\footnotetext{
${ }^{1}$ See Brown (2009) for an introduction. In 1972, Congress enacted the Federal Advisory Committee Act to track the existence of a large number of federal advisory committees. In 2006, the US government maintained 916 such committees, with 67,346 members, at a cost of $\$ 384$ million. While advisory committees may serve to improve the quality of policy decisions, a key challenge for maintaining such committees is to ensure they are "fairly balanced" and free of "inappropriate influence."

${ }^{2}$ Medicare payments to physicians totaled $\$ 70$ billion in 2015 , and the US Congressional Budget Office projects spending of $\$ 82$ billion in 2020, and $\$ 107$ billion in 2025 (Congressional Budget Office, 2016).
} 
each proposal and assigning a work-based relative value. ${ }^{3}$ To measure the effect of connections with the RUC, we develop a measure of affiliation, to reflect the closeness in global preferences between specialties with many interests. Our measure exploits data on the services each speciality performs, in a way that we show reflects the likelihood that the revenue of two specialties will covary under any set of price changes. We then examine whether proposals by specialty societies with higher affiliation with the RUC receive higher prices.

We find that increasing a proposal's affiliation by one standard deviation increases its price by $10 \%$. To interpret this relationship as causal, we rely on two sources of identifying variation. First, the composition of RUC voting members changes across meetings, as the RUC has expanded voting seats over time and as some specialty seats explicitly rotate. Second, and to a larger degree, the specialties proposing to the RUC for a given procedure may vary widely. To address the concern that the underlying price of a procedure may be correlated with affiliation, either through the RUC's composition or through the procedure's likely proposers, we control for RUC meeting identities and the shares of a procedure's Medicare utilization across specialties. The remaining variation, from plausibly idiosyncratic costs of proposing and from barriers to coordination among many potential proposers, may generate different levels of affiliation for procedures with the same intrinsic price. We provide support for this identifying assumption by showing that, after controlling for meeting identities and specialty shares, the residual affiliation is uncorrelated with exogenous measures of a service that predict its price.

Our use of quasi-random variation in proposer identities complements previous research on committees, which typically exploited the rotation of committee members (Zinovyeva and Bagues, 2015; Li, 2017; Camara and Kyle, 2017). Our new source of variation aligns with a mechanism from the literature on lobbying (Bertrand et al., 2014; Blanes i Vidal et al., 2012): The identity of the lobbyist representing a special interest may be valuable because it lends credibility to the interest's proposal, particularly when the lobbyist's preferences align with those of the decision-maker (Kessler and Krehbiel, 1996; Hirsch and Montagnes, 2015).

\footnotetext{
${ }^{3}$ The work-related component of relative prices have received the most policy and research attention (e.g., Bodenheimer et al., 2007; Sinsky and Dugdale, 2013; Laugesen, 2016). According to the AMA (2017), this component equals $51 \%$ of overall reimbursement. Two other components of relative price are professional liability insurance (4\%) and practice expenses (45\%) (e.g., ancillary staff labor, supplies, and equipment). The RUC also determines the practice expense component, but via a separate process. We provide more details in Section 2.
} 
Because specialties have multiple, sometimes shared interests, the effect of affiliation on specialty revenue requires careful analysis. We show in a counterfactual calculation that if affiliation were equalized across proposals and if Medicare's budget remained fixed, roughly $1.9 \%$ of revenues would be reallocated across specialties, representing about $\$ 1.3$ billion in annual Medicare spending or $\$ 8.9$ billion in annual health care spending accounting for both Medicare and private insurance. Unpacking this average level of reallocation, however, we observe distributional consequences by specialty. Emergency medicine would have the largest percentage revenue gain $(+17 \%)$ from equalizing affiliation, while infectious disease would have the largest loss $(-5.8 \%)$. Interestingly, specialties like internal medicine and family medicine are net beneficiaries of affiliation, because they share many services in common with RUC member specialties, including the standard office visit. More than doubling the number of internal medicine seats on the RUC would increase the specialty's revenue by less than $1 \%$.

The possibility of bias in the RUC's decision-making raises a central question of regulatory design: From the government's perspective, what is the offsetting value of inviting industry input in policymaking? In settings involving advisory committees, a key feature is the importance of policy-relevant knowledge (e.g., the safety and efficacy of a drug, or the benefits and costs of electricity generation) held by industry participants. The government may form advisory committees with members that hold such knowledge directly. In other cases, committee members may lack direct knowledge, but instead have the task of extracting and synthesizing information from outside special interests. We thus explore whether allowing some bias in advisory committees may improve regulatory decisions, by facilitating the communication of information that is neither verifiable nor independently discoverable. In our setting, we explore whether Medicare can extract more information about physician services and set more appropriate prices by employing the RUC as an intermediary in decision-making.

To address this question, we begin with a conceptual model, borrowing ideas from a large literature on the extraction of information from biased experts. ${ }^{4}$ Following this literature, we model two types of information that the government wishes to extract. If information is soft, or

\footnotetext{
${ }^{4}$ See Grossman and Helpman (2001) for an extensive review. Some prominent examples of papers in this large literature spanning political science and economics include Crawford and Sobel (1982), Calvert (1985), Austen-Smith (1994), Dewatripont and Tirole (1999), and Li et al. (2001).
} 
unverifiable, it must be credibly communicated (Crawford and Sobel, 1982). The government may then benefit from delegating decision-making to an intermediary (the RUC) that has preferences closer to the biased expert (the specialty), because aligned preferences improve communication (Dessein, 2002). On the other hand, a committee with adversarial preferences incentivizes the specialty to generate more information that is hard, or verifiable (Dewatripont and Tirole, 1999; Hirsch and Shotts, 2015). The net effect thus depends on the nature of information relevant for decisions. In the Medicare setting and many others, some information (e.g., the average time for physicians to perform a service) is conceivably verifiable, but much (potentially most) of the relevant information is difficult to verify and therefore soft (e.g., the "difficulty" or "complexity" of a service relative to another).

We then test the predictions of this model of information extraction using two unique and objective measures of information quality. First, we test for the effect of greater affiliation on hard information using the quality of survey data presented to the RUC. Consistent with our model, we find that higher affiliation corresponds to less hard information, in that proposals submitted to a RUC with greater affiliation feature fewer physicians surveyed and fewer respondents, conditional on specialty shares and other proposal and procedure characteristics. Also consistent with the theory, greater hard information, conditional on affiliation, is not correlated with higher prices. Thus, we find empirical support for the theoretical notion, as in Aghion and Tirole (1997), Dewatripont and Tirole (1999), and Hirsch and Shotts (2015), that separation in interests can provide motivation for an agent to provide costly but valuable information to a principal.

Second, to examine a policy-relevant metric of the overall level of (hard and soft) information Medicare collects through the RUC, we measure the degree to which Medicare price changes correlate with private insurance price changes (Clemens and Gottlieb, 2017; Clemens et al., 2017). We classify price changes depending upon whether they originate from RUC decisions, and if so, whether they originate from high- vs. low-affiliation proposals. We find that price changes in private insurance track those changes in Medicare more closely when the Medicare price changes arise from RUC decisions. Further, we find stronger price-following for Medicare price changes arising from more highly affiliated proposals to the RUC, relative to price changes from low-affiliation RUC proposals. These findings suggest that affiliation may improve the 
overall quality of information in Medicare pricing decisions.

We organize the remainder of the paper as follows: Section 2 describes the institutional setting. Section 3 introduces our data, measure of affiliation, and discusses our identification strategy. Section 4 presents our main results on the effect of affiliation on relative prices and discusses our interpretation of bias. We move to the question of information extraction in Section 5. We introduce a theoretical framework and then present empirical evidence using data on survey quality and on the transmission of Medicare prices to private insurance prices. Section 6 concludes.

\section{Institutional Setting}

We study the price-setting mechanism within Medicare's Part B, which finances physician and other clinical services as part of the federal health insurance program for the elderly. While in private insurance, providers may negotiate prices directly with payers (Lewis and Pflum, 2015; Ho and Lee, 2017), Medicare sets its prices using an administrative formula. This arrangement is similar to price cap rules in regulated industries, including telephone service in past decades (e.g., Braeutigam and Panzar, 1993), and to fee schedules for medical care in other countries. Similar to these other regulated settings, Medicare's formula attempts to set payments according to the costs and effort necessary to perform a service.

To tie payments to costs, Medicare measures the level of costs for a service by summing three distinct components: the intensity and effort of the physician's work $(W)$, the practice expense required to perform the service $(P E)$, and the professional liability insurance physicians must carry $(P L I)$. Each element has its own relative price, known as a "relative value unit," or RVU. The payment levels adjust for differences in the cost of practicing medicine in different parts of the country. To convert the relative value units into dollars, the sum of the (geographically adjusted) cost components is multiplied by a common conversion factor; in 2014, the conversion factor was approximately $\$ 35.83$ per RVU (American Medical Association, 2015). ${ }^{5}$

\footnotetext{
${ }^{5}$ The conversion factor is set administratively so that Medicare's total payments for procedures in the US falls within a budget determined by factors such as GDP growth and the number of Medicare beneficiaries. We provide more details in Appendix A-1.
} 
In notation, for each service $i$ performed in geographic area $j$ in year $t$,

$$
\text { Reimbursement }_{i j t}=\left[\sum_{c \in\{W, P E, P L I\}}\left(\mathrm{RVU}_{i t}^{c} \times \mathrm{GPCI}_{j}^{c}\right)\right] \times \mathrm{CF}_{t} .
$$

where $\mathrm{RVU}_{i t}^{c}$, is the relative value unit for service $i$ in year $t, \mathrm{GPCI}_{j}^{c}$ is the fixed geographic practice cost index, and $\mathrm{CF}_{t}$ is the conversion factor. ${ }^{6}$

With the adoption of this formula, Medicare's administrators also created for themselves a new and complex task: determining the relative values or RVUs. Judging the level of effort required for each medical procedure requires collecting information possessed by actual practitioners. Medicare thus engages with a committee of the American Medical Association (AMA) to collect physicians' evaluations of the relative effort and advise on proper RVU levels. This committee - the RUC - recommends relative values to Medicare, which Medicare's administrators adopt over $90 \%$ of the time (American Medical Association, 2017; Laugesen et al., 2012).

\subsection{The RUC}

The RUC considers evidence and makes recommendations for both the work and practice-expense RVU components of the reimbursement formula, which together account for $96 \%$ of total RVUs. We focus on work RVUs, which account for the majority of total RVUs across services and have been the focus of increasing scrutiny. ${ }^{7}$ We henceforth use the term "RVU" or "relative price" interchangeably with "work RVU," unless otherwise specified.

The main RUC committee, currently comprised of 25 physician specialty society representatives, considers all changes to work RVUs. Twenty one of these members occupy permanent

\footnotetext{
${ }^{6}$ Medicare adopted this formula in 1992 (Hsiao et al., 1988). Prior to the current method, Medicare reimbursements were ill-defined and based on "usual and customary charges" that prevailed in each local (usually state-based) insurance market as administered by the state Blue Cross Blue Shield insurer. These prices resulted from negotiations between providers and insurers; they were thought to unfairly compensate certain specialists and also contribute to rising Medicare spending (Laugesen, 2016).

${ }^{7}$ The medical and health policy literatures have raised several potential sources of bias in the price-setting process, although largely descriptively and without access to the data contained in RUC proposals (e.g., Bodenheimer et al., 2007; Sinsky and Dugdale, 2013; Berenson and Goodson, 2016). The popular press has raised some of the same points (e.g., Whoriskey and Keating, 2013; Pear, 2015), and the Affordable Care Act explicitly funded more systematic evaluations comparing external measures of physician time (work) and Medicare-adopted measures (Wynn et al., 2015; Zuckerman et al., 2016). Recent work by Fang and Gong (2017) take stated times to perform certain services as explicit and connect these times with work RVUs in order to detect physician over-billing.
} 
seats, while the remaining four rotate. ${ }^{8}$ For example, a representative of the specialties of internal medicine, dermatology and orthopedic surgery maintain permanent seats, while specialities including pediatric surgery and infectious disease rotate on and off the RUC. In Table 1, we record the number of total meetings at which a particular specialty society had a voting member on the RUC. Clear from this count, many specialties have had a representative on the RUC since its founding in 1992, and some have had two representatives. In Figure 1, we show the number of voting seats and a breakdown between "cognitive" and "procedural" specialties over time. ${ }^{9}$ Using our definition, procedural specialties - i.e, those who chiefly carry out surgical services - have a slightly larger share of the RUC's voting members in every year since 1992. The composition of the RUC has changed over time both because some of the seats explicitly rotate and because the committee size has grown over time.

\subsection{The Price-Setting Process}

Each year, in three meetings, approximately 200-300 physician services appear for review before the RUC. The committee will review all newly created services and will re-evaluate some existing services. Evaluations for existing services occur when the description or content of the procedure itself changes, when Medicare requests a revaluation, and, since 2006, when a workgroup from within the RUC identifies a service as potentially misvalued. ${ }^{10}$ In addition, The Omnibus Budget Reconciliation Act of 1990 requires Medicare's administrators to review relative values at least every five years, collecting public comments on potentially misvalued codes. The RUC has advised Medicare in these "Five-Year" reviews, evaluating 1118 services in 1997, 870 codes in 2002, 751 codes in 2007, and 290 additional codes in 2012 (American Medical Association, 2014).

\footnotetext{
${ }^{8}$ The rotating seats include two from internal medicine subspecialties not on the RUC, one primary care rotating seat, and one seat from a speciality society that is not a permanent member of the RUC and not eligible for one of the other three rotating seats. In addition, there are three voting seats that are not held by physician specialties (American Medical Association, 2017).

${ }^{9}$ Although the labels "procedural" and "cognitive" have been used frequently to describe specialties in the policy debate on the RUC (see, e.g., Berenson and Goodson, 2016), there is no set categorization of specialties according to these labels. We assign these labels to specialties based on conversations with the RUC. We provide more detail in the note to Figure 1.

${ }^{10}$ The RUC's Relativity Assessment Workgroup identifies potentially misvalued services by objective screens, such as when physicians bill for a service with low work RVUs in multiple units per patient, or when a service that physicians commonly performed in inpatient settings moves to the outpatient setting (American Medical Association, 2014). Specialties may also appeal to Medicare to request that the RUC review a service; such specialty requests represent a small minority of cases.
} 
For each code under review, the evaluation process begins by identifying a specialty or set of specialties to collect evidence and propose an RVU to the RUC. Any of the 122 specialty societies in the American Medical Association's House of Delegates may weigh in on the development of an RVU proposal, but typically only those who perform the service will volunteer to collect evidence and contribute to the proposal. We later exploit variation in the exact composition of the proposing group in our empirical analyses.

Briefly, the process from proposal to approval involves the following steps:

1. The specialities developing a proposal conduct a survey of their members to collect data about the work and resource use involved in the given service.

(a) If surveying, specialties decide on the number of physician members to survey. Physicians are asked to compare the service with "reference services" and to give estimates of the time and other measures of work required (e.g., mental effort, technical skill, psychological stress). The survey contains a standardized vignette for the service, to ensure consistency of the estimates.

(b) The one or more specialties who have conducted surveys present their evidence and arguments for a proposed relative price before the RUC.

2. The RUC members discuss the proposal with each other and with the proposer(s). Proposals pass with at least a two-thirds vote of the committee. ${ }^{11}$

3. The RUC forwards its recommendations to Medicare, which historically accepts the relative prices 90\% of the time (American Medical Association, 2017; Laugesen et al., 2012). Medicare, using formulas in Equation (1) and Appendix A-1, translates these relative prices into payment levels in dollars.

\footnotetext{
${ }^{11}$ If a proposal is not approved, the proposer(s) may discuss their proposal with a smaller "facilitation committee". In facilitation, the proposed value is often revised downward. The RUC must still pass any revisions. The RUC may also independently recommend a relative price to Medicare if no proposal is successful.
} 


\section{Empirical Approach}

We analyze the RUC's role in the price-setting process using data from the committee's deliberations. Our substantive goals are twofold. First, we measure the causal effect of the RUC's affiliation with the proposing specialities on the prices recommended by the committee. Second, we determine the effect of affiliation on information transmission, measured either as survey precision (hard) or the correlation between price changes in Medicare and in private insurance (hard and soft). To do so, we need to define an empirical measure of affiliation, and then describe the plausibly exogenous variation in this affiliation that allows us to identify the casual effect of affiliation on prices and on information transmission.

\subsection{Data}

Our empirical analyses rely on three sources of data. First, we use information on the RUC's deliberations, including the RUC membership at each decision and the details of the proposal for each service evaluated by the committee. We accessed the same database RUC members use to prepare for votes during meetings, with detailed proposal information for each service the RUC evaluated from its inception in 1992 until 2013. For each proposal, we collect the identity of the service, the meeting in which the RUC considered the proposal, the specialty society or societies involved, the RVU level proposed, and the RVU level recommended by the RUC. We observe 4,423 proposals with known specialty proposers and other selection criteria. We describe details of our sample creation in Table A-1.

The RUC's database also contains detailed characteristics of each proposal. We observe the characteristics of the survey, a central component of proposals, including the number of physicians surveyed and the number of respondents. We also collect summary statistics of the survey responses regarding the time required for a service, as well as comparisons between the service and a "reference" service along various qualitative dimensions (e.g., complexity of medical decision-making, urgency, technical skill, physical effort). ${ }^{12}$

Second, in addition to the RUC database, we collect objective measures of the characteristics

\footnotetext{
${ }^{12}$ In the survey questions on time, we observe time information broken into preparation time before the procedure (median), the time for the actual service itself (25th, 50th, and 75th percentiles), any post procedure time, and indicators for whether surgical procedures require additional office visits before or after the surgery.
} 
of each service to use as controls in our analyses and to identify the types of physician specialities that use each code. The data come from Medicare, including its annual utilization files and a survey of Medicare beneficiaries. With these data, we define a set of service-specific characteristics, including: (i) yearly Medicare utilization of a given service, broken out by the identity of the specialty providing the service; (ii) average demographics of patients who receive a given service; and (iii) the fraction of utilization of the service in different medical settings, including the emergency department, inpatient, outpatient care settings.

To build even more detailed control variables to characterize each service, we merge in a database of service descriptions. ${ }^{13}$ The description field includes a set of words that Medicare, other payers, and clinicians use to categorize physician work for reimbursement and productivity measurement. We identify keywords from this collection of descriptive terms and create variables that reflect a service's description. ${ }^{14}$

Finally, third, we collect a time series of private sector prices for each service. We later compare the changes in private prices to those in Medicare, to explore how private insurers respond to information and possible bias in Medicare's price setting mechanism. We use Truven Health's MarketScan data to measure prices for each service as paid by private insurers. We observe quantities of use, the specialty of the billing physician, and a measure of the reimbursement paid to the provider. We scale the Marketscan data by patient demographics in the Medical Expenditure Panel Survey (MEPS) dataset, to find nationally representative estimates of private insurance utilization for each procedure and for each specialty performing it.

\subsection{Affiliation}

We define a notion of affiliation to measure the alignment of preferences between specialties with a diverse set of interests, characterized by the services they each perform. ${ }^{15}$ Two specialties may be tightly linked, even if they are discussing a procedure only one performs. For example,

\footnotetext{
${ }^{13}$ In Table A-2, we provide examples of these descriptions.

${ }^{14}$ In detail, we identify word stems to account for inflected variations (e.g., "operate" and "operation"), of which there are a total of 9,271 unique stem words from 11,123 original words, excluding stop words such as "the," "and," and "only." The median count of unique word stems across procedure code descriptions is 8, and the 5th and 95th percentiles are 3 and 22 , respectively. We use these word stems to create a vector of indicator variables reflecting the content of a service's description field.

${ }^{15}$ This concept is similar to congruence in Caillaud and Tirole (2007), which they define as the "prior probability that a given member benefits from the sponsor's project."
} 
suppose the orthopedic surgery specialty proposes a value for a spinal surgery, and this service represents only a small fraction of its specialty revenue. If hand surgery as a specialty holds a committee seat, it may tend to agree with an orthopedic surgery recommendation, even if it performs no spinal procedures, because it shares other procedures in common with orthopedic surgery. The alignment of preferences (or lack thereof) will have implications for both the prices set and the information transmitted in the process, as we detail later in Section $5 .{ }^{16}$

To measure alignment in specialty preferences, we must capture the many pathways through which price-setting decisions may affect a specialty's revenue (or any other function of quantities and prices of services). For example, changing the RVU of a service may affect the quantities of that service and of complementary or substitute services. Changes in quantities or RVUs will also affect the conversion factor and therefore the real Medicare reimbursement for any service. Finally, a price change may cause future prices of related services to change similarly, particularly for services that might use the index service as a reference. These mechanisms are complicated. However, by construction, two specialties with identical quantity shares across services will experience the same proportional change in revenue from any arbitrary price change. Thus, we focus on the similarity of services between two specialties, as a measure of the alignment in interests when specialties seek to maximize revenue.

Specifically, we denote the quantity utilization of service $i$ by specialty $s$ in year $y$ as $q_{i s y}$. We then construct the vector $\sigma_{s}$ of specialty $s$ utilization shares across all services, where the $i^{\text {th }}$ element is $\sigma_{i s}$, the share of specialty $s$ 's utilization of $i$ averaged across years:

$$
\sigma_{i s}=\frac{\sum_{y} q_{i s y}}{\sum_{y} \sum_{i} q_{i s y}},
$$

for each service $i$ in the universe of 11,252 Current Procedural Terminology (CPT) codes that physicians perform for reimbursement. We define affiliation between two specialties $s$ and $s^{\prime}$ as

\footnotetext{
${ }^{16}$ We thus depart from previous empirical work on committee bias that is based on financial ties (Camara and Kyle, 2017) or group membership (Zinovyeva and Bagues, 2015; Li, 2017), which focuses on bias and ex ante information. By measuring connections between senders (proposers) and receivers (committee members), using variation that is independent of the object of the proposal, we examine the relationship between preferences and communication (or ex post information extracted from the sender).
} 
a negative Euclidean distance:

$$
a\left(s, s^{\prime}\right)=-\sqrt{\left(\sigma_{s}-\sigma_{s^{\prime}}\right)\left(\sigma_{s}-\sigma_{s^{\prime}}\right)^{\prime}}
$$

In Appendix A-2, we provide further discussion about this measure of affiliation as our preferred statistic for the alignment of revenue maximization objectives between two specialties. ${ }^{17}$

Figure 2 shows affiliation measures between specialties, among the 20 specialties with the highest revenue, where we divide the measures into nine bins. Many affiliation measures are intuitive: We find high affiliations for related pairs such as between internal medicine and family medicine, between electrodiagnostic medicine and neurology, and between orthopedic surgery and hand surgery. Perhaps surprisingly, internal medicine is affiliated with many surgical specialties because many surgical specialties also rely on the same evaluation and management procedures that internal medicine uses. ${ }^{18}$ In contrast, physicians in pathology use a set of codes rarely used by other specialties, leading to low affiliations. Similarly, emergency medicine physicians provide evaluation and management services using distinct codes specific to emergency patients, and thus have low affiliations.

Our definition of affiliation reflects pairwise comparisons of the similarity in procedure use between two specialties. However, for our eventual empirical specifications, we need an affiliation measure at the proposal level, since our outcomes measures are specific to a proposal. Thus, we define set affiliation, a measure of affiliation between the set of specialities composing the RUC and the set of specialties party to a proposal. ${ }^{19}$ The set affiliation between the set of proposing specialties $S_{i}$ for proposal $i$ and the set of RUC member specialties $\mathcal{R}_{t}$ at meeting $t$ is

$$
A^{*}\left(\mathcal{R}_{t}, \mathcal{S}_{i}\right)=\frac{1}{\left\|\mathcal{R}_{t}\right\|} \sum_{r \in \mathcal{R}_{t}} \max _{s \in \mathcal{S}_{i}} a(r, s),
$$

\footnotetext{
${ }^{17}$ In particular, we discuss the use of quantity shares vs. revenue shares as the vector space upon which to base affiliation, and we discuss alternative distance metrics, such as Manhattan distance, correlation, and angular distance. Although there are theoretical reasons to prefer our chosen affiliation measure, we nevertheless show in Table A-3 that the affiliation effect on prices is robust across other formulations.

${ }^{18}$ Many important linkages between seemingly disparate specialties exist: Bronchoscopy is shared by otolaryngology, pulmonary medicine, and thoracic surgery. Plain x-rays are shared between internal medicine, radiology, and surgery. CT scanning of the head is shared by radiology, neurosurgery, and neurology.

${ }^{19}$ Proposing coalitions exist in our sample. Of the 4,423 proposals in our baseline sample with known proposing specialties, $63 \%$ are made by a single specialty, $23 \%$ are made by two specialties, and $14 \%$ are made by three or more specialties.
} 
where $r \in \mathcal{R}_{t}$ denotes a member specialty on the RUC, and $s \in \mathcal{S}_{i}$ denotes a specialty on the proposal. For each $r \in \mathcal{R}_{t}$, we take the maximum affiliation between $r$ and any proposing specialty $s \in \mathcal{S}_{i}$. In this formulation, additional proposing specialties in $\mathcal{S}_{i}$ can only increase $A^{*}\left(\mathcal{R}_{t}, \mathcal{S}_{i}\right)$, based on the intuition in Krishna and Morgan (2001) that communication outcomes improve when a receiver listens to the most closely aligned sender. We then take the average across RUC members, to reflect that the RUC aggregates opinions across members, not only in voting but also in the committee's private and public discussions ( $\mathrm{Li}$ et al., 2001). Finally, for interpretation, we standardize $A^{*}\left(\mathcal{R}_{t}, \mathcal{S}_{i}\right)$ by subtracting the sample mean and dividing by the sample standard deviation, and denote this standardized measure as $A\left(\mathcal{R}_{t}, \mathcal{S}_{i}\right) .{ }^{20}$

\subsection{Identification}

To estimate the causal effect of affiliation on RUC-recommended values, we need variation in affiliation that is uncorrelated with the underlying value of a procedure. Our goal is thus to condition on predicted prices and predicted affiliation, as a function of the RUC membership and the likely specialty proposers for a given procedure, and to exploit the remaining variation. Since prices are relative, we condition on an indicator for the RUC meeting at which a procedure was valued. Further, because both underlying value and likely proposers are most obviously related to the utilization of a procedure by specialties, we condition on 64 specialty utilization shares:

$$
w_{i s}=\frac{\sum_{y} q_{i s y}}{\sum_{y} \sum_{s} q_{i s y}},
$$

for service $i$, specialty $s$, and Medicare claim year $y$.

Figure 3 shows substantial variation in affiliation even across proposals with the participation of a given specialty. A large part of this variation arises from the identities of the proposing specialties when multiple specialties can possibly propose. As many as a dozen specialties are eligible to be on the proposal a typical service, while $98 \%$ of the proposals involve five or fewer specialties. This variation may be uncorrelated with underlying RVU if it relates to variation in a specialty's costs of proposing from meeting to meeting, which in turn depends on the resources

\footnotetext{
${ }^{20}$ In some cases, described below, we will compute the counterfactual set affiliation for proposal $i$ in a different meeting than the actual $t$. In these cases we continue to normalize with the mean and standard deviation of the actual sample of $A\left(\mathcal{R}_{t}, \mathcal{S}_{i}\right)$ in order to maintain comparability.
} 
the specialty has available to administer surveys and send representatives to present a proposal. ${ }^{21}$ Further, in Appendix A-3, we show that with costly proposals and two specialties, there exists no unique pure-strategy equilibrium for proposals, while new mixed-strategy equilibria are introduced. When specialties cannot coordinate, mixing in equilibrium introduces another random source of variation in the identities of proposing specialties.

For identification, we require that proposals with (conditionally) higher affiliation are not for services with (conditionally) higher intrinsic prices. In Table 2, we show balance in characteristics for Medicare beneficiaries who receive services with high residual affiliation and those who receive services with low residual affiliation. In Figure 4, we similarly show balance in predicted price, as a function of these plausibly exogenous service characteristics, linearly controlling for meeting dummies and specialty shares. Despite no relationship with residual affiliation, these characteristics are nonetheless important: They alone explain about $25 \%$ of the variation in prices and are highly correlated with affiliation unconditionally. Further, we consider below two types of violations of our identifying assumption. First, specialties submitting proposals for procedures with intrinsically high prices may choose to submit these proposals at meetings with more affiliated RUC members. Second, holding fixed utilization by specialties, procedures with higher intrinsic prices may also be more likely to have more or particular proposing specialties, which may increase affiliation.

\subsubsection{Endogenous Timing}

We look for evidence that specialties choose to propose when they are more affiliated with the RUC. We compute the affiliation that each proposal $i$ would have over all possible alternative meetings $t^{\prime} \in T \backslash t$, generating a set of counterfactual affiliations, $A=\left\{A\left(\mathcal{R}_{t^{\prime}}, \mathcal{S}_{i}\right)\right\}$. We then test whether observed affiliations are statistically distinguishable from these counterfactual affiliations. As shown in Figure 5, the mean differenced statistic $A\left(\mathcal{R}_{t^{\prime}}, \mathcal{S}_{i}\right)-A\left(\mathcal{R}_{t}, \mathcal{S}_{i}\right)$ over all proposals and possible meeting dates $\left(i, t^{\prime}\right)$ is not statistically different than 0. Further, controlling for average counterfactual affiliation, $\bar{A}\left(\mathcal{S}_{i}\right) \equiv\|T\|^{-1} \sum_{t \in T} A\left(\mathcal{R}_{t}, \mathcal{S}_{i}\right)$, we find no correlation between price predicted from service characteristics and the realized affiliation.

\footnotetext{
${ }^{21}$ For example, we find that a specialty is less likely to propose if there is another procedure in the same RUC meeting that has a higher predicted propensity of the specialty proposing.
} 


\subsubsection{Endogenous Proposing Specialties}

Most of the variation in $A\left(\mathcal{R}_{t}, \mathcal{S}_{i}\right)$ across $i$ and $t$ is due to the proposing specialties, $\mathcal{S}_{i}$, and not to the RUC members, $\mathcal{R}_{t}{ }^{22}$ We thus assess whether specialty participation in proposals, conditional on specialty utilization shares $\mathbf{w}_{i}$, is correlated with the service's predicted price. We first estimate the propensity of each specialty $s$ to propose $i$, as a logit model of $\pi_{i s}=\operatorname{Pr}\left(s \in \mathcal{S}_{i} \mid s, \mathbf{w}_{i}\right)$, which has a pseudo- $R^{2}$ of 0.67 . Conditional on this propensity, actual participation in a proposal is uncorrelated with predicted RVU, despite an adjusted $R^{2}$ of 0.88 for the RVU prediction equation (Figure 6). ${ }^{23}$ This result supports our identifying assumption that, conditional on $\mathbf{w}_{i}$, the remaining variation in specialty-proposal participation is uncorrelated with underlying price. Further, in Appendix A-4, we show that simulated affiliation, based on a richer model of specialty-proposal propensities, $\pi_{i s}$, and $\mathcal{R}_{t}$, exhibits wide variation centered around actual affiliation (Figure A-5). ${ }^{24}$

\section{Affiliation Effect on Prices}

\subsection{Estimated Effect}

We estimate the effect of affiliation on RUC-recommended RVUs with the following equation:

$$
\ln \mathrm{RVU}_{i t}=\alpha A\left(\mathcal{R}_{t}, \mathcal{S}_{i}\right)+\mathbf{X}_{i} \beta+\mathbf{T}_{t} \eta+\mathbf{w}_{i} \zeta+\varepsilon_{i t}
$$

where $\mathrm{RVU}_{i t}$ is the relative price (work RVU) granted to proposal $i$ at meeting $t$, and $\alpha$ is the effect of increasing set affiliation by a standard deviation. ${ }^{25}$ We include fixed effects for the RUC meeting $t$ and control for specialty utilization shares $\mathbf{w}_{i}$. Thus we compare prices within the same meeting and for services with the same (linear) composition of specialties performing the

\footnotetext{
${ }^{22}$ Specifically, we compare $\operatorname{Var}_{i}\left(A\left(\mathcal{R}_{t}, \mathcal{S}_{i}\right)-\bar{A}\left(\mathcal{S}_{i}\right)\right)$ and $\operatorname{Var}_{i}\left(\bar{A}\left(\mathcal{S}_{i}\right)\right)$ relative to $\operatorname{Var}_{i}\left(A\left(\mathcal{R}_{t}, \mathcal{S}_{i}\right)\right)$. The variation due to $t$ is only about $3.7 \%$ of the total variation, implying the variation due to proposing specialty identities is about 27 times greater.

${ }^{23}$ We predict RVU using procedure characteristics, including procedure code word descriptions, surveyed time, prior RVU, and Medicare beneficiary characteristics.

${ }^{24}$ In Appendix A-4, we further provide supporting evidence of wide variation in the propensity of proposing among actual proposers and among the highest-propensity specialties.

${ }^{25}$ We study the effect of affiliation on $\log$ RVU, because relationships between components of price (e.g., time and intensity of a service) are viewed as multiplicative (Hsiao et al., 1988).
} 
service.

We can potentially control for a large number of procedure code and proposal characteristics $\mathbf{X}_{i}$ specific to a service $i$ : (i) prior RVU, which exists for the roughly $50 \%$ of proposals made for existing procedures, (ii) characteristics of Medicare beneficiaries who receive the procedure, (iii) time and work characteristics of the procedure (e.g., total utilization, surveyed time), and (iv) word stems in the procedure's description. ${ }^{26}$

In Table 3, we report results for a variety of control specifications. In the full specification, reported in Column 4, we find that a standard deviation increase in affiliation increases relative price by $10.1 \% \cdot{ }^{27}$ In Figure 7 , we illustrate this result in a binned scatterplot of residualized price on the $y$-axis and residualized affiliation on the $x$-axis. Increasing affiliation from the 10 th percentile to the 90 th percentile would increase prices by $17 \%$.

In Column 5, we show a similar effect when we control for predicted set affiliation, as a function of the RUC membership, $\mathcal{R}_{t}$, and the predicted propensity of each specialty to propose, described in Appendix A-4, instead of linear $\mathbf{w}_{i}$. This prediction mechanically controls for any variation in RUC membership over time. In Column 6, we show that our result is robust to controlling for interactions of each specialty share with linear meeting year, which allows for changes in the average intrinsic value of each specialty's procedures over time. In Table A-3, we show robustness of our results to 49 other formulations of affiliation. ${ }^{28}$ To the extent that we measure affiliation with error, in that we may fail to capture important linkages between specialties (e.g., between anesthesiology and surgery), our results can be interpreted as an lower bound of the effect of affiliation on prices.

\footnotetext{
${ }^{26}$ In practice, because of the high number of procedure code characteristics relative to the number of proposals, we employ methods to avoid overfitting. For example, for a code description's word stems, we remove collinear word stems and then select predictive word stems via LASSO. We also form jack-knifed RVU predictions using the set of post-LASSO OLS controls and using only observations from meetings other than meeting $t$. Finally, we form jack-knifed RVU predictions based on the procedure's characteristics in (iii).

${ }^{27}$ Consistent with robustness across control specifications, in an Altonji et al. (2005) framework we find that selection on unobservables, controlling for meeting dummies and specialty shares, would need to be 3.9 times greater than selection on observables in order to explain our estimated effect.

${ }^{28}$ We defend our preferred affiliation measure and discuss alternatives in Appendix A-2.
} 


\subsection{Counterfactual Revenue}

Given the effect of affiliation on recommended prices, we examine the revenue implications from two counterfactual scenarios that change the affiliation of proposals. In the first scenario, we equalize the affiliation of all proposals, so that no proposal has an advantage (or disadvantage) under affiliation. In the second, we consider a counterfactual RUC, in which the 25 specialty seats are apportioned based on specialty physician populations, as given in Table A-5. This scenario, which generally reallocates RUC seats away from "procedural" specialties, has been a common policy intervention advocated by critics of the RUC who wish to close the "primary care-specialty income gap" (Bodenheimer et al., 2007; Laugesen, 2016).

In both counterfactual scenarios, we hold fixed the timing of each proposal, the Medicare budget, and the utilization of each service over time. We thus simulate changes in revenue at the service level solely through the effect of counterfactual affiliation on service prices. We further aggregate counterfactual revenue reallocation to specialties and to types of services, defined by Berenson-Eggers Type of Service (BETOS) codes. We provide details of the simulation algorithm in Appendix A-5.

Equalizing affiliation across proposals would reallocate $\$ 1.0$ billion (or $2.9 \%$ of work-based reimbursement) in yearly Medicare work-based revenue across procedures, or $\$ 1.9$ billion in total Medicare reimbursement, if we extend the affiliation effect to practice-expense reimbursement (also priced by the RUC). Assuming a proportional price change in private insurance, the crossservice reallocation would be $\$ 13.4$ billion yearly. We also evaluate which specialties and types of procedures, grouped by BETOS categories, would see the largest revenue reallocations. We illustrate those changes in Figure 8. Although internal medicine has a minority of seats, internal medicine gains from affiliation because many other specialties, including surgical ones, also derive a large share of revenue from the same evaluation and management services performed in office and inpatient visits. ${ }^{29}$ Of specialties, emergency medicine would have the largest percentage revenue gain $(+17 \%)$, while infectious disease would have the largest loss $(-5.8 \%)$. Overall, $1.9 \%$ of revenues would be reallocated across specialties, about $\$ 1.3$ billion in Medicare spending

\footnotetext{
${ }^{29}$ We do not investigate other mechanisms, such as the difficulty in raising prices for common procedures, that may depress prices for office visits and therefore affect the revenues of non-procedural specialties (Bodenheimer et al., 2007).
} 
or $\$ 8.9$ billion in annual health care spending from Medicare and private insurance.

Reapportioning RUC seats based on specialties' relative physician populations would reallocate $\$ 230$ million in yearly Medicare work-based revenue across procedures, or $\$ 450$ million in total Medicare reimbursement. Overall, reallocation figures generally are only one-fifth of the magnitude (and often opposite in direction) of the reallocation when equalizing affiliation. Even though internal medicine would be given 4 seats, compared to the actual average of 1.5 seats on the RUC, the specialty would gain less than 1 percent in revenue. Infectious disease would have the largest percentage revenue gain $(+1.4 \%)$, and ophthalmology would experience the largest percentage revenue loss $(-1.4 \%)$. We illustrate other individual specialty and BETOS code reallocations in Figure 9.

\subsection{Interpreting the Price Effect as Bias}

We interpret the finding that greater affiliation results in higher prices as evidence of a bias among RUC members to recommend higher prices for affiliated specialties. This interpretation is consistent with a recent empirical literature on political rents. ${ }^{30}$ However, preferences typically correlate with the quality of information decision-makers hold. Could the higher prices we observe be due to better ex ante information held by affiliated RUC members rather than bias? ${ }^{31}$ We argue that this alternative is less plausible.

First, we observe that if the RUC were unbiased but held different ex ante levels of information across different decisions, then its price recommendations should not vary on average in a Bayesian equilibrium. For example, if the RUC were to systematically underestimate prices when uninformed, it would benefit from adjusting its recommendations upward by the average level of its underestimate. This feature holds true in standard models of decision-making under asymmetric information. ${ }^{32}$ More recent models allow for information rents to be held by an

\footnotetext{
${ }^{30}$ For notable examples in the economics literature, see Fisman (2001); Khwaja and Mian (2005); Faccio (2006); Ferguson and Voth (2008). This literature generally views relationships between firm valuations and political actors as prima facie evidence of rents and corruption. In medical price-setting, Bertoli and Grembi (2017) study regional-government inpatient prices for obstetric admissions in Italy, as a function of the number of physicians in government positions. Recent papers of committee decision-making, by Li (2017) and Camara and Kyle (2017), explicitly consider information alongside bias. Their frameworks would also interpret decisions systematically skewed toward or against randomly assigned applicants (i.e., equal expected quality) as bias.

${ }^{31}$ It is important to distinguish ex ante information held by the RUC, before a proposal, from information that is extracted in the proposal process. The latter is the focus of Section 5.

${ }^{32}$ This intuition is most straightforward when decisions are continuous. In the RUC context, we can think
} 
asymmetrically informed sender, e.g., when the sender's message cannot be fully expropriated to the receiver's advantage (Callander, 2008). ${ }^{33}$ Second, and more importantly, because the ex ante information story relies on the RUC possessing greater information for procedures with higher prices, we would need changes in RUC membership to observe changes in information. In our empirical setting, however, the main source of identifying variation comes from the identities of the proposing specialties, not changes to the RUC's composition. Our use of proposer variation relates to a literature on lobbying, in which lobbyists (proposers) and may lead to favorable decisions by their very identity (Blanes i Vidal et al., 2012; Bertrand et al., 2014) and the alignment of their interests with those of their audience (Kessler and Krehbiel, 1996; Hirsch and Montagnes, 2015). ${ }^{34}$

In Appendix A-6 and Table A-4, we empirically investigate alternative mechanisms behind the price effect. Specifically, we consider signaling "buy-in," in which services with higher underlying prices should have more proposers (and therefore higher affiliation). We also consider whether the RUC recommendations for a service are explained by service-specific revenue or utilization by RUC member specialties. We show that the effect of affiliation on prices is robust to controlling for number of proposers and measures of service-specific interests (or information) held by RUC specialties.

of decisions as continuous in the following way: RUC members often publicly voice their view of "reasonable" prices; members discuss and renegotiate rejected prices in "facilitation committees" (see Section 2.2); and the RUC may unilaterally recommend a price to the government if no reasonable proposal is made. Even in a discrete context, the same intuition applies. With a fully informed RUC, proposed prices would always equal the true price. An uninformed RUC will have a threshold price above which it chooses to reject a price recommendation. In equilibrium, all proposals should then be at this price. Therefore, the Bayesian equilibrium RUC threshold will equal the RUC's prior, which equals the expectation of the true price.

${ }^{33}$ The view that asymmetric information benefits experts is intuitive. Without this asymmetry, experts would have no incentives to acquire knowledge. On page 232 of Weber (2009): "Under normal conditions, the power position of a fully developed bureaucracy is always overtowering. The 'political master' finds himself in the position of the 'dilettante' who stands opposite the 'expert."' This Weberian view of expertise is well-known in political science and sociology: for example, Bendor et al. (1985, p. 1042) notes, "A bureau's influence rests not on its formal authority - its ability to manipulate the rules-but as Weber noted, its control of information, its ability to manipulate ... information about the consequences of different alternatives."

${ }^{34}$ In Appendix A-4, we present regression specifications to test two alternative mechanisms behind the affiliation result. First, we examine a signaling hypothesis in which affiliation simply reflects the intrinsic value of a procedure, and the unbiased RUC reacts to that signal. Second, we look for informational mechanisms at the procedure level by testing whether the affiliation effect is driven by procedures performed often by specialties with seats on the RUC. We find little evidence that either of these mechanisms explains our main affiliation effect. 


\section{$5 \quad$ Affiliation Effect on Information Extraction}

Given the evidence of bias due to affiliation, we return to a broader question posed by the prevalence of advisory committees: Why would the government involve an intermediary that may be biased toward industry? In this section, we first introduce a conceptual model that illustrates a trade-off between bias and information extraction. In our framework, the specialty society is a biased expert who has information about the true value of a service to be priced. We show that the quality of information extracted and used in price-setting may improve with affiliation between the RUC and the specialty society. We then test the predictions of this model using two unique and objective measures of information quality. First, we test for the effect of greater affiliation on the quality of survey information presented to the RUC. Second, we use data on prices from private insurers to evaluate how price-following from Medicare to the private sector depends on affiliation.

\subsection{Conceptual Framework}

Consider a government that procures a service at price $p$, ideally set at $\theta \sim U(0,1)$. A specialty society knows $\theta$ but may also have bias. The government may delegate price-setting to the RUC, which then evaluates information from the specialty about $\theta .{ }^{35}$ Information can be communicated in two forms: "hard" and "soft." Hard information is verifiable and interpretable but costly to produce. In this setting, hard information includes the data reported in physician surveys, for example. Soft information, as in "cheap talk" (Crawford and Sobel, 1982), includes aspects of the service that cannot be verified by evidence, such as the "difficulty" or "complexity" of one service relative to another.

The government chooses the specialty composition of the RUC, so that the RUC may be more or less affiliated with the proposer. The degree of bias in price-setting and the quality of information will depend on this affiliation between the RUC and the speciality society.

\footnotetext{
${ }^{35}$ We follow a standard setup from Dessein (2002). This modeling assumption may be supported by the fact that Medicare follows the RUC price recommendations $90 \%$ of the time. More recent cheap talk models study sequential cheap talk and are more complicated. If the government undoes bias from high-affiliation RUC decisions, then informational advantages from communication will in general be nullified (Ambrus et al., 2013).
} 


\subsubsection{Timing and Payoffs}

The timing and payoffs are as follows:

1. The government delegates to a RUC intermediary with bias $b_{R}$.

2. The specialty may produce hard information verifying that $\theta$ uniformly lies on a subinterval of length $L$ (i.e., $\theta \sim U(\underline{\theta}, \bar{\theta}), L \equiv \bar{\theta}-\underline{\theta} \in[0,1]$ ), via a technology that comes at cost $c(L) \cdot{ }^{36} c(1)=0, c^{\prime}(L)<0$, and $c^{\prime \prime}(L)>0$.

3. The specialty observes $\theta$, and then transmits a cheap talk message $m$ about $\theta$.

4. The RUC sets price $p$. Non-transferrable payoffs are as follows for the specialty $\left(u_{S}\right)$, RUC $\left(u_{R}\right)$, and the government $\left(u_{G}\right)$ :

$$
\begin{aligned}
& u_{S}=-\left(\theta+b_{S}-p\right)^{2}-c(L) \\
& u_{R}=-\left(\theta+b_{R}-p\right)^{2} \\
& u_{G}=-(\theta-p)^{2}
\end{aligned}
$$

where $b_{S}$ and $b_{R}$ are biased preferences for the specialty and RUC, respectively, and $b_{S}>0$ without loss of generality.

As in the standard cheap talk model, bias $b_{S}$ and $b_{R}$ enter the specialty and RUC utilities, respectively, such that even though these agents may prefer higher or lower prices than the government, neither prefers to raise or lower prices without bound. ${ }^{37}$

\subsubsection{Comparative Statics}

We consider the comparative statics of changing the RUC's bias, $b_{R}$, with details in Appendix A-7. First, if all information is soft (i.e., $L=1$ regardless), outcomes follow Dessein (2002): If

\footnotetext{
${ }^{36}$ In this exposition, we treat $\bar{\theta}-\underline{\theta}$ as known and assert that $\theta \sim U(\underline{\theta}, \bar{\theta})$. However, this is not technically correct for all values of $L$. In Appendix A-7.4, we consider $\bar{\theta}-\underline{\theta}$ as random, i.e., $L=E[\bar{\theta}-\underline{\theta}]$, which allows $\theta$ to remain uniformly distributed in the posterior interval. Neither the uniform distribution of $\theta$ nor fixed $\bar{\theta}-\underline{\theta}$ is required for the intuition of this model.

${ }^{37}$ This can be interpreted as a common preference held by all agents for "sensible" prices that are neither too high nor too low; they may directly value this sensibility or they may value credibility to the government to ensure they continue to have a role in setting prices.
} 
the government chooses a RUC with preferences biased toward the specialty (i.e., $b_{R}$ close to $\left.b_{S}\right)$, the expected price will move away from the government's ideal, but more information is communicated. The optimal RUC bias is $b_{R}^{*} \in\left[0, b_{S}\right]$. If $b_{S}$ is sufficiently large, then $b_{R}^{*}=0$; if $b_{S}$ is sufficiently small, then $b_{R}^{*}=b_{S}$. But it is never optimal to have $b_{R}^{*}<0$ or $b_{R}^{*}>b_{S}$, because this worsens both bias and communication.

Second, when we allow the specialty to produce hard information-reducing the space $[\underline{\theta}, \bar{\theta}]$ to length $L<1$ with verifiable evidence- such evidence eliminates the need to communicate a service's value through soft channels. Hard information is most valuable when soft communication is least feasible, such as in settings in which the RUC and specialty proposer have divergent preferences. This implies that greater $b=b_{S}-b_{R}$ (i.e., low affiliation between the RUC and the specialty) induces the specialty to produce more hard information. Since hard information increases the government's utility, the optimal RUC has preferences closer to the government's $\left(b_{R}^{*}\right.$ is closer to 0$)$ when hard information is easier to produce. As the technology to produce hard information improves (i.e., $c(L)$ becomes smaller), the optimal $b_{R}^{*}$ moves closer to $0 .{ }^{38}$

In summary, our model predicts that higher affiliation will allow better communication of soft information between proposers and the RUC. Hard information provision, by contrast, decreases with affiliation. Thus, the overall information content of prices as a function of affiliation depends on how much each type of information adjusts. When the cost (or feasibility) of producing hard information falls, the degree of affiliation that maximizes information extraction will decrease. We next test these comparative statics using our empirical measures of information quality.

\subsection{Affiliation Effect on Hard Information}

Unlike many other settings, our dataset contains an objective measure of hard information. As we describe in Section 2, when specialties propose a new RVU, they present survey evidence about the work involved in delivering a service, particularly the time needed (Zuckerman et al., 2016; Burgette et al., 2016). We use this survey data as our measure of hard information - the more physicians that a specialty or a coalition of specialties surveys about physician work, the

\footnotetext{
${ }^{38}$ In Appendix A-7, we show that it is never optimal to have $b_{R}<0$. In Figure A-6, we illustrate this relationship between welfare (government expected utility) and $b_{R}$, letting the cost of hard information, $c(L)$, vary.
} 
more concrete is the evidence presented in a proposal to the RUC ${ }^{39}$ However, surveying more physicians is costlier to specialty societies.

Using per-specialty survey sample size and the number of respondents as measures of hard information, denoted $H_{i t}$, we estimate the affiliation effect on hard information measure with the following regression: ${ }^{40}$

$$
\ln H_{i t}=\alpha A\left(\mathcal{R}_{t}, \mathcal{S}_{i}\right)+\mathbf{X}_{i} \beta+\mathbf{T}_{t} \eta+\mathbf{w}_{i} \zeta+\varepsilon_{i t},
$$

We use the same controls as in Equation (6). The coefficient of interest, $\alpha$, reflects the effect of affiliation on the endogenous decision to provide hard information. The number of specialties on a proposal may also affect survey samples, e.g., through coordination issues. Therefore, to isolate empirically the mechanism of affiliation on hard information, we may also control for indicators of the number of specialty proposers.

We present results in Table 4. We see strong negative effects: In our preferred specification, controlling for proposer utilization of the a procedure, in Column 2, a one standard-deviation increase in affiliation decreases per-specialty survey sample size by $33.2 \%$ and per-specialty number of respondents by $41.3 \%$. Figure 10 shows these results in a binned scatterplot of residual log survey counts against residual set affiliation. The negative effect persists when controlling for the number of specialty proposers, shown in Column 4 of Table 4, although the effect is not statistically significant for the outcome of survey respondents.

\subsection{Price Transmission to Private Insurance}

As a second measure of information quality, we examine how private prices track changes in Medicare prices, depending on the source of the Medicare price and the affiliation of the proposal that led to a given RUC-recommended price. Recent research documents strong price-following

\footnotetext{
${ }^{39}$ The RUC often focuses on the distribution of survey outcomes and the number of survey respondents, as a marker of the credibility of a proposal. Although any given survey respondent may exaggerate his or her response, it is more difficult to do so (and generally more costly to lie) in aggregate when there are many respondents, along the lines of (Kartik, 2009).

${ }^{40}$ While the total surveyed information is obviously relevant from the perspective of the RUC, there are mechanical rules that require specialties to survey a minimum number of physicians, conditional on surveying (American Medical Association, 2017). Therefore, for proposals with more than one specialty, we consider the effect of affiliation on per-specialty hard information.
} 
from Medicare to private insurance prices (Clemens and Gottlieb, 2017; Clemens et al., 2017). This literature notes two potential mechanisms behind price-following: Medicare may serve as an outside option in bargaining between private insurers and physicians, or Medicare may provide a "knowledge standard" with information content.

We focus on the latter mechanism and test the overall informational content of Medicare prices. If Medicare price changes serve solely as a bargaining benchmark, then the degree to which they are followed should not depend on their source and, in particular, on the affiliation of a proposal at the time of the RUC's vote. Private insurers may choose to respond differentially to Medicare price changes if they believe some changes result from a more informed committee, or, alternatively, if their own internal due-diligence agrees with the RUC's assessment. ${ }^{41}$ Secondarily, we assess whether Medicare price changes from higher-affiliation proposals are also discounted uniformly by private insurance, suggesting private insurers may adjust to possible bias.

We first construct private and Medicare prices by dividing total charges by total number of claims observed in MarketScan and Medicare data for a given procedure code in a given year. To allow for lagged price transmission to private insurance, we normalize log prices within payer and then match private prices for each code $i$ and year $y$ to a Medicare price for the same code in the year $y^{M}(i, y) \in\{y, y-1, y-2\} .{ }^{42}$ We then estimate variants of the following regression to assess average price transmission:

$$
\ln \operatorname{Price}_{i, y}^{P}=\beta \ln \operatorname{Price}_{i, y^{M}(i, y)}^{M}+\mathbf{T}_{i y} \eta+\xi_{i}+\varepsilon_{i y},
$$

where $\mathbf{T}_{i y}$ is a vector of time dummies (year $y$, Medicare year $y^{M}$, and the RUC meeting, for Medicare prices associated with a RUC decision) and $\xi_{i}$ is a service fixed effect for the procedure

\footnotetext{
${ }^{41}$ In interviews with RUC members, one described an informal process in which private insurance administrators consult with trusted clinical sources (often friends) who perform procedures, asking whether prices seemed reasonable.

${ }^{42}$ In detail, we normalize log prices to have a frequency-weighted mean of 0 within payer (private or Medicare) and year, and we then match private prices for each code $i$ and year $y$ to a Medicare price for the same code in the year $y^{M}(i, y) \in\{y, y-1, y-2\}$ with the closest log price change:

$$
y^{M}(i, y)=\underset{y^{\prime} \in\{y, y-1, y-2\}}{\arg \min }\left|\Delta \ln \operatorname{Price}_{i, y}^{P}-\Delta \ln \operatorname{Price}_{i, y^{\prime}}^{M}\right| .
$$

$\Delta \ln \operatorname{Price}_{i, y}^{P} \equiv \ln \operatorname{Price}_{i, y}^{P}-\ln$ Price $_{i, y-1}^{P}$ is a change in the normalized log private prices for service $i$ in year $y$, and $\Delta \ln$ Price $_{i, y}^{M}$ is the analogous Medicare log price change.
} 
code. The service fixed effect implies that we focus on changes in private insurance prices in response to changes in Medicare prices, holding constant any characteristic of the service. We also estimate pooled regressions across categories of Medicare prices:

$$
\ln \operatorname{Price}_{i y}^{P}=\sum_{C}\left(\alpha_{C}+\beta_{C} \ln \operatorname{Price}_{i, y^{M}(i, y)}^{M}\right) \cdot \mathbf{1}(C(i, y)=C)+\mathbf{T}_{i y} \eta+\xi_{i}+\varepsilon_{i y}
$$

where $C$ references one of three categories of Medicare price by source: (i) Medicare prices for a code in a year with an associated RUC decision and an above-median proposal affiliation, (ii) prices with a RUC decision and a below-median proposal affiliation, and (iii) prices not associated with a RUC decision. Most Medicare prices fall in the last category, but, as shown in Figure A-7, prices changes in this category are smaller. ${ }^{43}$ To facilitate closer comparison of the "non-RUC" and "RUC" Medicare prices in the pooled regressions, we restrict attention to non-RUC log price changes of at least 0.3 in absolute value.

In Table 5, our estimates suggest that private prices follow RUC-based Medicare prices to a much larger extent than non-RUC Medicare prices. Estimating Equation (8) separately for RUC and non-RUC Medicare prices and including service fixed effects shows this result most starkly. Within procedure code, log price changes in Medicare originating from the RUC are transmitted to private insurance with a coefficient of 0.892 (Column 1), while those that have no associated RUC recommendation are transmitted with a coefficient of 0.399 (Column 2) or 0.300 (Column 3), depending on whether the sample includes all non-RUC changes or is restricted to larger changes. Further RUC-based Medicare prices originating from high-affiliation proposals show slightly higher following than those from low-affiliation proposals. ${ }^{44}$

Figure 11 shows pooled results, both without and with service fixed effects, corresponding to Columns 4 and 5 of Table 5. The figure reproduces differences in the slopes of the lines tracing private prices to Medicare prices that depend on the source of the Medicare price. This suggests

\footnotetext{
${ }^{43}$ Medicare average price changes with no associated RUC recommendation in our dataset may occur for a variety of reasons, including changes in the geographic composition of claims, changes in the facility vs. nonfacility composition of claims, conversion factor adjustments, and changes in the practice expense component of RVUs alone.

${ }^{44}$ We also analyze this question in a specification with private log price changes regressed on Medicare log price changes and find similar results. As shown in Figure A-8, high-affiliation RUC price changes result in steeper private price changes than low-affiliation RUC price changes.
} 
that Medicare price changes that originate from RUC decisions, and in particular from highaffiliation RUC decisions, appear more informative for private insurance. In addition to steeper slopes, the lines are generally lower in levels for RUC Medicare prices (and further for those from high-affiliation proposals). These uniformly lower private insurance price changes suggest that private insurance may, to an extent, reverse the bias induced by affiliation. ${ }^{45}$

\section{Conclusion}

We find evidence of bias or regulatory capture in Medicare's price setting process. Increasing affiliation between the specialties proposing a value and the RUC from the 10th to the 90th percentile would result in a $17 \%$ higher relative price recommendation. However, we also find that involving a committee of physicians in setting prices can improve the quality of information used in the process. We find patterns suggesting that private insurers follow Medicare prices more closely when the public prices originate from a RUC vote. The private sector appears to adjust downward in levels for bias in the RUC's recommendations, but nonetheless reacts more strongly in terms of slope to price changes from more highly affiliated RUC votes.

Our findings suggest Medicare faces a balancing act in setting prices. Inviting input from the RUC may introduce bias in prices, but it may also improve the information extracted from specialties. We show in counterfactuals how undoing this bias, either by equalizing affiliation or by changing the RUC's membership, reallocates revenue across specialties and creates winners and losers within medicine. These analyses, however, ignore likely quantity effects from price changes, which generate real welfare effects beyond transfers in revenue. To the extent physicians are imperfect agents for their patients and deviate toward procedures with greater reimbursement levels (Clemens and Gottlieb, 2014; Gruber et al., 1999), and if physicians-in-training avoid specializing in fields with lower procedural income, the actions of the RUC may have broader welfare consequences for health care.

\footnotetext{
${ }^{45}$ In Appendix A-8, we consider alternatives to our interpretation that affiliation facilitates better information through communication. First, the RUC may have more information on high-affiliation decisions, even without communication, because its members are more likely to perform the services in question. Second, Medicare and private insurance are more likely to get the price "right" for high-volume procedures, which are also more likely to have RUC decisions and high-affiliation proposals. Third, there may be some other unspecified predictor of price transmission that could be correlated with affiliation. We find that our results are robust, accounting for these potential alternative mechanisms.
} 


\section{References}

Aggarwal, C. C., A. Hinneburg, and D. A. Keim (2001): "On the surprising behavior of distance metrics in high dimensional spaces," in ICDT, Springer, vol. 1, 420-434.

Aghion, P. And J. Tirole (1997): "Formal and real authority in organizations," The Journal of Political Economy, 105, 1-29.

Altonji, J. G., T. E. Elder, and C. R. Taber (2005): "Selection on Observed and Unobserved Variables: Assessing the Effectiveness of Catholic Schools," Journal of Political Economy, 113, 151-184.

Ambrus, A., E. Azevedo, And Y. Kamada (2013): "Hierarchical cheap talk," Theoretical Economics, 8, 233-261.

American Medical Association (2014): AMA/Specialty Society RVS Update Process, Chicago: AMA.

— (2015): The Physicians' Guide: Medicare RBRVS, Chicago: AMA.

— (2017): AMA/Specialty Society RVS Update Process, Chicago: AMA.

Association of American Medical Colleges (2016): "Physician Specialty Data Report," Tech. rep., Washington, DC.

Austen-Smith, D. (1994): "Strategic Transmission of Costly Information," Econometrica, 62, 955.

Bendor, J., S. TAYlor, And R. V. GaAlen (1985): "Bureaucratic Expertise versus Legislative Authority: A Model of Deception and Monitoring in Budgeting," American Political Science Review, 79, 1041-1060.

Berenson, R. A. And J. D. Goodson (2016): "Finding Value in Unexpected Places: Fixing the Medicare Physician Fee Schedule," New England Journal of Medicine, 374, 1306-1309.

Bertoli, P. And V. Grembi (2017): "The political economy of diagnosis-related groups," Social Science \& Medicine, 190, 38-47.

Bertrand, M., M. Bombardini, And F. Trebbi (2014): "Is It Whom You Know or What You Know? An Empirical Assessment of the Lobbying Process," American Economic Review, 104, 3885-3920.

Blanes i Vidal, J., M. Draca, And C. Fons-Rosen (2012): "Revolving Door Lobbyists," American Economic Review, 102, 3731-3748.

Bloom, N., M. Schankerman, and J. Van Reenen (2013): "Identifying Technology Spillovers and Product Market Rivalry," Econometrica, 81, 1347-1393. 
Bodenheimer, T., R. A. Berenson, and P. Rudolf (2007): "The Primary Care-Specialty Income Gap: Why It Matters," Annals of Internal Medicine, 146, 301-306.

Braeutigam, R. R. And J. C. Panzar (1993): "Effects of the Change from Rate-of-Return to Price-Cap Regulation," The American Economic Review, 83, 191-198.

Brown, M. B. (2009): "Federal advisory committees in the United States: A survey of the political and administrative landscape," in Scientific Advice to Policy Making: International Comparison, Opladen, Germany, and Farmington Hills, MI: Verlag Barbara Budrich., 17-39.

Burgette, L. F., A. W. Mulcahy, A. Mehrotra, T. Ruder, and B. O. Wynn (2016): "Estimating Surgical Procedure Times Using Anesthesia Billing Data and Operating Room Records," Health Services Research.

Caillaud, B. And J. Tirole (2007): "Consensus Building: How to Persuade a Group," American Economic Review, 97, 1877-1900.

Callander, S. (2008): "A Theory of Policy Expertise," Quarterly Journal of Political Science, 3, 123-140.

CAlvert, R. L. (1985): "The Value of Biased Information: A Rational Choice Model of Political Advice," The Journal of Politics, 47, 530-555.

Camara, F. And M. Kyle (2017): "Experts and Financial Ties: Evidence from FDA Advisory Committees," Tech. rep., University of Southern California.

Centers for Medicare and Medicaid Services (2014): "Estimated Sustainable Growth Rate and Conversion Factor, for Medicare Payments to Physicians in 2015," Tech. rep.

Clemens, J. And J. D. Gottlieb (2017): "In the Shadow of a Giant: Medicare's Influence on Private Physician Payments," Journal of Political Economy, 125, 1-39.

Clemens, J., J. D. Gottlieb, And T. L. Molnar (2017): "Do health insurers innovate? Evidence from the anatomy of physician payments," Journal of Health Economics, Forthcoming.

Clemens, J. P. And J. D. Gottlieb (2014): "Do Physicians' Financial Incentives Affect Medical Treatment and Patient Health?" American Economic Review, 104, 1320-1349.

Clough, J. D. And M. MCClellan (2016): "Implementing MACRA: Implications for Physicians and for Physician Leadership," JAMA, 315, 2397.

Crawford, V. P. And J. Sobel (1982): "Strategic information transmission," Econometrica, 50, 1431-1451.

Cutler, D., E. Glaeser, And J. Vigdor (1999): "The Rise and Decline of the American Ghetto," Journal of Political Economy, 107, 455-506. 
Dessein, W. (2002): "Authority and Communication in Organizations," Review of Economic Studies, 69, 811-838.

Dewatripont, M. And J. Tirole (1999): “Advocates," Journal of Political Economy, 107, $1-39$.

Esteban, J., L. Mayoral, And D. Ray (2012): "Ethnicity and Conflict: An Empirical Study," The American Economic Review, 102, 1310-1342.

Faccio, M. (2006): "Politically Connected Firms," American Economic Review, 96, 369-386.

FAnG, H. AND Q. Gong (2017): "Detecting Potential Overbilling in Medicare Reimbursement via Hours Worked," American Economic Review, 107, 562-591.

Ferguson, T. And H.-J. Voth (2008): "Betting on Hitler: The Value of Political Connections in Nazi Germany," Quarterly Journal of Economics, 123, 101-137.

Fisman, R. (2001): "Estimating the Value of Political Connections," American Economic Review, 91, 1095-1102.

Gentzkow, M. And J. M. Shapiro (2011): "Ideological Segregation Online and Offline," The Quarterly Journal of Economics, 126, 1799-1839.

Grossman, G. M. and E. Helpman (2001): Special interest politics, MIT press.

Gruber, J., J. Kim, And D. Mayzlin (1999): "Physician fees and procedure intensity: the case of cesarean delivery," Journal of Health Economics, 18, 473-490.

Hirsch, A. V. And B. P. Montagnes (2015): "The Lobbyist's Dilemma: Gatekeeping and the Profit Motive," Tech. rep., California Institute of Technology.

Hirsch, A. V. And K. W. Shotts (2015): "Competitive Policy Development," American Economic Review, 105, 1646-64.

Ho, K. And R. S. LeE (2017): "Insurer Competition in Health Care Markets," Econometrica, $85,379-417$.

Hsiao, W. C., P. Braun, D. Dunn, and E. R. Becker (1988): "Resource-based relative values. An overview," JAMA, 260, 2347-2353.

JAffe, A. B. (1986): "Technological Opportunity and Spillovers of R \& D: Evidence from Firms' Patents, Profits, and Market Value," The American Economic Review, 76, 984-1001.

Kartik, N. (2009): "Strategic Communication with Lying Costs," The Review of Economic Studies, 76, 1359-1395.

Kessler, D. And K. Krehbiel (1996): "Dynamics of Cosponsorship," The American Political Science Review, 90, 555. 
Khwaja, A. I. And A. Mian (2005): "Do Lenders Favor Politically Connected Firms? Rent Provision in an Emerging Financial Market," The Quarterly Journal of Economics, 120, 13711411.

Krishna, V. And J. Morgan (2001): "A Model of Expertise," The Quarterly Journal of Economics, 116, 747-775.

Laugesen, M. J. (2016): Fixing Medical Prices, Harvard University Press.

Laugesen, M. J., R. Wada, And E. M. Chen (2012): "In Setting Doctors' Medicare Fees, CMS Almost Always Accepts The Relative Value Update Panel's Advice On Work Values," Health Affairs, 31, 965-972.

Lewis, M. S. And K. E. Pflum (2015): "Diagnosing Hospital System Bargaining Power in Managed Care Networks," American Economic Journal: Economic Policy, 7, 243-274.

LI, D. (2017): "Expertise versus Bias in Evaluation: Evidence from the NIH," American Economic Journal: Applied Economics, 9, 60-92.

Li, H., S. Rosen, And W. Suen (2001): "Conflicts and Common Interests in Committees," American Economic Review, 91, 1478-1497.

Nicholson, S. And N. Souleles (2001): "Physician Income Expectations and Specialty Choice," Tech. Rep. w8536, National Bureau of Economic Research, Cambridge, MA.

PEAR, R. (2015): "Federal Investigators Fault Medicare's Reliance on Doctors for Pay Standards," The New York Times.

Peltzman, S. (1976): "Toward a More General Theory of Regulation," The Journal of Law and Economics, 19, 211-240.

Potters, J. And F. Van Winden (1992): "Lobbying and Asymmetric Information," Public Choice, 74, 269-292.

Sinsky, C. A. And D. C. Dugdale (2013): "Medicare Payment for Cognitive vs Procedural Care: Minding the Gap," JAMA Internal Medicine.

Stigler, G. J. (1971): "The Theory of Economic Regulation," The Bell Journal of Economics and Management Science, 2, 3.

Weber, M. (2009): From Max Weber: Essays in Sociology, Routledge, google-Books-ID: IIb69vVaQRUC.

White, M. J. (1986): "Segregation and Diversity Measures in Population Distribution," Population Index, 52, 198. 
Whoriskey, P. And D. Keating (2013): "How a secretive panel uses data that distorts doctors' pay," Washington Post.

Wynn, B. O., L. F. Burgette, A. W. Mulcahy, E. N. Okeke, I. Brantley, N. Iyer, T. Ruder, And A. Mehrotra (2015): "Development of a Model for the Validation of Work Relative Value Units for the Medicare Physician Fee Schedule," Research Report, RAND Corporation.

Zinovyeva, N. And M. Bagues (2015): "The Role of Connections in Academic Promotions," American Economic Journal: Applied Economics, 7, 264-292.

Zuckerman, S., K. Merrell, R. A. Berenson, S. Mitchell, D. Upadhyay, And R. Lewis (2016): "Collecting Empirical Physician Time Data: Piloting an Approach for Validating Work Relative Value Units," Tech. rep., Urban Institute. 
Table 1: Specialty Seats on the RUC

\begin{tabular}{lclc} 
Specialty & Meetings & Specialty & Meetings \\
\hline Anesthesiology & 63 & Oncology & 12 \\
Cardiology & 63 & Ophthalmology & 63 \\
Child Psychiatry & 6 & Orthopedic Surgery & 63 \\
Colorectal Surgery & 6 & Otolaryngology & 63 \\
Dermatology & 63 & Pathology & 63 \\
Emergency Medicine & 63 & Pediatric Surgery & 12 \\
Family Medicine & 63 & Pediatrics & 63 \\
Gastroenterology & 20 & Plastic Surgery & 63 \\
General Surgery & 63 & Psychiatry & 63 \\
Geriatrics & 30 & Pulmonary Medicine & 18 \\
Infectious Disease & 9 & Radiation Oncology & 5 \\
Internal Medicine & 63 & Radiology & 63 \\
Nephrology & 6 & Rheumatology & 17 \\
Neurology & 50 & Spine Surgery & 6 \\
Neurosurgery & 63 & Thoracic Surgery & 63 \\
Nuclear Medicine & 7 & Urology & 63 \\
Obstetrics and Gynecology & 53 & Vascular Surgery & 18 \\
\hline
\end{tabular}

Note: This table shows the numbers meetings during which a specialty had a member on the RUC from May 1992 to April 2013. There were a total of 63 meetings during this time period. Each year generally had three meetings, except for the years 1992, 2001, and 2013, which each had two meetings. There were officially four meetings in 1993, but we considered the April and June meetings as one meeting. Each of the specialties listed had one seat at each of its meetings, except for internal medicine, which had two seats in 25 meetings. In our analysis, we considered child psychiatry as psychiatry, since there is no specialty code for child psychiatry in the Medicare data. Similarly, we considered nuclear medicine as radiology. Three meetings had either no services reviewed or had no observations remaining after the sample selection procedure described in Table A-1. Finally, the American Medical Association, the American Osteopathic Association, and Health Care Professional Advisory Committee (HCPAC) each had a permanent voting seat throughout this time period; we did not include them in our analysis. 
Table 2: Balance in Medicare Beneficiary Characteristics

\begin{tabular}{lccc}
\hline Medicare Beneficiary & $\begin{array}{c}\text { Affiliation } \\
\text { above mean }\end{array}$ & $\begin{array}{c}\text { Affiliation } \\
\text { below mean }\end{array}$ & $p$-value \\
\hline Male & 0.471 & 0.470 & 0.371 \\
& $(0.107)$ & $(0.101)$ & \\
Rural & 0.794 & 0.792 & 0.784 \\
Age $>75$ & $(0.052)$ & $(0.054)$ & \\
& 0.405 & 0.416 & 0.366 \\
Age $>$ 85 & $(0.109)$ & $(0.106)$ & \\
Medicare aged & 0.131 & 0.135 & 0.745 \\
Medicare disabled & $(0.067)$ & $(0.067)$ & \\
Medicare ESRD & 0.767 & 0.782 & 0.463 \\
White race & $(0.126)$ & $(0.108)$ & \\
Black race & 0.155 & 0.147 & 0.426 \\
& $(0.062)$ & $(0.058)$ & \\
Hispanic race & 0.063 & 0.054 & 0.903 \\
Other race & $(0.114)$ & $(0.079)$ & \\
& 0.828 & 0.837 & 0.148 \\
& $(0.077)$ & $(0.074)$ & \\
& 0.111 & 0.105 & 0.989 \\
& $(0.059)$ & $(0.052)$ & \\
& 0.025 & 0.024 & 0.109 \\
& $(0.012)$ & $(0.013)$ & \multirow{2}{*}{0.018} \\
\hline
\end{tabular}

Note: This table shows average Medicare beneficiary characteristics for procedure codes in proposals with above- versus below-mean affiliation. We residualize each characteristic, controlling for meeting identities and specialty shares $\mathbf{w}_{i}$. In each cell, we present averages of this residual, conditional on either above- or below-mean affiliation, adding back the unconditional mean to aid in interpretation. Standard deviations of each residualized characteristic are given in parentheses. The last column lists the $p$-value for the null hypothesis that the average residual characteristic is not significantly different between samples corresponding to above- and below-mean affiliation. 


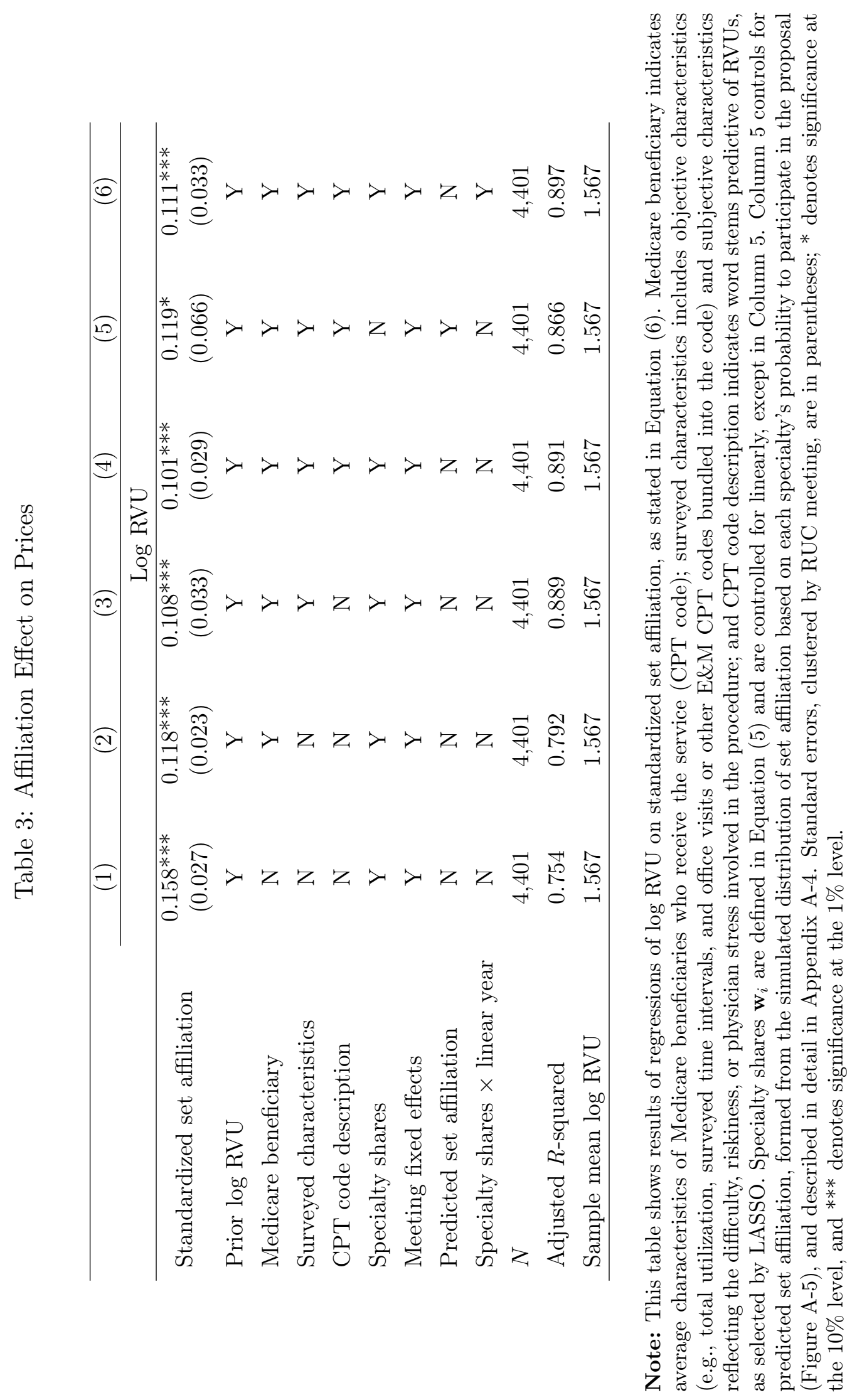


Table 4: Affiliation Effect on Hard Information

\begin{tabular}{lccc}
\hline & $(1)$ & $(2)$ & $(3)$ \\
\cline { 2 - 4 } & & & \\
\hline Standardized set affiliation & $-0.228^{* * *}$ & $-0.332^{* * *}$ & $-0.146^{* *}$ \\
Baseline controls & $(0.071)$ & $(0.076)$ & $(0.070)$ \\
Utilization among & $\mathrm{Y}$ & $\mathrm{Y}$ & $\mathrm{Y}$ \\
proposers & $\mathrm{N}$ & $\mathrm{Y}$ & $\mathrm{Y}$ \\
Proposer count dummies & $\mathrm{N}$ & $\mathrm{N}$ & $\mathrm{Y}$ \\
$N$ & 4,407 & 4,219 & 4,219 \\
Adjusted $R$-squared & 0.329 & 0.332 & 0.348 \\
Sample mean outcome & 4.660 & 4.619 & 4.619 \\
\hline & & & \\
\hline Standardized set affiliation & $-0.219^{* * *}$ & $-0.413^{* * *}$ & -0.082 \\
Baseline controls & $(0.076)$ & $(0.049)$ & $(0.055)$ \\
Utilization among & $\mathrm{Y}$ & $\mathrm{Y}$ & $\mathrm{Y}$ \\
proposers & $\mathrm{N}$ & $\mathrm{Y}$ & $\mathrm{Y}$ \\
Proposer count dummies & $\mathrm{N}$ & $\mathrm{N}$ & $\mathrm{Y}$ \\
$N$ & 4,407 & 4,219 & 4,219 \\
Adjusted $R$-squared & 0.220 & 0.253 & 0.304 \\
Sample mean outcome & 3.067 & 3.071 & 3.071 \\
\hline & & & \\
\hline
\end{tabular}

Note: This table shows results of regressions of survey measures of hard information on standardized set affiliation, based on Equation (7). Survey sample regressions are shown in Panel A, and survey respondent regressions are shown in Panel B. The outcomes are per-specialty measures, constructed by dividing the total survey measures by the number of proposing specialties. Baseline controls are the same as in Column 5 of Table 3. Columns 2 and 3 control for the log annual utilization of the service among all specialties and the log annual utilization of the service among proposing specialties, dropping observations for which these values are missing. Column 3 also includes dummies for the proposing specialty count. Standard errors, clustered by RUC meeting, are in parentheses; ** denotes significance at the $5 \%$ level, and $* * *$ denotes significance at the $1 \%$ level. 


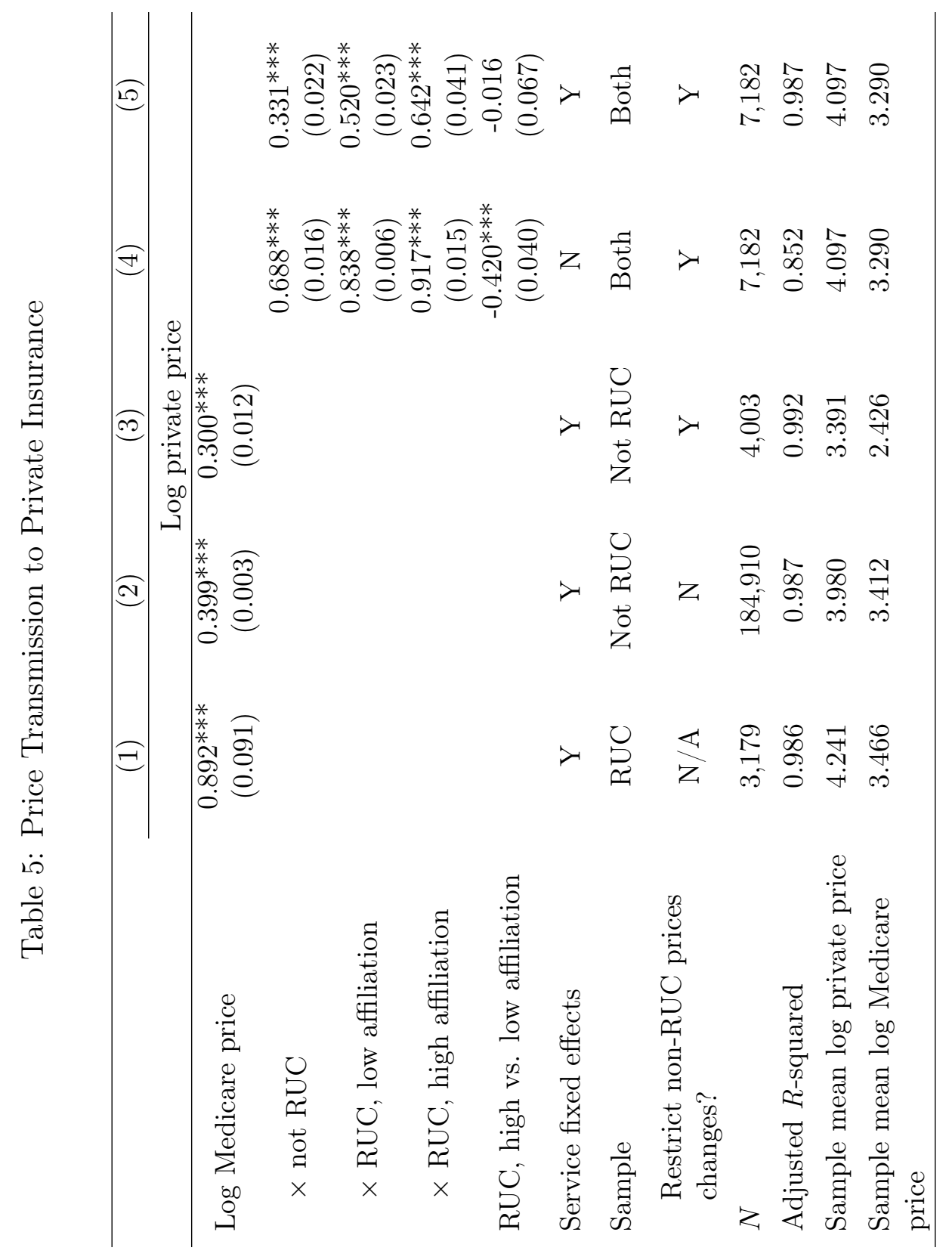


Figure 1: Committee Seats Over Time

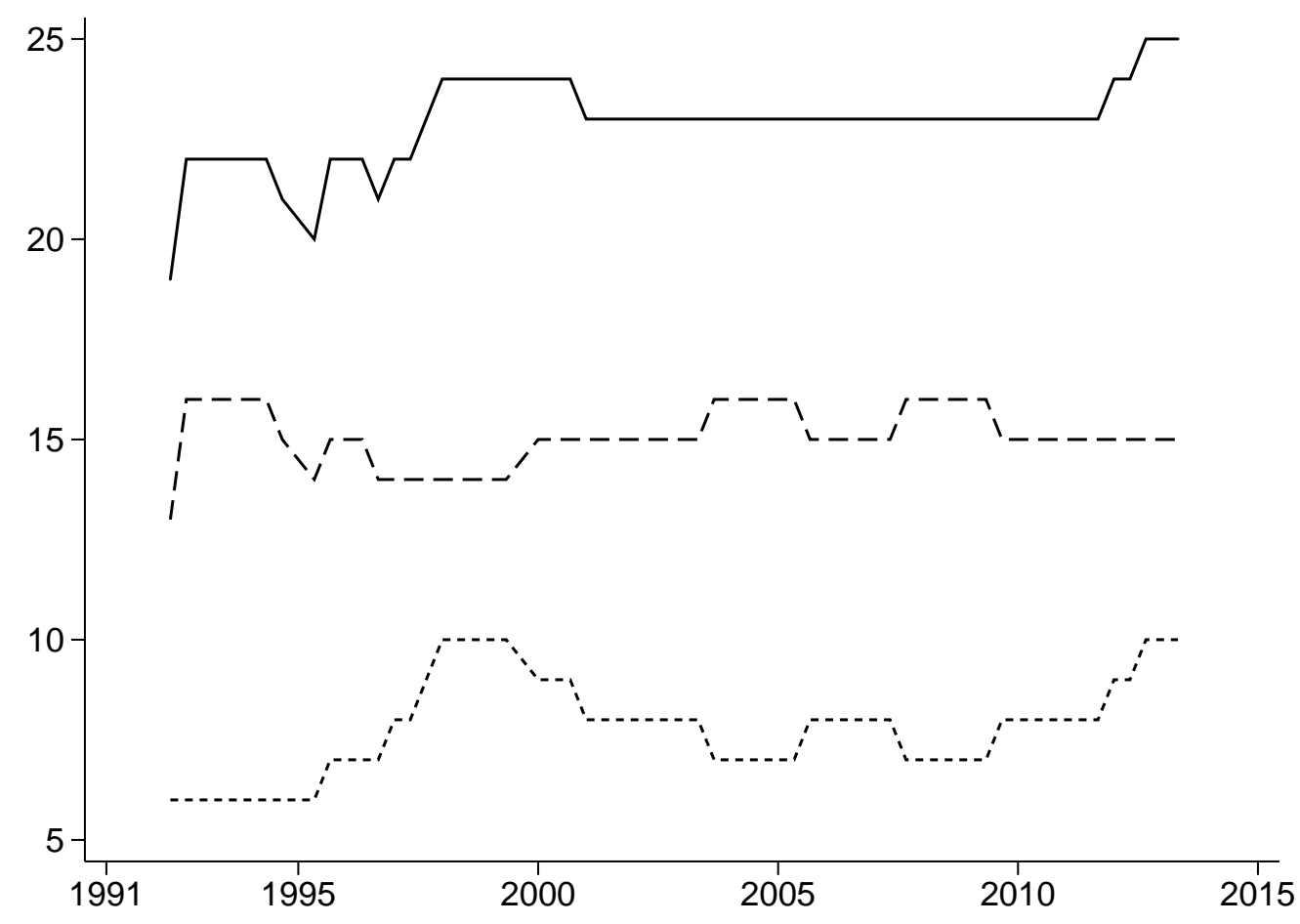

Note: This figures shows the numbers of voting seats on the RUC over time, in total (solid line) and apportioned between "procedural" (dashed line) and "cognitive" (dotted line) specialties. Based on conversations with the RUC, we assign the "procedural" label to general surgery, orthopedic surgery, plastic surgery, ophthalmology, pathology, otolaryngology, dermatology, thoracic surgery, radiology, anesthesiology, gastroenterology, urology, cardiology, obstetrics and gynecology, neurosurgery, pediatric surgery, vascular surgery, radiation oncology, hand surgery, and colorectal surgery. We assign the "cognitive" label to emergency medicine, internal medicine, psychiatry, pediatrics, family medicine, geriatrics, neurology, rheumatology, pulmonary medicine, oncology, infectious disease, and nephrology. 
Figure 2: Affiliation Between Specialties

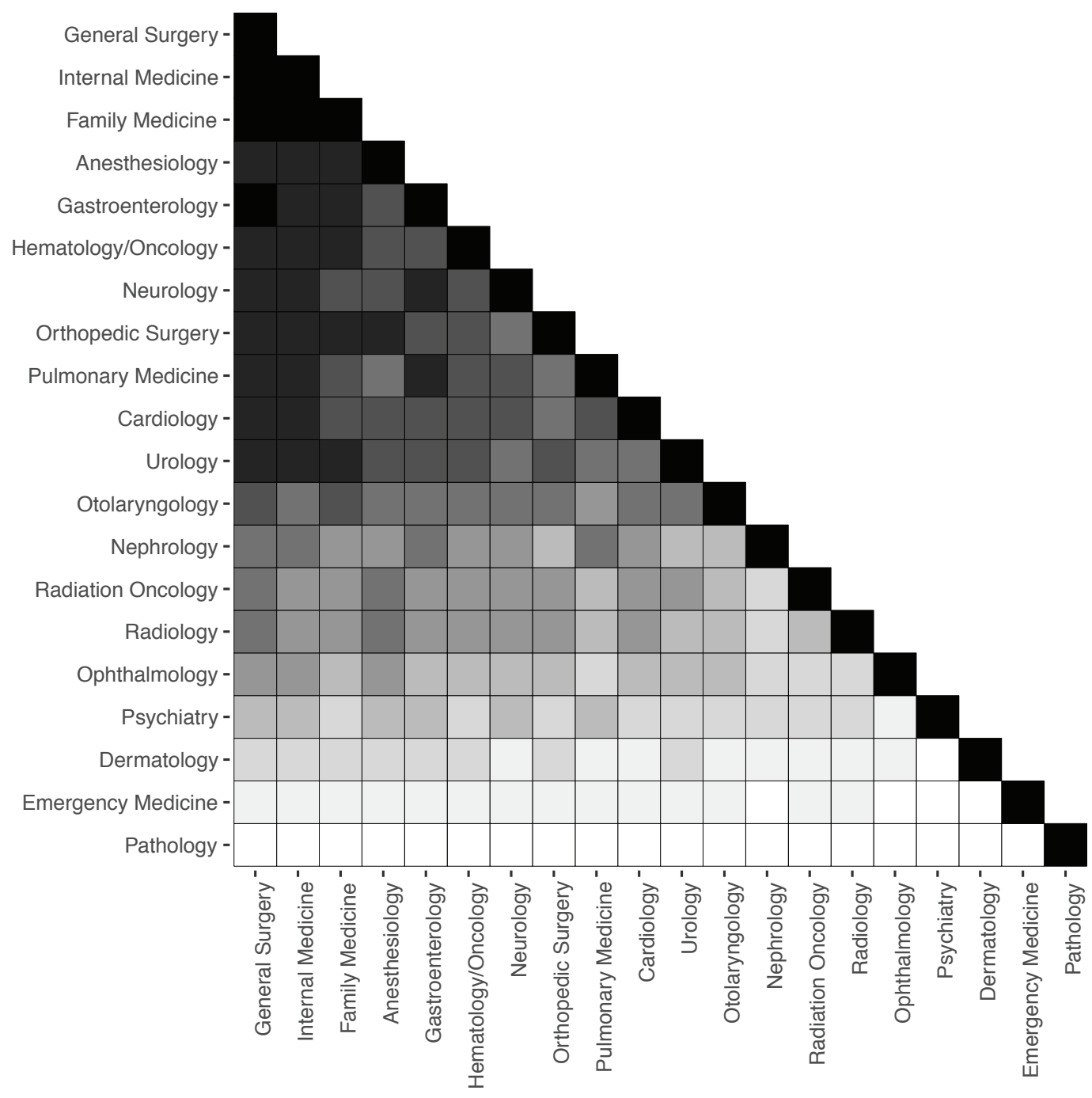

Note: This figure illustrates affiliation between specialties, where the particular formula used is a negative Euclidean distance, described in Equation (3), for the largest 20 specialties. Affiliation values are divided into nine bins with an equal number of specialty pairs. Darker shades signify stronger affiliations. 
Figure 3: Within Specialty Variation in Affiliation
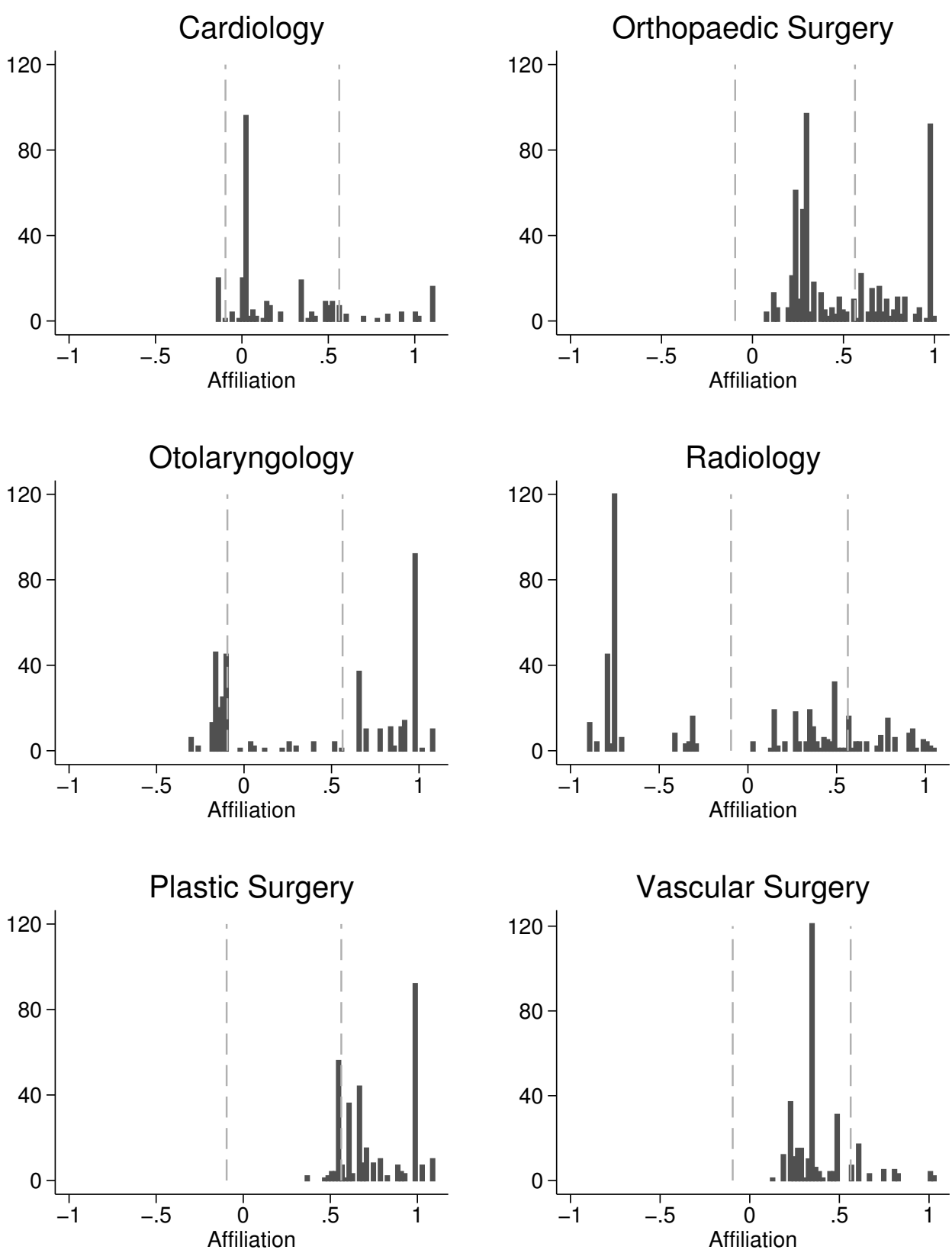

Note: This figure shows examples of within-specialty variation in standardized set affiliation for proposals that are made by one of six specialties. The figure displays in a histogram the distribution of affiliation across proposals within each specialty. Dashed line denote the 25th and 75th percentiles of affiliation overall. 
Figure 4: Balance of Medicare Beneficiary Characteristics across Affiliation

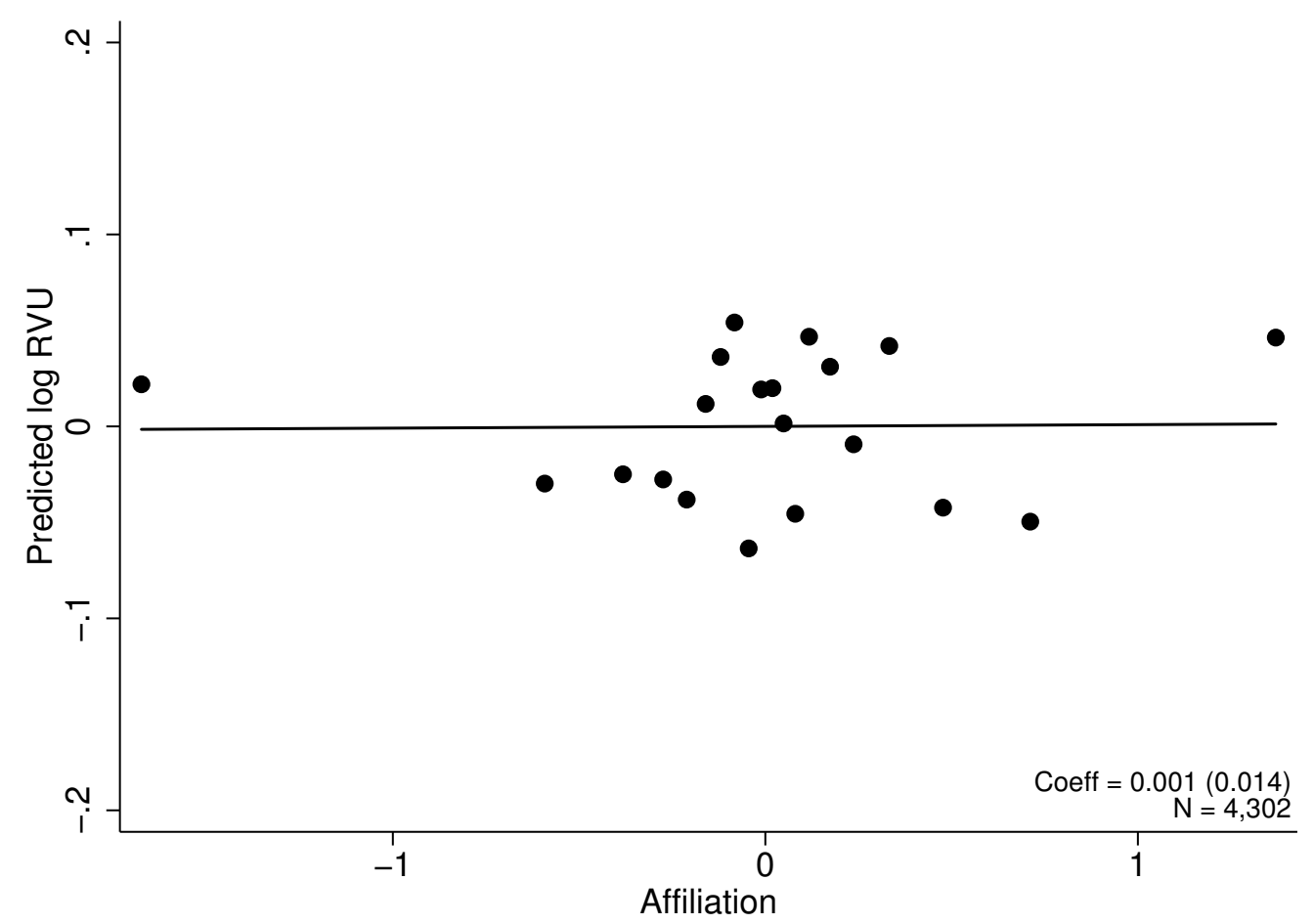

Note: This figure is a binned scatterplot of residual predicted log RVU, based on Medicare beneficiary characteristics, on residual affiliation, where each dot represents $5 \%$ of the data, ordered by residual affiliations. Log RVU is first predicted by Medicare beneficiary characteristics, which are listed in Table 2 . The $R$-squared of this prediction equation is 0.249 . Residuals are formed by regressing predicted log RVU and affiliation, respectively, on meeting dummies and specialty shares $\mathbf{w}_{i}$. The line shows the best fit through the residualized data, with corresponding coefficient and standard error clustered by meeting. 
Figure 5: Random Timing of Proposals

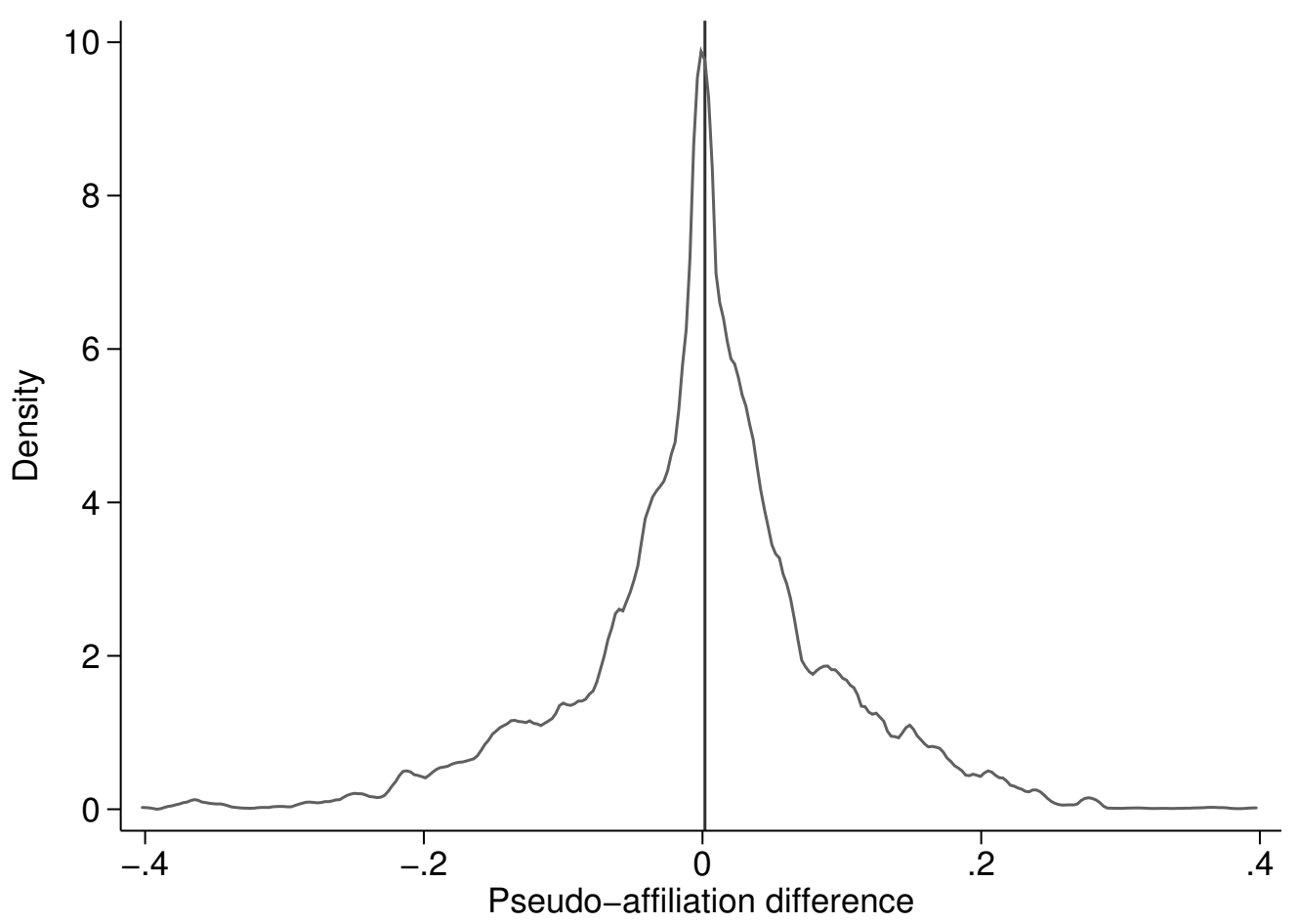

Note: This figure show the distribution of the difference between set affiliation in pseudo-meetings and the actual set affiliation of each proposal. The distribution therefore includes 59 pseudo-affiliation measures corresponding to pseudo-meetings for each of 4,432 proposals. The mean of these observations, 0.0017 , is shown with the vertical line. The standard deviation of the distribution is 0.0898 , which implies that the standard error of the mean at the proposal level is 0.0013 . 
Figure 6: Balance of Proposal Probability on Predicted Price

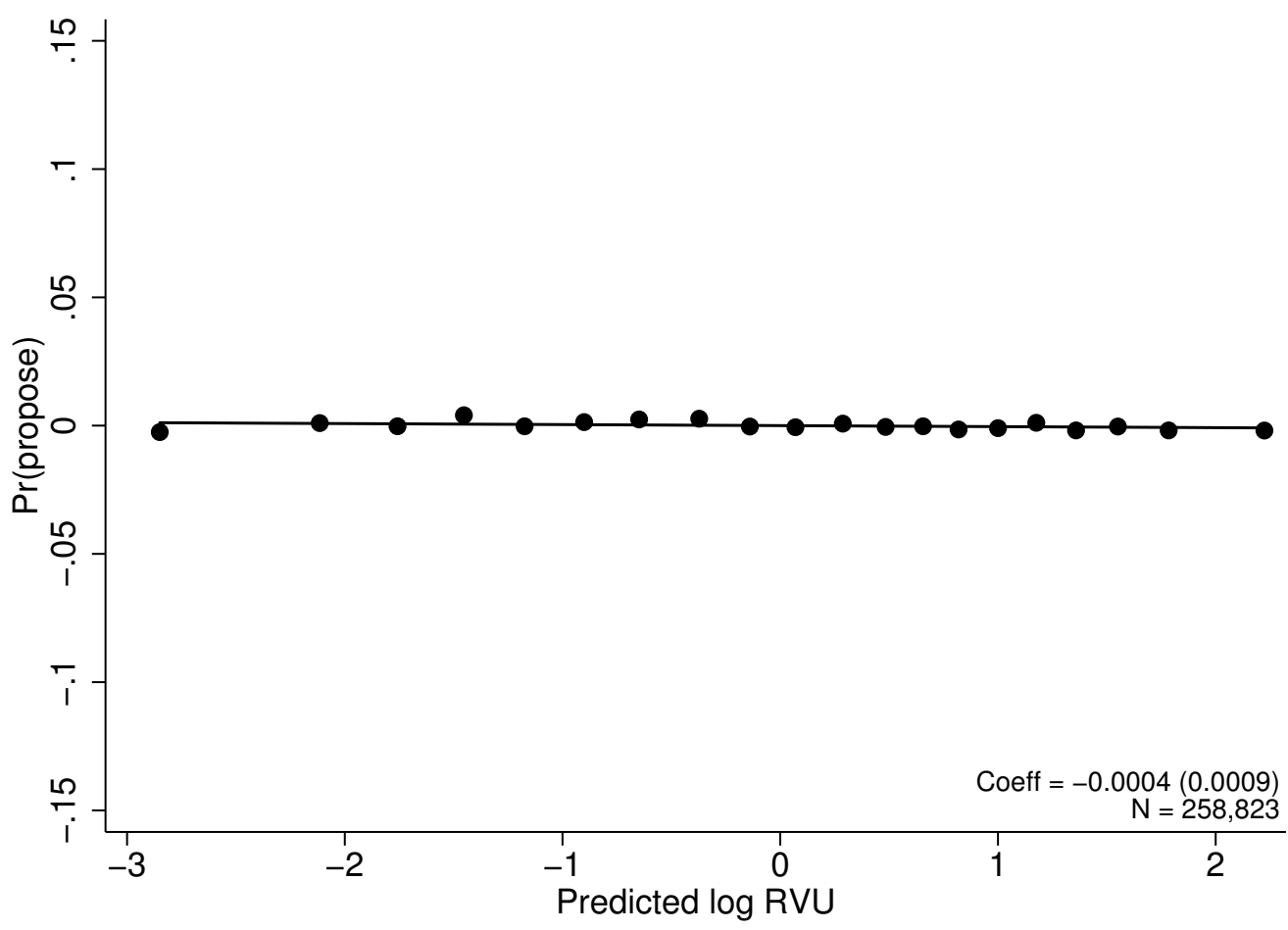

Note: This figure is a binned scatterplot of residual proposal probability on residual predicted log RVU, where each dot represents $5 \%$ of the data, ordered by residual predicted log RVU. Each observation is a proposal-specialty pair, and the outcome variable of interest is an indicator for whether the specialty was part of that proposal. Log RVU is predicted from service (CPT code) characteristics, word descriptions, and prior RVU, which are described in Table 3 ; the prediction equation has an adjusted $R$-squared of 0.88 . The specialty proposal indicator and predicted log RVU are both residualized by the proposal propensity, which is calculated by a logit model of flexible functions of $\mathbf{w}_{i}$ and specialty dummies alone. This propensity model has a pseudo- $R$-squared of 0.67 . The standard deviation of the proposal propensities across proposal-specialty pairs is 0.13 , so that the span of the $y$-axis is approximately 1 standard deviation above and below. The line shows the best fit through the residualized data, with corresponding coefficient and standard error clustered by meeting. 
Figure 7: Affiliation Effect on Relative Price

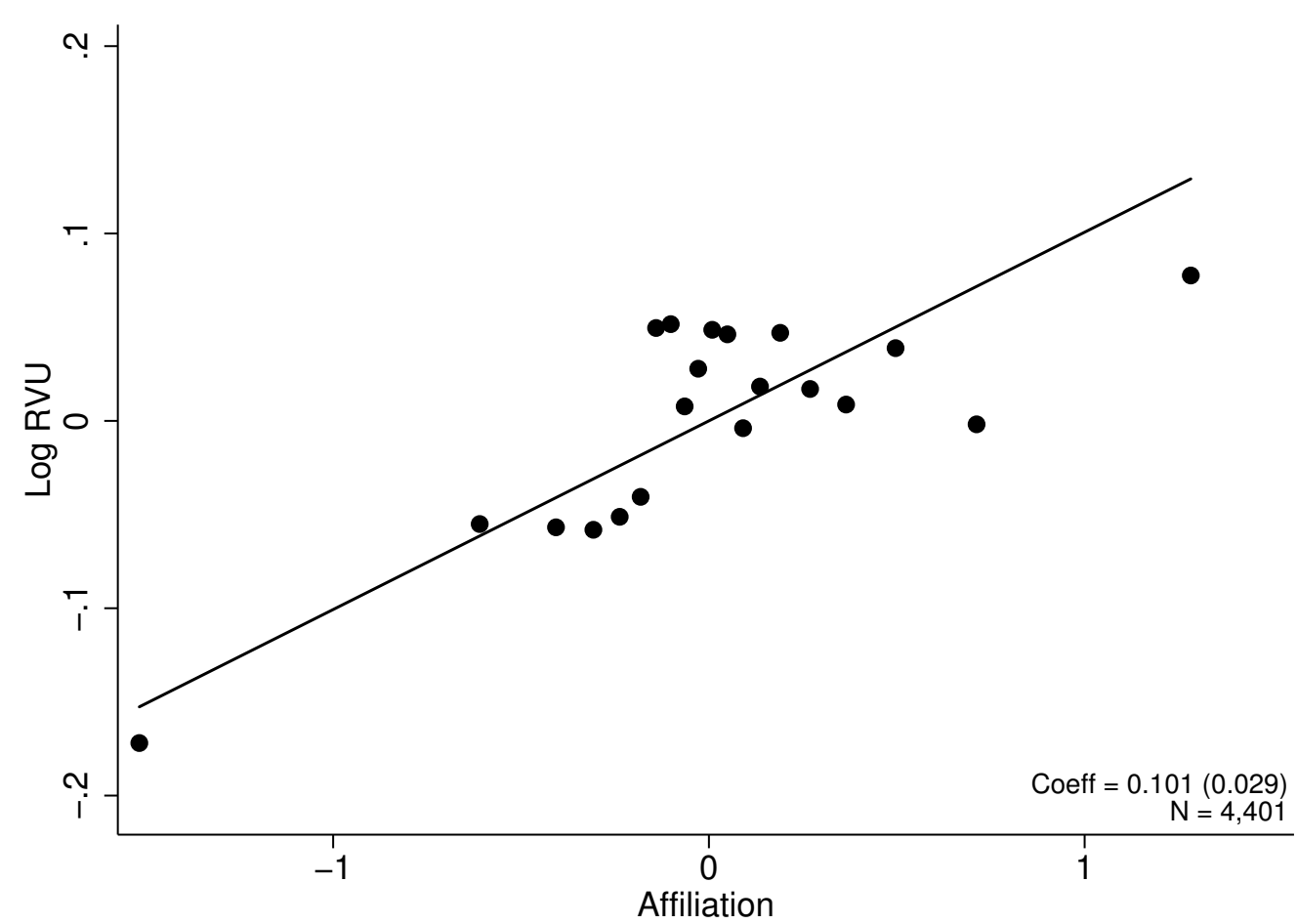

Note: This figure is a binned scatterplot of residual log RVU on residual affiliation, where each dot represents $5 \%$ of the data, ordered by residual affiliations. Residuals are formed by regressing log RVU and affiliation, respectively, on controls specified in Column 4 of Table 3. The line shows the best fit through the residualized data, and the slope corresponds to the estimated coefficient of interest $\alpha$ in Equation (6), with standard errors clustered by RUC meeting. 
Figure 8: Revenue Reallocation with Equal Affiliation
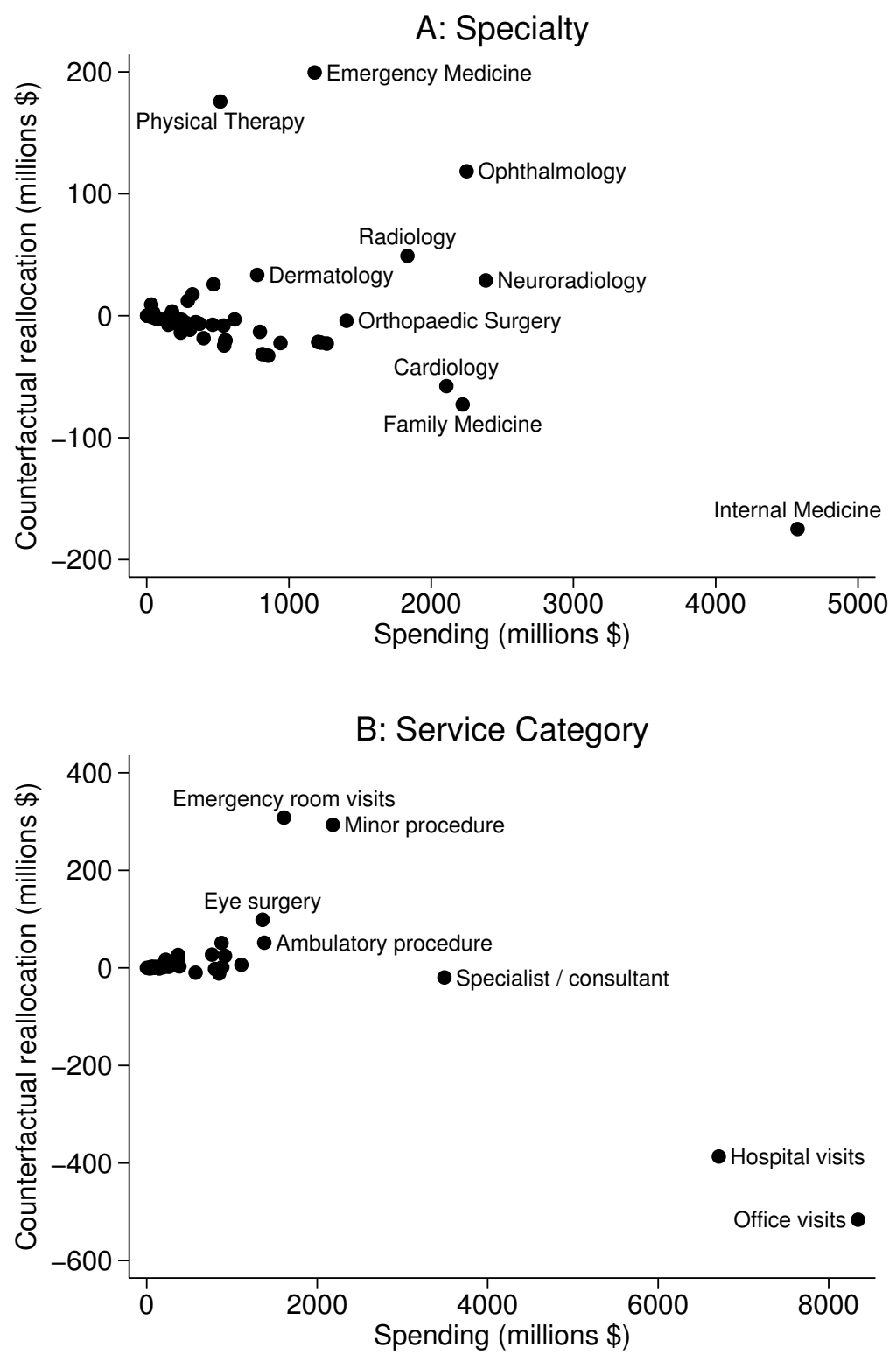

Note: This figure shows counterfactual yearly revenue reallocation by equalizing the affiliation of all proposals in each year. Panel A shows reallocation across specialties; Panel B shows reallocation aggregated across Berenson-Eggers Type of Service (BETOS) categories. Average annual spending for each specialty is on the $x$-axis, while the counterfactual reallocation setting affiliation to the mean for all proposals is on the $y$-axis. Utilization quantities for each service (CPT code) is held fixed, and the annual Medicare budget for physician work is set at $\$ 70$ billion $\times 51 \%=\$ 35.7$ billion. Details are given in Section 4.2 . 
Figure 9: Revenue Reallocation with Proportional RUC Representation

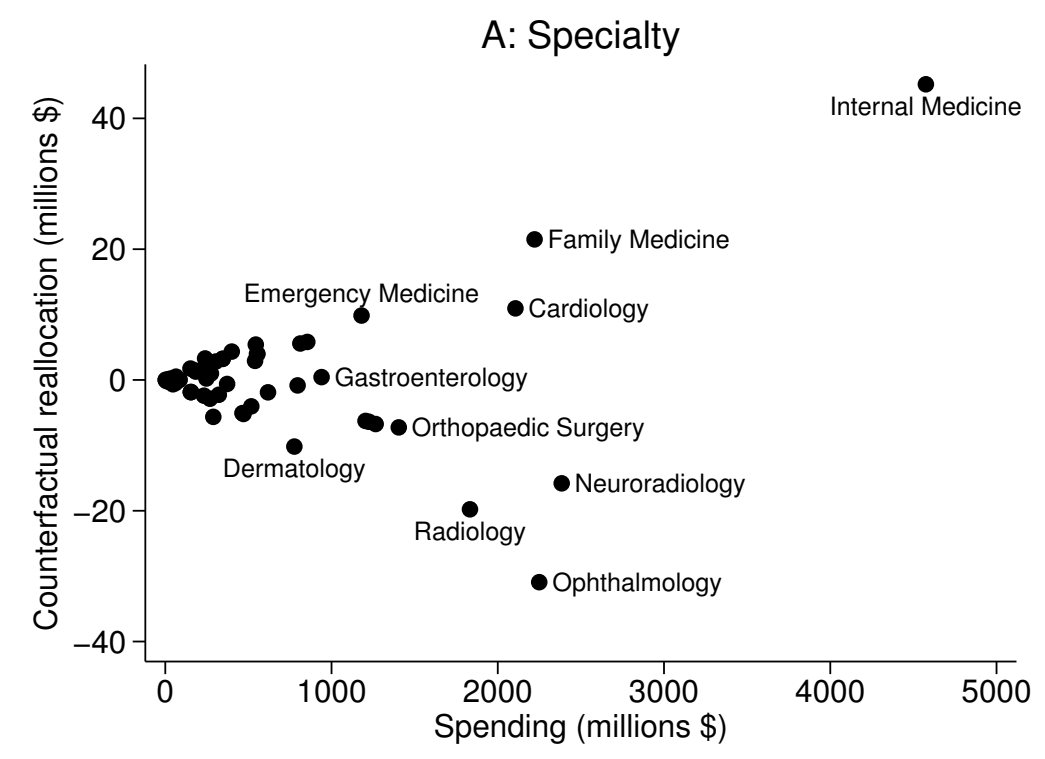

B: Service Category

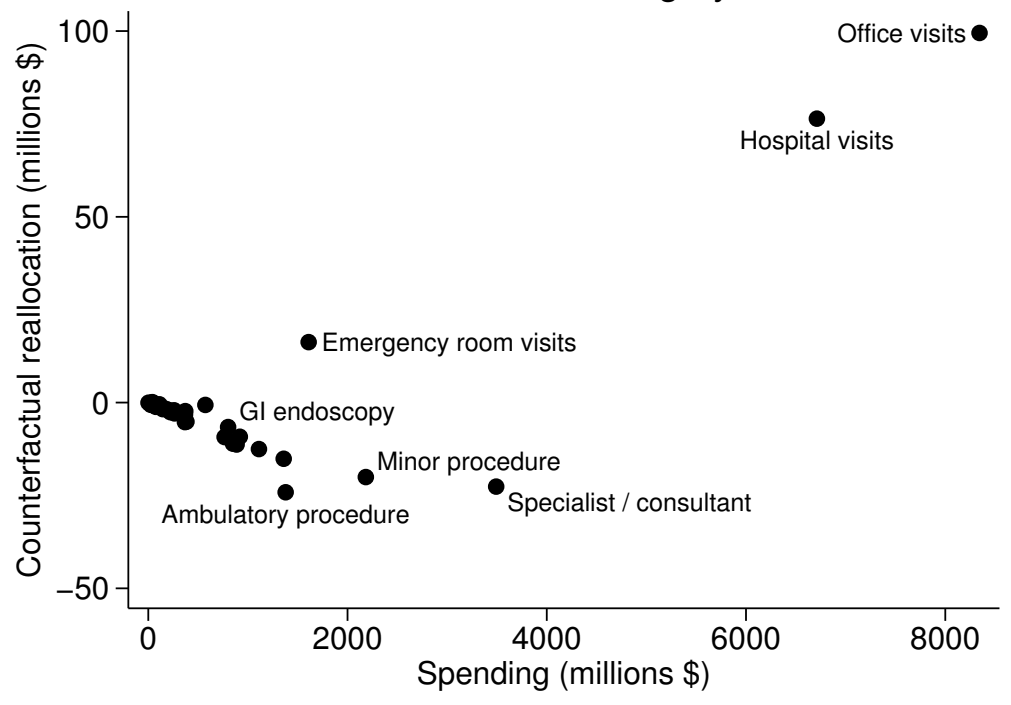

Note: This figure shows counterfactual yearly revenue reallocation by changing the RUC membership to be constant and proportional to the population of physician specialties in the US, as given in Table A-5. Panel A shows reallocation across specialties; Panel B shows reallocation aggregated across BerensonEggers Type of Service (BETOS) categories. Average annual spending for each specialty is on the $x$-axis, while the counterfactual reallocation setting affiliation to the mean for all proposals is on the $y$-axis. Utilization quantities for each service (CPT code) is held fixed, and the annual Medicare budget for physician work is set at $\$ 70$ billion $\times 51 \%=\$ 35.7$ billion. Details are given in Section 4.2 . 
Figure 10: Affiliation Effect on Hard Information

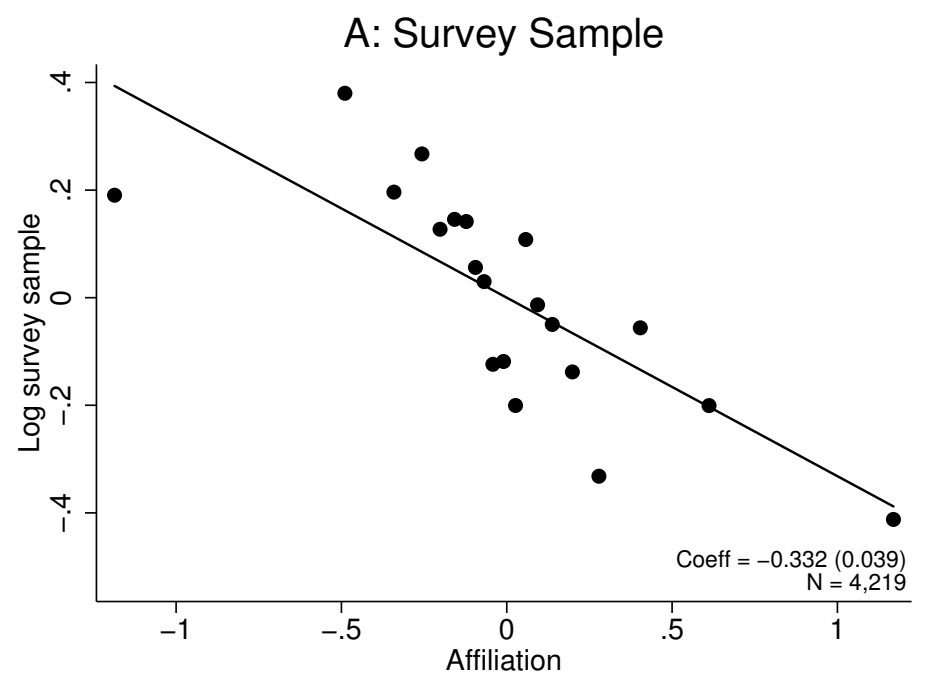

B: Respondents

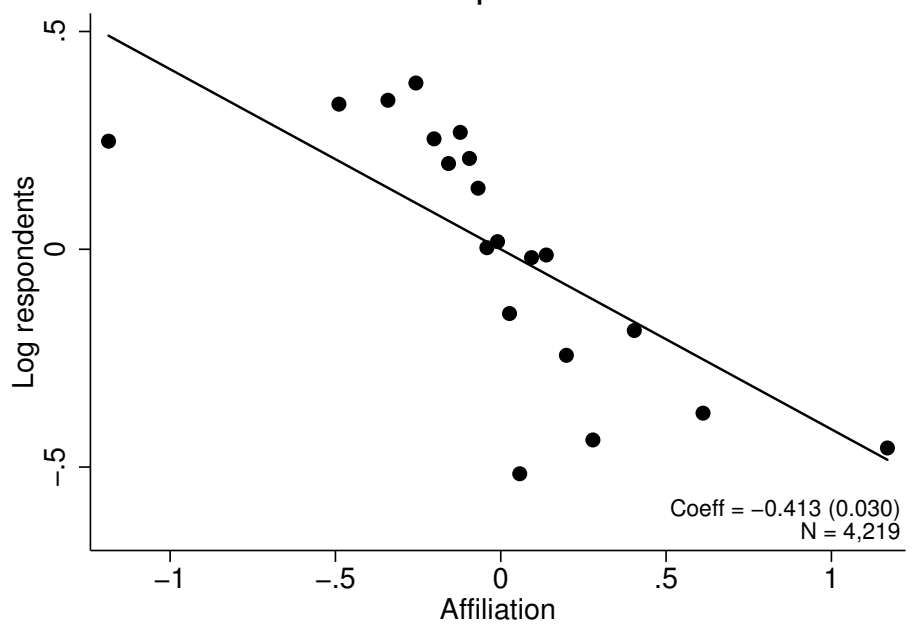

Note: This figure is a binned scatterplot of the residual log per-specialty survey sample (Panel A) and log per-specialty survey respondents (Panel B) on residual affiliation, where each dot represents $5 \%$ of the data, ordered by residual affiliations. We form residuals by regressing the survey variables of interest and affiliation on the controls specified in Column 3 of Table 4. Lines show the best fit through the residualized data, and the line slopes correspond to the estimated coefficient of interest $\alpha$ in Equation (7), with standard errors clustered by RUC meeting. 
Figure 11: Price Transmission to Private Insurance

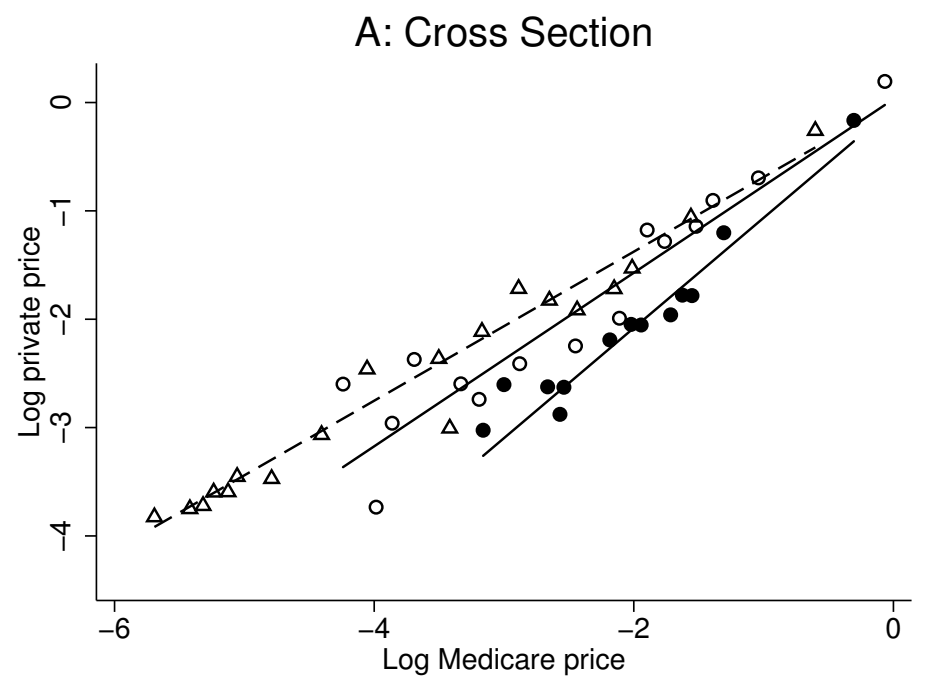

B: Within Service

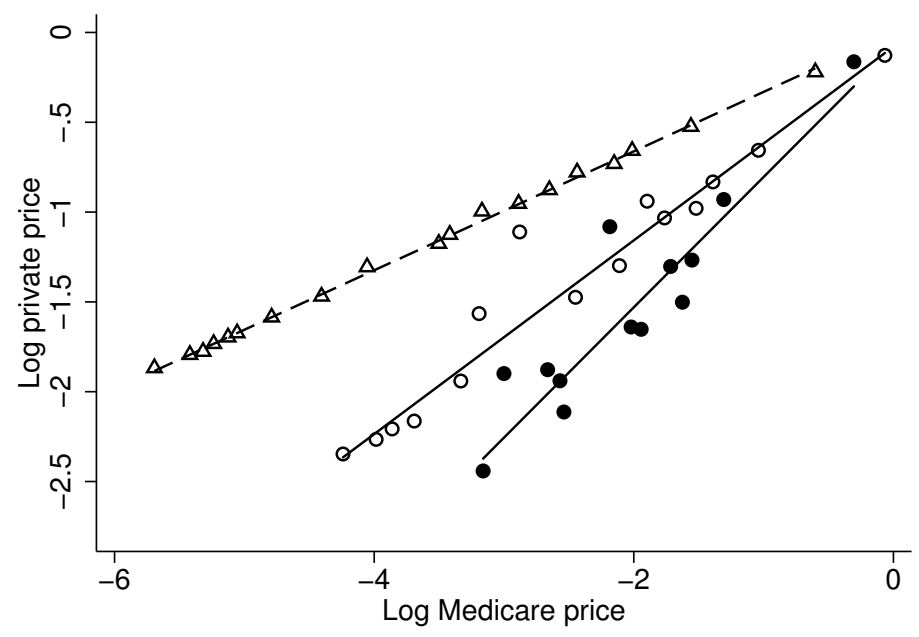

Note: This figure is a binned scatterplot of the relationship between normalized log Medicare price and normalized log private price, as described in the note for Table 5. Panel A shows the relationship without controlling for service (CPT code) and corresponds to Column 4 of Table 5, while Panel B shows this relationship controlling for CPT code and corresponds to Column 5 of Table 5. In each panel, residuals of the relevant regression are added to predictions of normalized log private price based on normalized log Medicare price and the following Medicare price categories: not associated with RUC proposal (triangles), associated with RUC proposal with lower affiliation (hollow circles), and associated with RUC proposal with higher affiliation (solid circles). Each marker represents $5 \%$ of the data conditional on the relevant Medicare price category. Lines show the best fit through the markers and by construction have slopes equivalent to the relevant interaction terms in Table 5. 


\section{Appendix}

\section{A-1 Setting the Medicare Budget}

This appendix summarizes the process that sets the overall Medicare budget for physician services, which equivalently determines the conversion factor, or $\mathrm{CF}_{t}$ in Equation (1). We focus on the period between the Balanced Budget Act of 1997 and the Medicare Access and CHIP Reauthorization Act of 2015. A more extensive discussion of this process can be found elsewhere (e.g., American Medical Association, 2015; Centers for Medicare and Medicaid Services, 2014). During this period, CMS set $\mathrm{CF}_{t}$ according to this formula:

$$
\mathrm{CF}_{t}=\mathrm{CF}_{t-1} \times\left(1+\mathrm{MEI}_{t}\right) \times\left(1+\mathrm{UAF}_{t}\right) \times \mathrm{BN}_{t}
$$

where $\mathrm{MEI}_{t}$ is the Medicare Economic Index, $\mathrm{UAF}_{t}$ is the Update Adjustment Factor, and $\mathrm{BN}_{t}$ is the Budget Neutrality adjustment.

$\mathrm{MEI}_{t}$ is the weighted-average price change for inputs required to operate a self-employed physician practice in the United States. The measure indexes inflation for medical services. There are two broad categories of inputs: the physician's own time and his or her practice expense. The MEI Technical Advisory Panel continually reviews and updates the index, recommending changes to ensure that $\mathrm{MEI}_{t}$ appropriately meets its statutory purpose.

$\mathrm{UAF}_{t}$ is a mechanism that keeps Medicare spending at an acceptable level given real gross domestic product per capita and year-to-year changes in fees and beneficiaries. The current year's target expenditures are equal to target expenditures in the previous year adjusted by the Sustainable Growth Rate $\left(\mathrm{SGR}_{t}\right)$. The update also compares actual expenditures with target expenditures from April 1, 1996 through the preceding year. By federal statute, $\mathrm{UAF}_{t} \in$ $[-7 \%, 3 \%]$, and the formula for the $\mathrm{UAF}_{t}$ is based on the following identities, relating target and actual spending:

$$
\begin{aligned}
\sum_{t^{\prime}=1}^{t} \text { Target }_{t^{\prime}} & =\sum_{t^{\prime}=1}^{t} \text { Actual }_{t^{\prime}} \\
\text { Actual }_{t} & =\text { Actual }_{t-1} \times\left(1+\mathrm{SGR}_{t}\right) \times\left(1+\mathrm{UAF}_{t}\right) \\
\text { Target }_{t} & =\text { Target }_{t-1} \times\left(1+\mathrm{SGR}_{t}\right)
\end{aligned}
$$

These identities yield

$$
\mathrm{UAF}_{t}=\frac{\text { Target }_{t-1}-\text { Actual }_{t-1}}{\text { Actual }_{t-1}} \times 0.75+\frac{\sum_{t^{\prime}=1}^{t-2}\left(\text { Target }_{t^{\prime}}-\text { Actual }_{t^{\prime}}\right)}{\text { Actual }_{t-1} \times\left(1+\mathrm{SGR}_{t}\right)} \times 0.33,
$$

after being modified by "dampening" weights of 0.75 and 0.33 , between components from the previous year and all other years before that, respectively. 
The Sustainable Growth Rate $\left(\mathrm{SGR}_{t}\right)$ used above is calculated according to four factors: (i) the estimated percentage change in fees for physicians' services, (ii) the estimated percentage change in the average number of Medicare fee-for-service beneficiaries, (iii) the estimated 10year average annual percentage change in real gross domestic product per capita, and (iv) the estimated percentage change in expenditures due to changes in law or regulations.

The Budget Neutrality adjustment offsets expenditure changes that result from updates to the relative value units of medical services and ensures that RVU inflation does not change the Medicare budget:

$$
\mathrm{BN}_{t}=\frac{\sum_{i} R V U_{i, t-1} \times q_{i, t-1}}{\sum_{i} R V U_{i, t} \times q_{i, t-1}},
$$

which is closely related to the condition in Equation (A-5.4) we use in simulating counterfactual revenue in Section 4.2. Historically, $\mathrm{BN}_{t}$ adjustments have been relatively minor considerations in setting $\mathrm{CF}_{t}$, compared to $\mathrm{MEI}_{t}$ and $\mathrm{UAF}_{t}$. Changes to the relative value of medical services via $\mathrm{BN}_{t}$ are also limited by statute to $\$ 20$ million annually.

Despite scheduled reductions in the CF according to the SGR formula, the most recent year with a CF reduction was 2002. Since then, Congress has annually overridden scheduled reductions (colloquially known as the "doc fix"). Most recently, the Medicare Access and CHIP Reauthorization Act of 2015 removed the SGR formula used to determine the CF. In its place, the act provided a half-percent increase in the physician fee schedule rate until 2020 (Clough and McClellan, 2016).

\section{A-2 Measuring Affiliation}

In this appendix, we provide an economic foundation for measuring affiliation as a negative distance in the space of quantity shares or revenue shares. We then discuss our choice of distance metric, providing context with other efforts in the economics literature to quantify relationships between groups.

\section{A-2.1 Specialty Objective Rationale for Vector Space}

Our goal for measuring affiliation is to quantify the degree to which specialties with many interests may share preferences (Caillaud and Tirole, 2007). A natural benchmark for a specialty's objective is its revenue. Consider the revenue of specialty $s$ as

$$
R_{s}=\sum_{i} p_{i} q_{i s}
$$

or the sum of revenues from each service $i$, which in turn is the price of $i, p_{i}$, multiplied by the quantities of $i$ provided by $s, q_{i s}$.

The interests of two specialties then may be linked via prices changes in several ways. A 
change in price for any service may lead to changes in the price of many other services, because by nature, prices are relative and can be used to justify prices of comparable services. Further, the fixed budget implies that any given price change mechanically affects all prices. Finally, if physicians (or patients) are responsive to price in their decisions, a price change or changes can lead to changes in quantities provided (and demanded) of the service undergoing the price change. The quantities of substitute or complementary services may adjust as well.

To capture this combination of mechanisms, we can write the first-order effect of a vector $\mathbf{p}$ of price changes on $R_{s}$ as

$$
\frac{d R_{s}}{d \mathbf{p}}=\sum_{i}\left(q_{i s} \frac{d p_{i}}{d \mathbf{p}}+p_{i} \frac{d q_{i s}}{d \mathbf{p}}\right)
$$

While $q_{i A}$ and $p_{i}$ are observed, $d p_{i} / d \mathbf{p}$ and $d q_{i s} / d \mathbf{p}$ are generally unknown. In order to capture similarity in the effects of $\mathbf{p}$ on the revenue of two different specialties, as in Equation (A-2.1), we need to make simplifying assumptions. We discuss two such assumptions below that allow us to link the vector space of a distance metric to alignment between two specialties' objectives.

\section{A-2.1.1 Quantity Shares}

Under the assumption of fixed quantities (i.e., quantities are completely inelastic to price), the derivative in Equation (A-2.1) reduces to

$$
\frac{d R_{s}}{d \mathbf{p}}=\sum_{i} q_{i s} \frac{d p_{i}}{d \mathbf{p}}
$$

Further, fixed quantities allow us to scale revenue to be per-service; we can then compare specialties of different overall volume:

$$
\frac{d r_{s}}{d \mathbf{p}}=\sum_{i} \sigma_{i s}^{q} \frac{d p_{i}}{d \mathbf{p}}
$$

where $r_{s} \equiv R_{s} / \sum_{i} q_{i s}$ is the per-service revenue, and $\sigma_{i s}^{q} \equiv q_{i s} / \sum_{i} q_{i s}$ is the quantity share of $i$ relative to other procedures that $s$ performs.

The difference in the effect on per-service revenue between specialties $A$ and $B$ is

$$
\frac{d r_{A}}{d \mathbf{p}}-\frac{d r_{B}}{d \mathbf{p}}=\sum_{i}\left(\sigma_{i A}^{q}-\sigma_{i B}^{q}\right) \frac{d p_{i}}{d \mathbf{p}} .
$$

Distances in the vector space of quantity shares, i.e., $\left(\sigma_{A}^{q}, \sigma_{B}^{q}\right)$, thus capture this difference for any arbitrary set of price changes (i.e., any arbitrary $\mathbf{p}$ and the corresponding $d p_{i} / d \mathbf{p}$ for all $i$ ).

In addition, this difference in derivatives equivalently represents differences in the response of per-service profit, where "profit" is price minus a concept of service-specific cost. ${ }^{46}$ For any arbitrary set of procedure costs that vary by $i$ and specialty, the derivative of profit with respect

\footnotetext{
${ }^{46}$ This cost can be a cost of effort, a financial cost, or an opportunity cost, such as when time used to perform service $i$ detracts from time performing other procedures.
} 
to price changes remains the same.

\section{A-2.1.2 Revenue Shares}

If we instead assume that quantities are allocated across specialties in fixed proportion, then we may use the vector space of revenue shares to measure similarity in percentage change in revenue. To see this, first assume that $q_{i s}=w_{i s} q_{i}$, where $w_{i s}$ is fixed regardless of $q_{i} \equiv \sum_{s} q_{i s}$. Then note that percentage revenue change is

$$
\begin{aligned}
\frac{d R_{s} / d \mathbf{p}}{R_{s}} & =\frac{1}{R_{s}} \sum_{i}\left(q_{i s} \frac{d p_{i}}{d \mathbf{p}}+p_{i} \frac{d q_{i s}}{d \mathbf{p}}\right) \\
& =\sum_{i} \frac{q_{i s} p_{i}}{R_{s}} \cdot \frac{q_{i s} \cdot d p_{i} / d \mathbf{p}+p_{i} \cdot d q_{i s} / d \mathbf{p}}{p_{i} q_{i s}} \\
& =\sum_{i} \sigma_{i s}^{R} \cdot \frac{q_{i} \cdot d p_{i} / d \mathbf{p}+p_{i} \cdot d q_{i} / d \mathbf{p}}{p_{i} q_{i}}, \\
& =\sum_{i} \sigma_{i s}^{R} \cdot \frac{d\left(p_{i} q_{i}\right) / d \mathbf{p}}{p_{i} q_{i}},
\end{aligned}
$$

where $\sigma_{i s}^{R} \equiv\left(p_{i} q_{i s}\right) / R_{s}$ is the quantity share of $i$ relative to other procedures that $s$ performs, and the third line derives from dividing $w_{i s}$ from the numerator and the denominator. The term multiplying $\sigma_{i s}^{R}$ is a constant for each service $i$; it does not depend on the identity of $s$.

The difference in the percentage revenue change between specialties $A$ and $B$ is then

$$
\frac{d R_{A} / d \mathbf{p}}{d R_{A}}-\frac{d R_{B} / d \mathbf{p}}{d R_{B}}=\sum_{i}\left(\sigma_{i A}^{R}-\sigma_{i B}^{R}\right) \frac{d\left(p_{i} q_{i}\right) / d \mathbf{p}}{p_{i} q_{i}}
$$

Distances in the vector space of revenue shares, i.e., $\left(\sigma_{i A}^{R}, \sigma_{i B}^{R}\right)$, correspondingly capture this difference in percentage revenue changes.

Whether this is a meaningful vector space for distance depends on the plausibility of the assumption that quantities for a given service $i$ are allocated across specialties in fixed proportions, regardless of $q_{i}$. In addition, this vector space does not allow us to measure similarity in specialty interests other than revenue. In particular, measures in this vector space would not capture any concept of specialty profit.

\section{A-2.2 Distance Metrics}

In economics, several threads of literature have developed quantitative measures of relationships between groups. The literature on segregation has developed measures of isolation and dissimilarity to reflect the interaction between groups (White, 1986; Cutler et al., 1999; Gentzkow and Shapiro, 2011; Esteban et al., 2012). Another literature on technological spillovers has sought to measure the likelihood that productive entities in multiple fields may affect each other (Jaffe, 
1986; Bloom et al., 2013). In our application, we seek to measure closeness in preferences between specialties with many interests.

This goal motivates our choice of using distance metrics in vector spaces (quantity and revenue shares) that reflect specialties' interests. ${ }^{47}$ As our baseline distance metric, we use the standard Euclidean distance:

$$
a\left(s_{A}, s_{B}\right)=-\sqrt{\left(\sigma_{A}-\sigma_{B}\right)\left(\sigma_{A}-\sigma_{B}\right)^{\prime}}
$$

where $\sigma_{s}$ is the vector of quantity or revenue shares for specialty $s$ across services or BETOS categories of services. The Euclidean distance between the two vectors $\sigma_{A}$ and $\sigma_{B}$ corresponds to the difference in total effects on the revenue of the two specialties, particularly if the component effect from each service- $d p_{i} / d \mathbf{p}$ or $\frac{d\left(p_{i} q_{i}\right) / d \mathbf{p}}{p_{i} q_{i}}$ on quantity or revenue shares, respectively-is orthogonal (independent) and equal (identical).

We also consider a Euclidean distance metric weighted by the Gini coefficient of each service:

$$
a\left(s_{A}, s_{B}\right)=-\sqrt{\left(\sigma_{A}-\sigma_{B}\right) \mathbf{G}\left(\sigma_{A}-\sigma_{B}\right)^{\prime}},
$$

where $\mathbf{G}$ is a diagonal weighting matrix, such that $G(i, i)=G_{i}$ is the Gini coefficient across $\sigma_{i s}$ for services $i$, which places more weight on services that differ in shares across specialties. This Gini-weighted metric will naturally result in greater variation in distances.

Finally, we consider Manhattan distance, in $L_{1}$ space:

$$
a\left(s_{A}, s_{B}\right)=-\sum_{i}\left|\sigma_{i A}-\sigma_{i B}\right|
$$

In addition to these distance metrics, we also consider two measures similar to correlation notions, akin to those in the literature on technology spillovers (Jaffe, 1986; Bloom et al., 2013): angular distance and correlation. Angular distance is in arc space, with affiliation

$$
a\left(s_{A}, s_{B}\right)=-\frac{2}{\pi} \cos ^{-1}\left(\frac{\sigma_{A} \cdot \sigma_{B}}{\sqrt{\sigma_{A} \cdot \sigma_{A}} \sqrt{\sigma_{B} \cdot \sigma_{B}}}\right) .
$$

Our correlation measure is

$$
a\left(s_{A}, s_{B}\right)=-\frac{\sum_{i}\left(\sigma_{i A}-\bar{\sigma}_{A}\right)\left(\sigma_{i B}-\bar{\sigma}_{B}\right)}{\sqrt{\sum_{i}\left(\sigma_{i A}-\bar{\sigma}_{A}\right)^{2} \sum_{i}\left(\sigma_{i B}-\bar{\sigma}_{B}\right)^{2}}},
$$

which is not a distance metric, as it does not satisfy the triangle inequality property. These

\footnotetext{
${ }^{47}$ In addition to quantity shares and revenue shares based on individual services defined by CPT codes, we also consider quantity shares and revenue shares in 107 Berenson-Eggers Type of Service (BETOS) categories. This formulation is more restrictive but uses prior knowledge to group services into categories that likely covary. In this sense, this vector space may improve the characterization of affiliation if BETOS categories capture a sufficiently large amount of information about CPT codes in terms of the price or quantity effects of p. On the other hand, if there remains substantial heterogeneity in effects within BETOS categories, then affiliation measures based on this vector space will perform less well.
} 
metrics, along with Manhattan distance, are also often invoked in the statistical and computer science literatures in settings in which sampling noise over many features can be magnified by Euclidean distances (Aggarwal et al., 2001). In our setting, however, we perfectly observe Medicare quantity shares (from the universe of data) and prices and therefore do not share this concern. Furthermore, and more importantly, because both angular distance and correlation normalize vectors to have a length of 1 , they may distort the meaning of elements of $\sigma_{s}$ away from shares: ${ }^{48}$ A large difference in the quantity or revenue share of a particular service is a meaningful economic concept, while a re-normed element of $\sigma_{s}$ has no meaningful interpretation.

\section{A-3 Mixed Strategies in Endogenous Proposals}

In this appendix, we sketch a simple signaling model of proposals to provide intuition for the random variation we observe in the endogenous decisions of specialties to propose. As in our main conceptual framework, in Section 5.1, we assume a specialty society may be biased, but for tractability, we rule out any downstream communication or any potential bias of the RUC. ${ }^{49}$ The first important feature of the model is that proposals to the RUC are costly. Second, if there is more than one proposing specialty that would have proposed alone, then there cannot be a unique (or symmetric) pure strategy equilibrium that determines specialty proposals. In other words, if specialty societies cannot fully coordinate, then we will have random variation in the identities of proposing specialties. In this sketch, we ignore the possibility that costs may vary, often quasi-randomly, in order to clarify the latter source of random variation.

Specifically, consider specialty society utility

$$
u_{S}=-\left(\theta+b_{S}-p\right)^{2}-c D_{S}
$$

where $\theta \in\{0,1\}$ is the true price, $b_{S}>0$ is the specialty's bias, $p$ is the price recommended by the RUC (and set by the government), $c$ is the cost of proposing, and $D_{S} \in\{0,1\}$ is an indicator for the specialty proposing. The RUC's (and the government's) utility is $u_{R}=-(\theta-p)^{2}$. We assume that $\operatorname{Pr}(\theta=1)=\frac{1}{2}$.

In a separating pure strategy equilibrium with a single specialty, the specialty will propose if and only if $\theta=1$, and the government will set $p=D_{S}$. The specialty must then have bias $b_{S} \in\left[\frac{c-1}{2}, \frac{c+1}{2}\right]$. If bias is too low (or cost too high), then the specialty will not want to propose even if $\theta=1$; if bias is too high (or cost too low), then the specialty will want to propose even if $\theta=0 .{ }^{50}$

\footnotetext{
${ }^{48}$ The argument inside of the angular distance formula is called the cosine similarity, or $\cos \left(\sigma_{A}, \sigma_{B}\right)$. Denoting Euclidean distance between $\sigma_{A}$ and $\sigma_{B}$ as $\left\|\sigma_{A}-\sigma_{B}\right\|_{2},\left\|\sigma_{A}-\sigma_{B}\right\|_{2}^{2}=\left(\sigma_{A}-\sigma_{B}\right)\left(\sigma_{A}-\sigma_{B}\right)^{\prime}=\left\|\sigma_{A}\right\|_{2}^{2}+\left\|\sigma_{B}\right\|_{2}^{2}-$ $2 \sigma_{A} \cdot \sigma_{B}$. If $\left\|\sigma_{A}\right\|_{2}^{2}=\left\|\sigma_{B}\right\|_{2}^{2}=1$, then $\left\|\sigma_{A}-\sigma_{B}\right\|_{2}^{2}=2\left(1-\cos \left(\sigma_{A}, \sigma_{B}\right)\right)$. In Figure A-1, we find that in our data, Euclidean distance and weighted Euclidean distance are quite similar, while the other metrics tend to shrink larger distances.

${ }^{49}$ We could introduce these features, but the intuition we wish to formalize would remain the same.

${ }^{50}$ Potters and van Winden (1992) also point out that there exists a mixed strategy equilibrium, in which
} 
We then consider two specialties $S \in\{1,2\}$, and assume that $b_{S}>\frac{c-1}{2}$ for both specialties. Both specialties would propose if $\theta=1$ had the other one not existed, yet neither would propose if it knows that the other specialty's strategy is to propose when $\theta=1$. Thus, there is no unique pure strategy equilibrium of proposals by the two specialties. In the case that $b_{1}=b_{2}$, this implies that there is no symmetric pure strategy equilibrium. ${ }^{51}$ There are at least two types of mixed strategies over the range of this bias-cost space: (i) Neither specialty proposes if $\theta=0$ and mix (i.e., propose with some probability $\left.\pi_{S} \in(0,1)\right)$ if $\theta=1$, and (ii) both specialties propose if $\theta=1$ and mix if $\theta=0$.

Because the number of actual specialty-proposals relative to potential specialty-proposals is empirically low, we focus on the former type of mixed strategies. ${ }^{52}$ When specialties mix when $\theta=1$, the RUC knows that $\theta=1$ and sets $p=1$, if either $D_{1}=1$ or $D_{2}=1$. If $D_{1}=D_{2}=0$, the RUC sets

$$
p\left(\pi_{1}, \pi_{2} \mid D_{1}=D_{2}=0\right)=\frac{\left(1-\pi_{1}\right)\left(1-\pi_{2}\right)}{1+\left(1-\pi_{1}\right)\left(1-\pi_{2}\right)},
$$

which is the probability that $\theta=1$ if $D_{1}=D_{2}=0$. For a mixed-strategy equilibrium to exist, specialties must be indifferent between proposing and not. In Figure A-2, fixing $c=1$ for the specialties, we show whether a mixed-strategy equilibrium exists in $\left(b_{1}, b_{2}\right)$ space and, if so, the mixing probabilities for the specialties (when $\theta=1$ ) that sustain it.

We first find that if specialty bias is sufficiently low, then there exists no mixed-strategy equilibrium. Failure to coordinate and the temptation to free-ride results in no proposals, reducing signaling equilibria relative to the one-specialty standard. However, if both specialties are sufficiently biased (or equivalently, have low costs), then their equilibrium mixed strategies will involve fairly high $\pi_{S}$, and signaling is possible even when it was not in the one-specialty case (due to bias being too high relative to costs). Finally, and intuitively, when specialty are asymmetric in their bias, signaling occurs mostly through the lower-bias specialty. As the bias of the higher-bias specialty approaches infinity, the equilibrium resembles a single-specialty purestrategy equilibrium and is possible at levels of bias (of the lower-bias specialty) close to those in the one-specialty case (i.e., as low as $\frac{c-1}{2}$ ).

the specialty certainly proposes if $\theta=1$ and proposes with some probability $\pi \in(0,1)$ if $\theta=0$ for biases $b_{S} \in\left(\frac{c+1}{2}, c+\frac{1}{4}\right)$ if $c>\frac{1}{2}$.

${ }^{51}$ There may exist, for example, a perfect Bayesian equilibrium with two players in which both would choose to propose if the RUC believed $\theta=1$ only if it observes both specialties proposing. We could rule out an equilibrium of this form by refining the equilibrium concept such that if the RUC observes only one specialty proposing, it will nonetheless consider that specialty's incentives to propose and update its prior probability that the value of $\theta=1$ (Grossman and Helpman, 2001).

${ }^{52}$ Parallel results obtain for the latter type, which correspond to the mixed strategy in the single-specialty case noted by Potters and van Winden (1992), in footnote 50. 


\section{A-4 Specialty-Proposal Propensity and Simulated Affiliation}

Our measure of affiliation is determined mechanically by specialties on the RUC, $\mathcal{R}_{t}$, and specialties on a proposal, $\mathcal{S}_{i}$. To assess our identifying assumption - that affiliation is uncorrelated with underlying price, conditional on observable characteristics of a proposal - we examine the probability of specialty participation in proposals, as this is an endogenous object. As we discuss in Section 3.3, the key intuition for our assumption on affiliation is that a variety of factors determine the specialty cost of proposing and that, with many potential proposers and costly proposing, there exist multiple pure- and mixed-strategy equilibria.

In Figure 6, we show evidence that actual specialty proposals are uncorrelated with predicted price, conditional on specialty-proposal propensities. Originally, we estimate proposal propensities for each specialty using a logit model, conditioning on specialty identities and functions of specialty shares of the procedure's utilization $\mathbf{w}_{i}$. Here, we enrich our propensity model to (i) show substantial residual variation in proposals and (ii) form a basis to simulate affiliation. Furthermore, we will use simulated measures of affiliation to (iii) demonstrate unbiasedness in our prediction of affiliation based on proposal propensities and (iv) use predicted (mean simulated) affiliation as a control for robustness.

We first estimate a logit propensity model of specialty-proposal participation, using specialty identities, flexible functions of $\mathbf{w}_{i}$, and the procedure's share of specialty revenue, defined as $\sigma_{i s}^{R}$ in Appendix A-2.1.2. While the logit model is fairly predictive, with a pseudo- $R^{2}$ of 0.73 and a log-likelihood of $-8,661.35$ over 248,735 observations, we find substantial residual variation in specialty proposals. To illustrate this, in Figure A-3, we show the propensities of 6,929 actual specialty-proposal pairs over 4,199 proposals. While there are many propensities with high values, more than half of the actual specialty proposals have propensities lower than 0.8 , and about a quarter have propensities less than 0.5. Similarly, in Figure A-4, we show the first, second-, third-, and fourth-ranked specialty propensities for proposals with at least as many proposers. Although there are 64 specialties to rank, propensities quickly diminish: The average first-ranked propensity is 0.86 , while the average second-, third-, and fourth-ranked propensities are $0.76,0.69$, and 0.54 , respectively.

Based on estimated specialty-proposal propensities, $\hat{\pi}_{i s}$, and the known specialties of RUC members at each meeting, $\mathcal{R}_{t}$, we can simulate affiliation. We proceed as follows:

1. Use estimated specialty-proposal propensities, $\hat{\pi}_{i s}$. Drop any specialty-proposal pair with $\hat{\pi}_{i s}<0.01$.

2. For each proposal $i$, identify number of remaining specialty-proposer candidates, $n_{i}$, and the number of actual specialty proposers, $k_{i}$. This yields $\left\|\mathbb{S}_{i}\right\|=C\left(n_{i}, k_{i}\right)$ as the number of potential proposer sets $\mathcal{S}_{i}$ for $i$, constraining the number of simulated proposers in each set to be the same as the number of actual proposers. For example, if there remain ten

specialty-proposer candidates for a proposal with only one actual specialty proposer, there 
are $C(10,1)=10$ (singleton) sets to draw from. However, if there are fifteen specialtyproposer candidates for a proposal with four actual proposers, there are $C(15,4) \approx 7.57 \times$ $10^{7}$ sets to draw from.

(a) For proposals $i$ such that $\left\|\mathbb{S}_{i}\right\| \leq 50$, collect all such potential proposer sets.

(b) For the remaining proposals, randomly draw $k_{i}$ proposers from $n_{i}$, oversampling specialty-proposer candidates from those with higher $\hat{\pi}_{i s}$. Specifically, generate $r_{i s} \sim$ $U(0,1)$ and subtract this from $\hat{\pi}_{i s}$. Within each $i$, sort specialty-proposer candidates by $\hat{\pi}_{i s}-r_{i s}$, and choose the top $k_{i}$ candidates to include in $\mathcal{S}_{i}$. Repeat until some stopping rule (e.g., based on the number of unique sets sampled for each $i$ and the lack of new sets sampled for any $i$ in a draw).

(c) Denote as $\mathbb{S}_{i}^{*} \subseteq \mathbb{S}_{i}$ the collection of simulated sets for each proposal $i$. For each $\mathcal{S}_{i} \in \mathbb{S}_{i}^{*}$, calculate a simulated set affiliation $A\left(\mathcal{R}_{t}, \mathcal{S}_{i}\right)$ for each $\mathcal{S}_{i}$, using known $\mathcal{R}_{t}$ and the formula in Equation (4).

3. Given $\hat{\pi}_{i s}$, and assuming independence of specialty proposals, the probability of drawing $\mathcal{S}_{i}$ from $\mathbb{S}_{i}$ is

$$
\operatorname{Pr}\left(\mathcal{S}_{i} \mid \mathbb{S}_{i}^{*}\right) \equiv \frac{\prod_{s \in \mathcal{S}_{i}} \hat{\pi}_{i s} \prod_{s \notin \mathcal{S}_{i}}\left(1-\hat{\pi}_{i s}\right)}{\sum_{\mathcal{S}_{i} \in \mathbb{S}_{i}^{*}}\left(\prod_{s \in \mathcal{S}_{i}} \hat{\pi}_{i s} \prod_{s \notin \mathcal{S}_{i}}\left(1-\hat{\pi}_{i s}\right)\right)} .
$$

This allows us to weight sets by their probability of occurrence. This also allows us to generate a predicted set affiliation,

$$
\hat{A}\left(\mathcal{R}_{t}, i\right)=\sum_{\mathcal{S}_{i} \in \mathbb{S}_{i}^{*}} A\left(\mathcal{R}_{t}, \mathcal{S}_{i}\right) \operatorname{Pr}\left(\mathcal{S}_{i} \mid \mathbb{S}_{i}^{*}\right)
$$

In Figure A-5, we show the distribution of simulated set affiliations relative to the actual set affiliation for each $i$, weighted by $\operatorname{Pr}\left(\mathcal{S}_{i} \mid \mathbb{S}_{i}^{*}\right)$. The weighted standard deviation of the distribution is 0.242 , reflecting that there exists meaningful variation in set affiliation based on the specialtyproposal propensities. Further, the weighted mean of the distribution is -0.015 , suggesting very little forecast bias in predicted set affiliation. We use predicted set affiliation as a control, rather than linear specialty shares of CPT utilization, $\mathbf{w}_{i}$, in a robustness check of the affiliation effect on prices, in Column 5 of Table 3; we find a similar estimate of the main effect.

\section{A-5 Counterfactual Revenue Simulation Algorithm}

We simulate counterfactual revenue in scenarios that entail counterfactual affiliations for proposals. In each scenario, we hold fixed the service and timing of each proposal, the Medicare budget, and the utilization of each service. Counterfactual revenue results solely from the effect of affiliation on relative price. Prices are rationalized so that total spending meets the fixed Medicare budget. The algorithm is as follows: 
1. Starting at the first year in which the RUC's pricing decision goes into effect, we replace the relative price $R V U_{i y}$ that followed a RUC recommendation with a counterfactual $\widetilde{R V U}_{i y}$, by subtracting $\hat{\alpha}\left(A\left(\mathcal{R}_{t}, \mathcal{S}_{i}\right)-\tilde{A}\left(\mathcal{R}_{t}, \mathcal{S}_{i}\right)\right)$, where $A\left(\mathcal{R}_{t}, \mathcal{S}_{i}\right)$ and $\tilde{A}\left(\mathcal{R}_{t}, \mathcal{S}_{i}\right)$ are actual and counterfactual affiliations, respectively, and $\hat{\alpha}$ is the estimated affiliation effect from Equation (6). RUC decisions in meeting $t$ map to prices in the Medicare schedule in year $y(t)$. We repeat for subsequent years, allowing previously set prices to continue forward.

2. We take quantities $q_{i s y}$ of service $i$, by specialty $s$, in year $y$, observed in Medicare claims. We set conversion factors $C F_{y}$ and $\widetilde{C F}$ so that the overall spending is $\$ 70$ billion in 2014 dollars, which implies that

$$
\widetilde{C F}_{y} \sum_{i} \sum_{s} \widetilde{R V U}_{i y} \cdot q_{i s y}=C F_{y} \sum_{i} \sum_{s} R V U_{i y} \cdot q_{i s y} .
$$

3. The revenue reallocation for service $i$, specialty $s$, and year $y$ is

$$
\Delta r_{i s y}=q_{i s y}\left(\widetilde{C F}_{y} \cdot \widetilde{R V U}_{i y}-C F_{y} \cdot R V U_{i y}\right) \text {. }
$$

4. We aggregate $\Delta r_{i s y}$ to yearly averages for specialties $s$ or types of service $k$ :

$$
\begin{aligned}
\Delta R_{s} & =\|\mathcal{Y}\|^{-1} \sum_{y \in \mathcal{Y}} \sum_{i} \Delta r_{i s y} \\
\Delta R_{k} & =\|\mathcal{Y}\|^{-1} \sum_{y \in \mathcal{Y}} \sum_{s} \sum_{i \in k} \Delta r_{i s y} .
\end{aligned}
$$

\section{A-6 Alternative Mechanisms Behind the Price Effect}

In this appendix, we consider evidence regarding alternative mechanisms of the affiliation effect on prices, summarized in Table A-4. All specifications in Table A-4 include the same controls as in the baseline specification of the price regression, shown in Column 4 of Table 3.

First, we consider the possibility that affiliation could reflect signaling "buy-in." That is, more specialties should be willing to propose for procedures that have a higher intrinsic price. As more specialties propose, set affiliation, as defined in Equation (4), will mechanically increase through max operator in the formula. Higher prices under this scenario are warranted and do not reflect any RUC bias. We modify the baseline price-effect regression in Equation (6) to include proposer-count fixed effects:

$$
\ln \mathrm{RVU}_{i t}=\alpha A\left(\mathcal{R}_{t}, \mathcal{S}_{i}\right)+\gamma_{n} \mathbf{1}\left(\left\|\mathcal{S}_{i}\right\|=n\right)+\mathbf{X}_{i} \beta+\mathbf{T}_{t} \eta+\mathbf{w}_{i} \zeta+\varepsilon_{i t} .
$$

This specification relies only on within-proposer-count variation to identify the price effect of affiliation. As shown in Column 1 of Table A-4, the coefficient on standardized set affiliation is 
unchanged, at 0.112 , and highly significant.

Second, we consider a range of specifications relating to interests (and information) held by RUC specialties that are specific to the proposed service. Specifically, we consider the service $i$ 's utilization share of all services billed by a RUC specialty $s$, or $\sigma_{i s}^{q}$, as defined in Equation (2) and discussed in Appendix A-2.1.1. We also consider $i$ 's revenue share of all Medicare revenue to $s$, or $\sigma_{i s}^{R}$, as defined and discussed in Appendix A-2.1.2. These measures capture both proposal-specific interests and information about $i$. We perform variants of the regression

$$
\ln \mathrm{RVU}_{i t}=\alpha A\left(\mathcal{R}_{t}, \mathcal{S}_{i}\right)+\gamma m_{s \in \mathcal{R}_{t}}\left(\boldsymbol{\sigma}_{i}\right)+\mathbf{X}_{i} \beta+\mathbf{T}_{t} \eta+\mathbf{w}_{i} \zeta+\varepsilon_{i t}
$$

where $m_{s \in \mathcal{R}_{t}}\left(\boldsymbol{\sigma}_{i}\right)$ is a moment function of the vector of service-specific utilization or revenue shares for specialties serving on the RUC, $s \in \mathcal{R}_{t}$.

Columns 2 and 3 of Table A-4 show results for regressions adding standardized mean $\sigma_{i s}^{q}$ and $\sigma_{i s}^{R}$, respectively. Columns 4 and 5 show results for regressions adding standardized 33rd percentile $\sigma_{i s}^{q}$ and $\sigma_{i s}^{R}$, respectively, to reflect the two-thirds vote count required for passing a proposal before the RUC. In all specifications, the coefficient on standardized set affiliation remains unchanged in magnitude and significance. The coefficients on the standardized measures of RUC-specialty interest in proposal $i$ are small, though statistically significant. Although we ascribe a causal interpretation to the effect of affiliation, given our discussion in Section 3.3, the same reasoning does not hold for $\gamma .{ }^{53}$

\section{A-7 Technical Details of the Conceptual Framework}

This appendix provides additional detail behind the conceptual framework we outline in Section 5. We start with more detail about the formula for expected "variance", $E\left[\left(\theta+b_{R}-p\right)^{2}\right]$, that represents information loss in the standard Crawford and Sobel (1982) model. Next, we provide details of the analysis with hard information and the optimal $b_{R}^{*}$ under hard information. Finally, we describe a mechanism of assigning intervals of expected length $L$ such that the posterior distribution of $\theta$ remains uniform within each realized interval.

\section{A-7.1 Canonical Crawford and Sobel (1982) Partitions}

Consider $\theta$ uniformly distributed on the interval $[0, L]$. The sender (the specialty) has bias $b$, relative to the receiver (the RUC). The formula for the number of partitions supported under

\footnotetext{
${ }^{53}$ For $\gamma$ to have a causal interpretation, we would require random variation in RUC specialty composition, $\mathcal{R}_{t}$. As we discuss in Section 3.3, the RUC has had a relatively fixed composition over time, compared to variation in specialty proposals. In contrast, note that $m_{s \in \mathcal{R}_{t}}\left(\boldsymbol{\sigma}_{i}\right)$ is invariant to the specialty identities in the proposal, $\mathcal{S}_{i}$.
} 
$b=b_{S}-b_{R}$ over the interval is

$$
n^{*}(b)=\left\lfloor\frac{1}{2}\left(1+\sqrt{1+\frac{2 L}{b}}\right)\right\rfloor .
$$

Using Equation (A-7.7), we define the limiting bias such that $n^{*}(b-\varepsilon)=n$ for any positive but arbitrarily small $\varepsilon$ :

$$
b^{*}(n)=\frac{2 L}{(2 n-1)^{2}-1} .
$$

$b^{*}(n)$ supports $n$ partitions only in the limit. For example, as we show below, $b=\frac{1}{4}$ supports only one partition, since the first partition of technically two partitions will have a length of 0 .

The first partition is bounded by $x_{0}=0$ and

$$
x_{1}=\frac{L}{n}-(n-1) 2 b,
$$

Subsequent partition lengths increase by $4 b$, which implies

$$
x_{k}=2 x_{k-1}-x_{k-2}+4 b,
$$

and Equations (A-7.8) and (A-7.9) imply that $x_{n}=L$.

We will consider a number of specific examples of $n$, which exist for $b \in\left[b^{*}(n+1), b^{*}(n)\right)$. We define the boundaries of the partitions in the space of $[0, L]$, and the variance $E\left[\left(\theta+b_{R}-p\right)^{2}\right]$. For the latter object, we use the fact that the variance of a uniformly distributed random variable along an interval of length $L$ is $L^{2} / 12$. Two partitions exist if $b \in\left[\frac{L}{12}, \frac{L}{4}\right)$ and are defined by $\left(0, \frac{L}{2}-2 b, L\right)$. The variance is given by

$$
\begin{aligned}
E\left[\left(\theta+b_{R}-p\right)^{2}\right] & =\frac{L^{2}}{12}\left[\left(\frac{1}{2}-2 b\right)^{3}+\left(\frac{1}{2}+2 b\right)^{3}\right] \\
& =\frac{1}{12}\left(\frac{L^{2}}{4}+12 b^{2}\right)=\frac{L^{2}}{48}+b^{2}
\end{aligned}
$$

Three partitions exist if $b \in\left[\frac{L}{24}, \frac{L}{12}\right)$ and are defined by $\left(0, \frac{L}{3}-4 b, \frac{2 L}{3}-4 b, L\right)$. The variance is given by

$$
\begin{aligned}
E\left[\left(\theta+b_{R}-p\right)^{2}\right] & =\frac{L^{2}}{12}\left[\left(\frac{1}{3}-\frac{4 b}{L}\right)^{3}+\left(\frac{1}{3}\right)^{3}+\left(\frac{1}{3}+\frac{4 b}{L}\right)^{3}\right] \\
& =\frac{1}{12}\left(\frac{L^{2}}{9}+32 b^{2}\right) .
\end{aligned}
$$


By induction, one can verify that the variance in the equilibrium with $n$ partitions is

$$
E\left[\left(\theta+b_{R}-p\right)^{2}\right]=\frac{1}{12}\left(\frac{L^{2}}{n^{2}}+A_{n} b^{2}\right)
$$

where $A_{1}=0$, and $A_{n}=A_{n-1}+8 n-4$. Note that the variance is continuous across the number of partitions (as $b$ changes). Also, the variance is decreasing in $b$, holding $L$ fixed.

\section{A-7.2 Hard Information}

Given the formula for soft information loss in Equation (A-7.10), we can write the expected utility for the specialty and the government, respectively, as

$$
\begin{aligned}
E\left[u_{S}\right] & =-E\left[\left(\theta+\theta_{R}-p\right)^{2}\right]-b^{2}-c(L) \\
& =-\frac{1}{12}\left(\frac{L^{2}}{n^{2}}+A_{n} b^{2}\right)-b^{2}-c(L),
\end{aligned}
$$

and as

$$
\begin{aligned}
E\left[u_{G}\right] & =-E\left[\left(\theta+\theta_{R}-p\right)^{2}\right]-b_{R}^{2} \\
& =-\frac{1}{12}\left(\frac{L^{2}}{n^{2}}+A_{n} b^{2}\right)-b_{R}^{2} .
\end{aligned}
$$

In both Equations (A-7.11) and (A-7.12), $n$ is the number of partitions supported by $b$ and $L$ and is given by Equation (A-7.7). Better information, either hard or soft, increases the utility of both the specialty and the government.

Taking the partial derivative of expected specialty utility with respect to $L$, while holding $b$ and $n$ fixed, yields the following condition for the agent's choice of $L$ :

$$
\frac{\partial}{\partial L} E\left[u_{S}\right]=-\frac{L}{6 n^{2}}-c^{\prime}(L)=0 .
$$

The convexity of $c(L)$ implies that there exists a single optimal candidate that satisfies Equation (A-7.13) for the cheap talk equilibrium with $n$ partitions. Denote the solution to Equation (A7.13) for a given $n$, if it exists (i.e., $n^{*}\left(b, L_{n}^{*}\right)=n$ ), as $L_{n}^{*} .{ }^{54}$ Intuitively, $L_{n}^{*}$ is increasing in $n$ : better soft communication (higher $n$ ) reduces the incentive to produce hard information (larger $\left.L_{n}^{*}\right)$. The globally optimal $L^{*}$ is then given by $L^{*}=\arg \max _{n}\left(E\left[u_{S} ; L_{n}^{*}\right]\right) . L^{*}$ is decreasing in $b$ : As the specialty and the RUC have divergent preferences, soft communication worsens, and this increases the optimal hard information. Because the set of $L_{n}^{*}$ is comprised of discrete values, $L^{*}(b)$ is a step function.

\footnotetext{
${ }^{54}$ In Equation (A-7.13), $L_{n}^{*}$ is increasing in $n$, and in Equation $(\mathrm{A}-7.7), n^{*}(b, L)$ is increasing in $L$. Since (i) $L_{n}^{*} \in(0,1]$ and (ii) $n^{*}(b, L)$ is bounded by $n^{*}(b, 1)$, there must be at least one $n \in\left\{1, \ldots, n^{*}(b, 1)\right\}$ such that $n^{*}\left(b, L_{n}^{*}\right)=n$.
} 


\section{A-7.3 Optimal RUC Bias}

Because smaller $L^{*}$ increases government utility in Equation (A-7.12), and because $L^{*}$ is a decreasing function of $b=b_{R}-b_{S}$, the optimal $b_{R}^{*}$ from the government's perspective is weakly lower under the possibility of hard information than when we fix $L=1$.

However, the optimal $b_{R}^{*} \geq 0$. That is, an adversarial RUC is still never optimal from the government's perspective. In order for $b_{R}^{*}<0$, we need three requirements:

1. The threshold $\bar{b}_{R}$ where the specialty is indifferent between $n=1$ and $n=2$ must be less than 0 .

2. The expected government utility when $b_{R}=\bar{b}_{R}$ is higher than the maximum expected government utility under $n=2$ :

$$
\max E\left[u_{G} \mid n=2\right]<E\left[u_{G} \mid b_{R}=\bar{b}_{R}\right]
$$

3. The expected government utility when $b_{R}=\bar{b}_{R}$ is higher than complete delegation when $b_{R}=b_{S}$.

Note also that convexity of $c(L)$ implies that $c^{\prime}\left(L_{1}^{*}\right)<c^{\prime}\left(L_{2}^{*}\right)$. From the first order conditions that $c^{\prime}\left(L_{1}^{*}\right)=-\frac{1}{6} L_{1}^{*}$ and $c^{\prime}\left(L_{2}^{*}\right)=-\frac{1}{24} L_{2}^{*}$, we must have $L_{1}^{*}>\frac{1}{4} L_{2}^{*}$. Convexity also implies that

$$
\frac{c\left(L_{1}^{*}\right)-c\left(L_{2}^{*}\right)}{L_{2}^{*}-L_{1}^{*}} \in\left[\frac{1}{24} L_{2}^{*}, \frac{1}{6} L_{1}^{*}\right] .
$$

The threshold $\bar{b}_{R}$ is defined by the following condition:

$$
E\left[u_{G} \mid b_{R}=\bar{b}_{R}, n=1\right]=E\left[u_{G} \mid b_{R}=\bar{b}_{R}, n=2\right] .
$$

In other words

$$
\frac{1}{12}\left(L_{1}^{*}\right)^{2}+\left(\bar{b}_{R}-b_{S}\right)^{2}+c\left(L_{1}^{*}\right)=\frac{1}{48}\left(L_{2}^{*}\right)^{2}+2\left(\bar{b}_{R}-b_{S}\right)^{2}+c\left(L_{2}^{*}\right) .
$$

The threshold is then

$$
b_{S}=\bar{b}_{R}+\sqrt{\frac{1}{12}\left(L_{1}^{*}\right)^{2}-\frac{1}{48}\left(L_{2}^{*}\right)^{2}+c\left(L_{1}^{*}\right)-c\left(L_{2}^{*}\right)} .
$$

Condition 1 and convexity imply that

$$
b_{S}<\sqrt{\frac{1}{12}\left(L_{1}^{*}\right)^{2}-\frac{1}{48}\left(L_{2}^{*}\right)^{2}+\frac{1}{6} L_{1}^{*}\left(L_{2}^{*}-L_{1}^{*}\right)}
$$


Condition 2 requires that

$$
-\frac{1}{12}\left(L_{1}^{*}\right)^{2}-\bar{b}_{R}^{2}>-\frac{1}{48}\left(L_{2}^{*}\right)^{2}-\frac{1}{2} b_{S}^{2},
$$

where the expression on the left is the expected government utility at $\bar{b}_{R}$ and $n=1$, and the expression on the right is the expected government utility under the optimal $b_{R}=\frac{1}{2} b_{S}$ conditional on $n=2$. Condition 3 requires that

$$
-\frac{1}{12}\left(L_{1}^{*}\right)^{2}-\bar{b}_{R}^{2}>-b_{S}^{2} .
$$

The expression on the right is the government utility under full delegation.

We show numerically that there are no values $\left(L_{1}^{*}, L_{2}^{*}, b_{S}, \bar{b}_{R}\right)$ that satisfy Equations (A-7.14) to (A-7.17) simultaneously.

\section{A-7.4 Uniform Posterior Intervals}

While it is convenient to work with continuous $L$, there is a technical complication in specifying values of $\underline{\theta}$ and $\bar{\theta}$, such that it remains that $\theta \sim U(\underline{\theta}, \bar{\theta})$ with fixed $L=\bar{\theta}-\underline{\theta}$. For example, consider the case of $L=0.9$. If $\theta=0$, then we must have $\underline{\theta}=0$ with probability 1 , but $\underline{\theta}=0$ with probability less than 1 if $\theta>0$. Therefore, if any potential interval must have $L=0.9$, and we have a realized interval $[\underline{\theta}, \bar{\theta}]=[0,0.9]$, then $\theta$ cannot be uniformly distributed within the realized interval.

To preserve uniform posterior distributions within the intervals revealed after hard information, we need sets of potential intervals to be mutually exclusive and collectively exhaustive. Thus, we may have one potential interval of length $L_{a}=0.9$ and another potential interval of length $L_{b}=0.1$. The ordering of these intervals may be random, but so long as the intervals are not overlapping in a particular ordering, then the posterior distribution of $\theta$ within each interval will remain uniform. We operationalize this with the concept that $L$ instead represents the expected length of the information interval after hard information, under a mechanism that divides the unit interval into $N$ intervals of length $L_{a}$ and a remaining weakly shorter interval of length $L_{b}=1-N L_{a} \leq L_{a}$.

The probability that $\theta$ falls in an interval of length $L_{a}$ is $N L_{a}$, while the probability that $\theta$ falls in the remaining interval of length $L_{b}$. Thus $L=N L_{a}^{2}+L_{b}^{2}=N L_{a}^{2}+\left(1-N L_{a}\right)^{2}$. We can solve for $L_{a}(L)$, as a function of $L$, by using the quadratic formula and the fact that $N=\left\lfloor L^{-1}\right\rfloor$ :

$$
L_{a}(L)=\frac{1+\sqrt{1-(1-L)\left(\left\lfloor L^{-1}\right\rfloor^{-1}+1\right)}}{1+\left\lfloor L^{-1}\right\rfloor},
$$

which is continuous and monotonically increasing in $L$.

We modify our equilibrium analysis by stating expected utility $E\left[u_{A}\right]$ (prior to hard infor- 
mation) as a function of $L$ :

$$
\begin{aligned}
E\left[u_{A}\right] & =-E\left[(\theta-p)^{2}\right]-b^{2}-c(L) \\
& =-\frac{1}{12}\left[\frac{N L_{a}^{3}}{n_{a}^{2}}+\frac{\left(1-N L_{a}\right)^{3}}{n_{b}^{2}}+\bar{A} b^{2}\right]-b^{2}-c(L),
\end{aligned}
$$

where $n_{a}=n^{*}\left(b, L_{a}\right), n_{b}=n^{*}\left(b, 1-N L_{a}\right) \leq n_{a}$, and $\bar{A}=N L_{a} A_{n_{a}}+\left(1-N L_{a}\right) A_{n_{b}}$. The expression for the variance $E\left[(\theta-p)^{2}\right]$ is continuous, monotonically increasing in $L\left(\right.$ and $\left.L_{a}(L)\right)$, and piecewise convex in $L_{a}(L)$. The remainder of the analysis proceeds by identifying solutions $L_{\mathbf{n}}^{*}$, where $\mathbf{n}=\left(n_{a}, n_{b}\right)$, and choosing $L^{*}=\arg \max _{\mathbf{n}}\left(E\left[u_{S} ; L_{\mathbf{n}}^{*}\right]\right)$.

\section{A-8 Private Price Transmission Robustness}

In Section 5.3, we show that private insurance price changes are more responsive to Medicare price changes when the Medicare price changes originate from RUC decisions and, within RUC decisions, when they originate from a higher-affiliation proposal. We interpret this finding as supporting the hypothesis that RUC decisions, particular those from higher-affiliation proposals, contain valuable information that private insurance follows. In this appendix, we investigate alternative mechanisms that may generate this result.

First, affiliated proposals may result in more informative Medicare prices not because they facilitate communication, as detailed in Section 5, but because RUC members may naturally have more information about the procedures that their specialty societies perform. We investigate this possibility by using proxy measures of the RUC members' own information, based on their utilization of the service in question. In particular, we consider a specialty $s$ 's share of total utilization for service $i, w_{i s}$, as defined in Equation (5), and the service $i$ 's share of the total utilization for specialty $s$, as defined in (2), averaging across the specialties of RUC members at the relevant meeting:

$$
\begin{aligned}
\bar{w}_{i y} & =\frac{1}{\left\|\mathcal{R}_{t(i, y)}\right\|} \sum_{s \in \mathcal{R}_{t(i, y)}} w_{i s} ; \\
\bar{\sigma}_{i y} & =\frac{1}{\left\|\mathcal{R}_{t(i, y)}\right\|} \sum_{s \in \mathcal{R}_{t(i, y)}} \sigma_{i s}
\end{aligned}
$$

where $\mathcal{R}_{t(i, y)}$ is the set of RUC member specialties at the meeting $t(i, y)$ corresponding to service $i$ and (private) price change year $y$.

Second, affiliated proposals may be for high-volume services for which both private insurers and Medicare have interests in setting accurate prices. Strong correlation between private insurance and Medicare price changes for high-affiliation proposals may then result from careful price-setting in both private insurance and Medicare, and not because affiliation per se causes 
better communication between proposing specialties and the RUC. We consider two measures of volume for service $i$ : private insurance volume and total (private insurance and Medicare) volume.

Third, we take an omnibus approach, agnostic to what in particular may drive greater price following from Medicare to private insurance, by fitting a predictive model of price following. We consider changes in private insurance prices as a function of changes in Medicare prices:

$$
\Delta \ln \operatorname{Price}_{i, y}^{P}=\alpha+\beta_{i y} \Delta \ln \operatorname{Price}_{i, y^{M}(i, y)}^{M}+\varepsilon_{i y},
$$

where the goal is to predict $\beta_{i y} .{ }^{55}$ To operationalize this, as an approximation of $\beta_{i y}$, we take the ratio of demeaned $\Delta \ln$ Price $_{i, y}^{P}$ and demeaned $\Delta \ln$ Price $_{i, y^{M}(i, y)}^{M}$,

$$
\text { Ratio }_{i y}=\frac{\Delta \ln \text { Price }_{i, y}^{P}-\overline{\Delta \ln \text { Price }^{P}}}{\Delta \ln \text { Price }_{i, y^{M}(i, y)}^{M}-\overline{\Delta \ln \text { Price }^{M}}},
$$

where $\overline{\Delta \ln \text { Price }^{P}}$ and $\overline{\Delta \ln \text { Price }^{M}}$ are respective sample means of log private and Medicare price changes, weighted by Medicare volume. We then predict this ratio as a linear function of private insurance volume for $i$; total (private insurance and Medicare) volume for $i$; time dummies $\mathbf{T}_{i y}$ for $y^{M}(i, y), y$, and RUC meeting; and the vector of specialty shares $\mathbf{w}_{i}$. We take the predicted ratio, $\widehat{\operatorname{Ratio}}_{i y}$, as an agnostic index for predicted price-following based on characteristics of $(i, y)$.

Given each of these measures that may influence price transmission to private insurance, we assess the robustness of our results to controlling for these measures, both directly and interacted with Medicare prices. Specifically, for each Index $i t$ measure (i.e., $\bar{w}_{i y}, \bar{\sigma}_{i y}$, private volume of $i$, total volume of $i$, and $\widehat{\operatorname{Ratio}}_{i y}$ ), we assess price transmission controlling for these proxy measures directly and interacted with Medicare prices, $\sigma_{i s}$, in regressions similar to Equation (8):

$$
\begin{aligned}
\ln \operatorname{Price}_{i y}^{P}= & \sum_{C}\left(\alpha_{C}+\beta_{C} \ln \operatorname{Price}_{i, y^{M}(i, y)}^{M}\right) \cdot \mathbf{1}(C(i, y)=C)+ \\
& \sum_{\tau=1}^{3}\left(\gamma_{\tau}+\delta_{\tau} \ln \operatorname{Price}_{i, y^{M}(i, y)}^{M}\right) \cdot \mathbf{1}\left(F\left(\operatorname{Index}_{i y}\right) \in\left(\frac{\tau-1}{3}, \frac{\tau}{3}\right)\right)+ \\
& \mathbf{T}_{i y} \eta+\xi_{i}+\varepsilon_{i y},
\end{aligned}
$$

where $\tau \in\{1,2,3\}$ indicates the tercile, $F(\cdot)$ is the distribution function of the relevant measure Index $i t$, and the rest is the same as in Equation (8). Table A-6 shows results from these regressions. The key coefficients of interest, $\beta_{C}$, are highly stable regardless of Index $i y$. Price transmission remains greater for Medicare price changes originating from RUC decisions and, within these decisions, from high-affiliation proposals.

\footnotetext{
${ }^{55}$ This changes-on-changes specification closely matches the fixed-effects specification in Equation (8). As shown in Figure A-8, separating Medicare price changes into high- and low-affiliation groups gives similar results.
} 


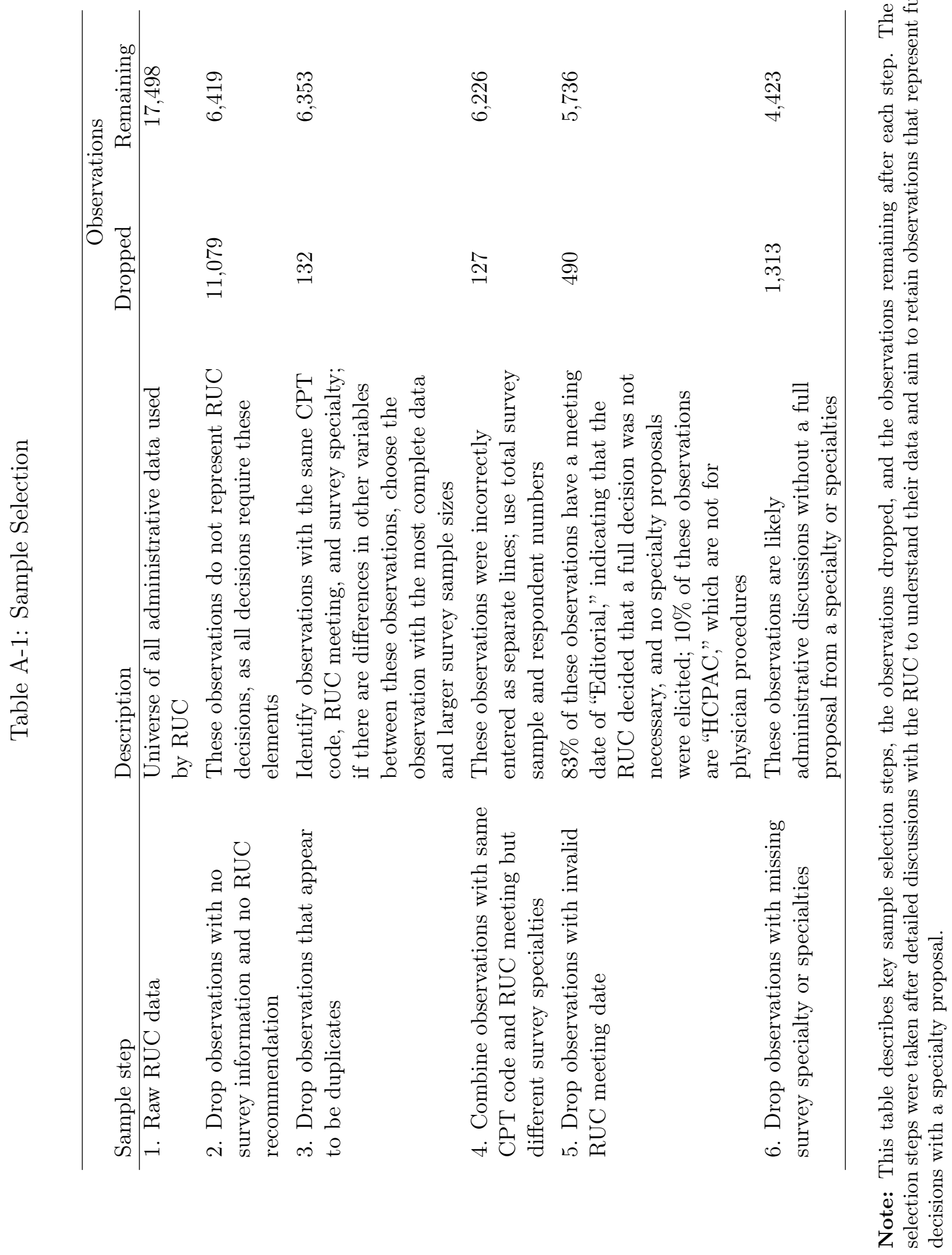




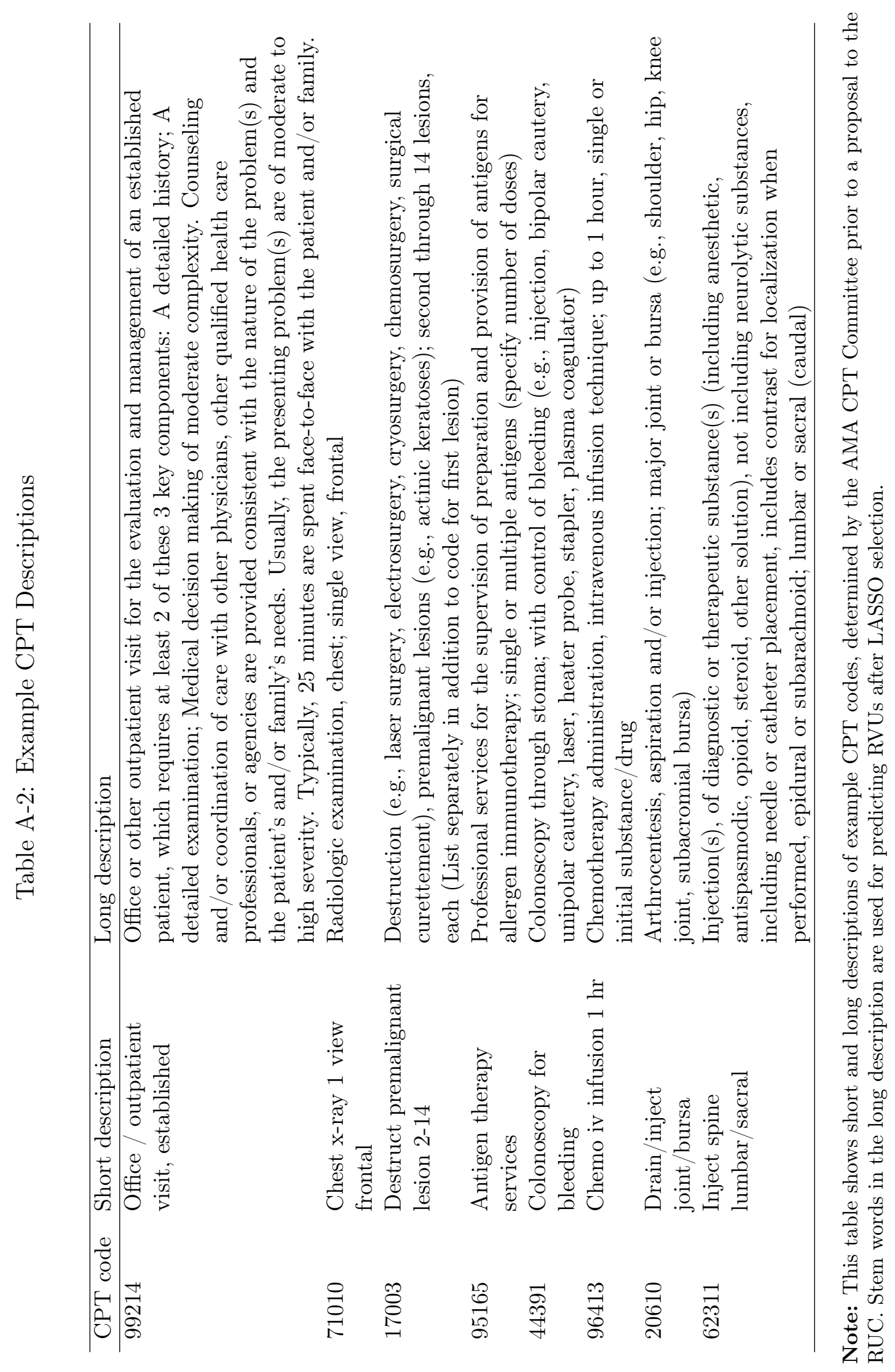


Table A-3: Price Effect of Alternative Affiliation Measures

\begin{tabular}{|c|c|c|c|c|c|}
\hline \multirow[b]{3}{*}{ Affiliation vector space } & $(1)$ & $(2)$ & $(3)$ & $(4)$ & $(5)$ \\
\hline & \multicolumn{5}{|c|}{ Affiliation metric } \\
\hline & Euclidean & $\begin{array}{c}\text { Gini- } \\
\text { Euclidean }\end{array}$ & Manhattan & Correlation & Angular \\
\hline & \multicolumn{5}{|c|}{ Panel A: Mean affiliation } \\
\hline Medicare CPT quantity & $\begin{array}{c}0.101^{* * *} \\
(0.029)\end{array}$ & $\begin{array}{c}0.104^{* * *} \\
(0.030)\end{array}$ & $\begin{array}{c}0.055^{* *} \\
(0.021)\end{array}$ & $\begin{array}{c}0.061^{* *} \\
(0.025)\end{array}$ & $\begin{array}{c}0.059^{* *} \\
(0.022)\end{array}$ \\
\hline $\begin{array}{l}\text { Medicare }+ \text { Marketscan } \\
\text { CPT quantity }\end{array}$ & $\begin{array}{c}0.076^{* * *} \\
(0.025)\end{array}$ & $\begin{array}{c}0.079 * * * \\
(0.026)\end{array}$ & $\begin{array}{c}0.048^{* *} \\
(0.020)\end{array}$ & $\begin{array}{c}0.057^{* *} \\
(0.025)\end{array}$ & $\begin{array}{c}0.055^{* *} \\
(0.023)\end{array}$ \\
\hline Medicare CPT revenue & $\begin{array}{c}0.094^{* * *} \\
(0.029)\end{array}$ & $\begin{array}{c}0.094^{* * *} \\
(0.029)\end{array}$ & $\begin{array}{l}0.038^{*} \\
(0.019)\end{array}$ & $\begin{array}{l}0.036^{*} \\
(0.021)\end{array}$ & $\begin{array}{l}0.036^{*} \\
(0.020)\end{array}$ \\
\hline $\begin{array}{l}\text { Medicare BETOS } \\
\text { quantity }\end{array}$ & $\begin{array}{c}0.088^{* * *} \\
(0.029)\end{array}$ & $\begin{array}{c}0.089^{* * *} \\
(0.030)\end{array}$ & $\begin{array}{l}0.056^{* *} \\
(0.022)\end{array}$ & $\begin{array}{c}0.049 * * \\
(0.024)\end{array}$ & $\begin{array}{c}0.050 * * \\
(0.022)\end{array}$ \\
\hline \multirow[t]{2}{*}{$\begin{array}{l}\text { Medicare BETOS } \\
\text { revenue }\end{array}$} & $\begin{array}{c}0.072^{* * *} \\
(0.027)\end{array}$ & $\begin{array}{c}0.068 * * \\
(0.028)\end{array}$ & $\begin{array}{c}0.045^{* *} \\
(0.021)\end{array}$ & $\begin{array}{c}0.030 \\
(0.020)\end{array}$ & $\begin{array}{l}0.032^{*} \\
(0.019)\end{array}$ \\
\hline & \multicolumn{5}{|c|}{ Panel B: 33rd percentile affiliation } \\
\hline Medicare CPT quantity & $\begin{array}{c}0.104^{* * *} \\
(0.032)\end{array}$ & $\begin{array}{c}0.111^{* * * *} \\
(0.031)\end{array}$ & $\begin{array}{l}0.061^{* *} \\
(0.024)\end{array}$ & $\begin{array}{c}0.060^{* *} \\
(0.026)\end{array}$ & $\begin{array}{c}0.060^{* *} \\
(0.025)\end{array}$ \\
\hline $\begin{array}{l}\text { Medicare }+ \text { Marketscan } \\
\text { CPT quantity }\end{array}$ & $\begin{array}{c}0.076^{* * *} \\
(0.024)\end{array}$ & $\begin{array}{c}0.081^{* * *} \\
(0.026)\end{array}$ & $\begin{array}{c}0.062^{* *} \\
(0.023)\end{array}$ & $\begin{array}{c}0.052^{* *} \\
(0.024)\end{array}$ & $\begin{array}{c}0.050 * * \\
(0.023)\end{array}$ \\
\hline Medicare CPT revenue & $\begin{array}{c}0.089 * * * \\
(0.031)\end{array}$ & $\begin{array}{c}0.091^{* * *} \\
(0.033)\end{array}$ & $\begin{array}{l}0.039^{*} \\
(0.021)\end{array}$ & $\begin{array}{c}0.027 \\
(0.022)\end{array}$ & $\begin{array}{c}0.026 \\
(0.022)\end{array}$ \\
\hline $\begin{array}{l}\text { Medicare BETOS } \\
\text { quantity }\end{array}$ & $\begin{array}{c}0.086^{* *} \\
(0.033)\end{array}$ & $\begin{array}{c}0.093^{* * *} \\
(0.034)\end{array}$ & $\begin{array}{c}0.066^{* * *} \\
(0.025)\end{array}$ & $\begin{array}{l}0.051^{* *} \\
(0.025)\end{array}$ & $\begin{array}{c}0.053^{* *} \\
(0.025)\end{array}$ \\
\hline $\begin{array}{l}\text { Medicare BETOS } \\
\text { revenue }\end{array}$ & $\begin{array}{c}0.088^{* * *} \\
(0.029)\end{array}$ & $\begin{array}{c}0.083^{* * *} \\
(0.029)\end{array}$ & $\begin{array}{c}0.053^{* *} \\
(0.022)\end{array}$ & $\begin{array}{c}0.034^{* *} \\
(0.016)\end{array}$ & $\begin{array}{c}0.043^{* *} \\
(0.018)\end{array}$ \\
\hline
\end{tabular}

Note: This table shows results of regressions of log RVU on various measures of set affiliation. Each cell represents the coefficient on the affiliation measure in a separate regression, stated in Equation (6) and corresponding to the preferred specification of Column 4 in Table 3. Further details about the regression controls are given in the note for Table 3. Rows of the table correspond to the vector space upon which affiliation is calculated; all vector spaces are in shares (i.e., elements of the vector for each specialty sum to 1). Columns correspond to affiliation metrics between two specialties, discussed in Appendix A-2. Panel A calculates the set affiliation measure as the mean maximized specialty-pair affiliation, which is the default and is given in Equation (4). Panel B calculates the set affiliation measure as the 33rd percentile of the maximized specialty-pair affiliations. Standard errors, clustered by RUC meeting, are in parentheses; *** denotes significance at the $1 \%$ level. 
Table A-4: Alternative Mechanisms Behind Price Effect

\begin{tabular}{|c|c|c|c|c|c|}
\hline & (1) & $(2)$ & (3) & (4) & (5) \\
\hline & \multicolumn{5}{|c|}{ Log RVU } \\
\hline Standardized set affiliation & $\begin{array}{c}0.112^{* * *} \\
(0.043)\end{array}$ & $\begin{array}{c}0.098^{* * *} \\
(0.029)\end{array}$ & $\begin{array}{c}0.103^{* * *} \\
(0.030)\end{array}$ & $\begin{array}{c}0.098^{* * *} \\
(0.030)\end{array}$ & $\begin{array}{c}0.104^{* * *} \\
(0.030)\end{array}$ \\
\hline \multicolumn{6}{|l|}{$\begin{array}{l}\text { Standardized measures } \\
\text { of RUC-specialty interest }\end{array}$} \\
\hline Mean $\sigma_{i s}^{q}$ & & $\begin{array}{c}0.021^{* *} \\
(0.009)\end{array}$ & & & \\
\hline Mean $\sigma_{i s}^{R}$ & & & $\begin{array}{c}0.031^{* * *} \\
(0.007)\end{array}$ & & \\
\hline 33rd percentile $\sigma_{i s}^{q}$ & & & & $\begin{array}{c}0.028^{* * *} \\
(0.003)\end{array}$ & \\
\hline 33rd percentile $\sigma_{i s}^{R}$ & & & & & $\begin{array}{c}0.030^{* * *} \\
(0.004)\end{array}$ \\
\hline Baseline controls & Y & Y & Y & Y & Y \\
\hline Proposer count dummies & Y & $\mathrm{N}$ & $\mathrm{N}$ & $\mathrm{N}$ & $\mathrm{N}$ \\
\hline$N$ & 4,401 & 4,401 & 4,401 & 4,401 & 4,401 \\
\hline Adjusted $R$-squared & 0.891 & 0.891 & 0.895 & 0.891 & 0.895 \\
\hline Sample mean log RVU & 1.567 & 1.567 & 1.567 & 1.567 & 1.567 \\
\hline
\end{tabular}

Note: This table shows results of regressions of $\log$ RVU on standardized set affiliation, with the addition of controls to test robustness to alternative mechanisms. Column 1 tests robustness to the alternative mechanism of signaling "buy-in," controlling for proposer dummies, as in Equation (A-6.5). Columns 2 to 5 relate to alternative mechanisms of proposal-specific information held by RUC specialties. These specifications, given in Equation (A-6.6), control for proposal-specific measures of utilization or revenue shares by RUC specialties, or $\sigma_{i s}^{q}$ and $\sigma_{i s}^{R}$. Measures are moments (mean or 33rd percentile) in the distribution across RUC specialties and are standardized to have mean 0 and standard deviation 1 . Details are given in Appendix A-6. All specifications include controls in the baseline price-effect regression, in Column 4 of Table 3. Standard errors, clustered by RUC meeting, are in parentheses; ** denotes significance at the $5 \%$ level, and ${ }^{* * *}$ denotes significance at the $1 \%$ level. 
Table A-5: Specialty Seats on Counterfactual RUC

\begin{tabular}{lclc} 
Specialty & Seats & Specialty & Seats \\
\hline Anesthesiology & 2 & Obstetrics and Gynecology & 2 \\
Cardiology & 1 & Oncology & 1 \\
Emergency Medicine & 2 & Ophthalmology & 1 \\
Family Medicine & 4 & Orthopedic Surgery & 1 \\
Gastroenterology & 1 & Pediatrics & 2 \\
General Surgery & 1 & Psychiatry & 1 \\
Internal Medicine & 4 & Radiology & 1 \\
Neurology & 1 & & \\
\hline
\end{tabular}

Note: This table shows members of a counterfactual RUC, in which seats are assigned in proportion to the population of physicians in each specialty. The number of total seats is 25 , as it is in the current RUC. This RUC accommodates the 16 largest specialties; including specialties with fewer physicians would require a larger RUC. Many smaller specialties lack a seat in this RUC; compare this to the broader range of specialties that have some representation on the actual RUC over time in Table 1. Physician population numbers are from Table 1.1 of Association of American Medical Colleges (2016), accessible at https://www . aamc.org/data/workforce/reports/458480/1-1-chart.html. 


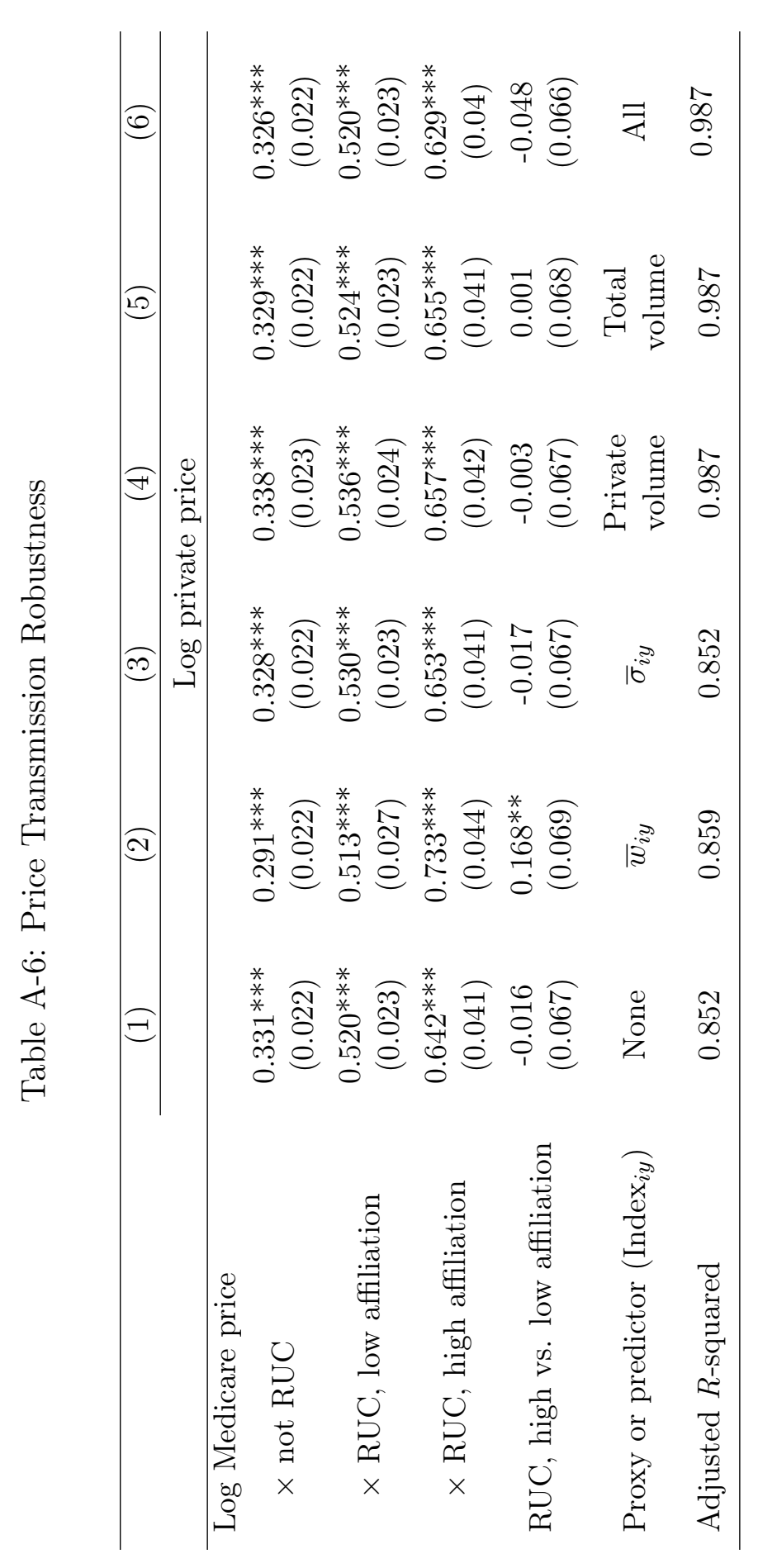

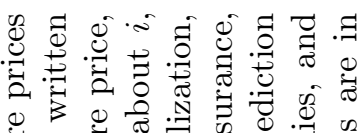

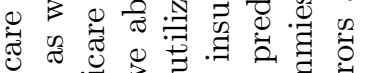
跣 20 要

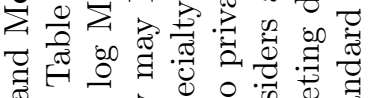

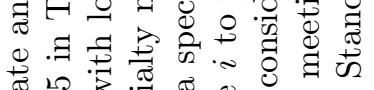

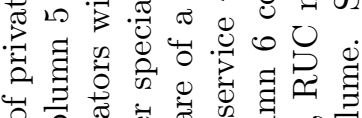
पे \%

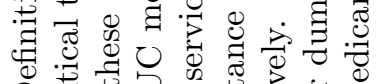

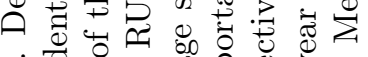
15.

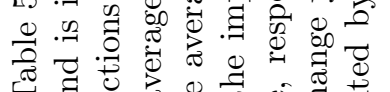

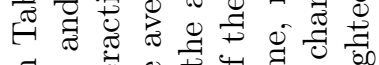

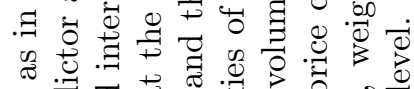
政 记 云

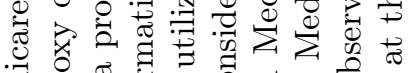

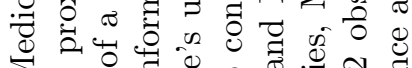

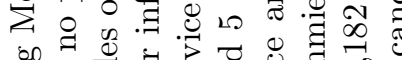

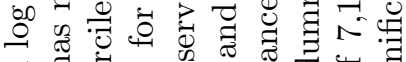
青 ष

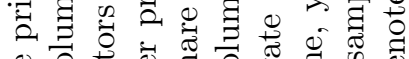

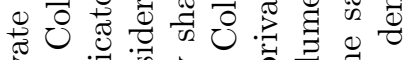

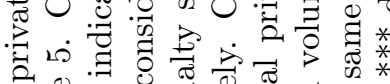

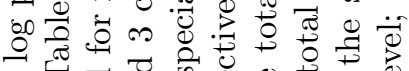
पू山

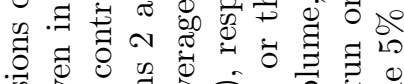

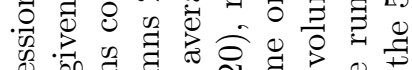

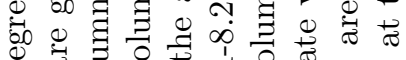

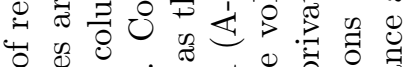

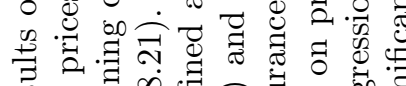
क्ष

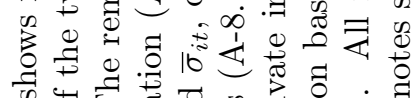
可

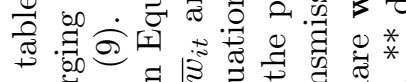

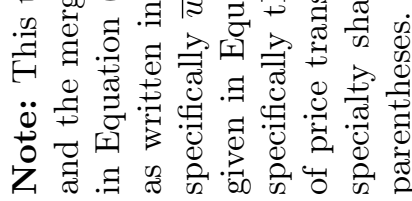


Figure A-1: Relationship between Affiliation Measures
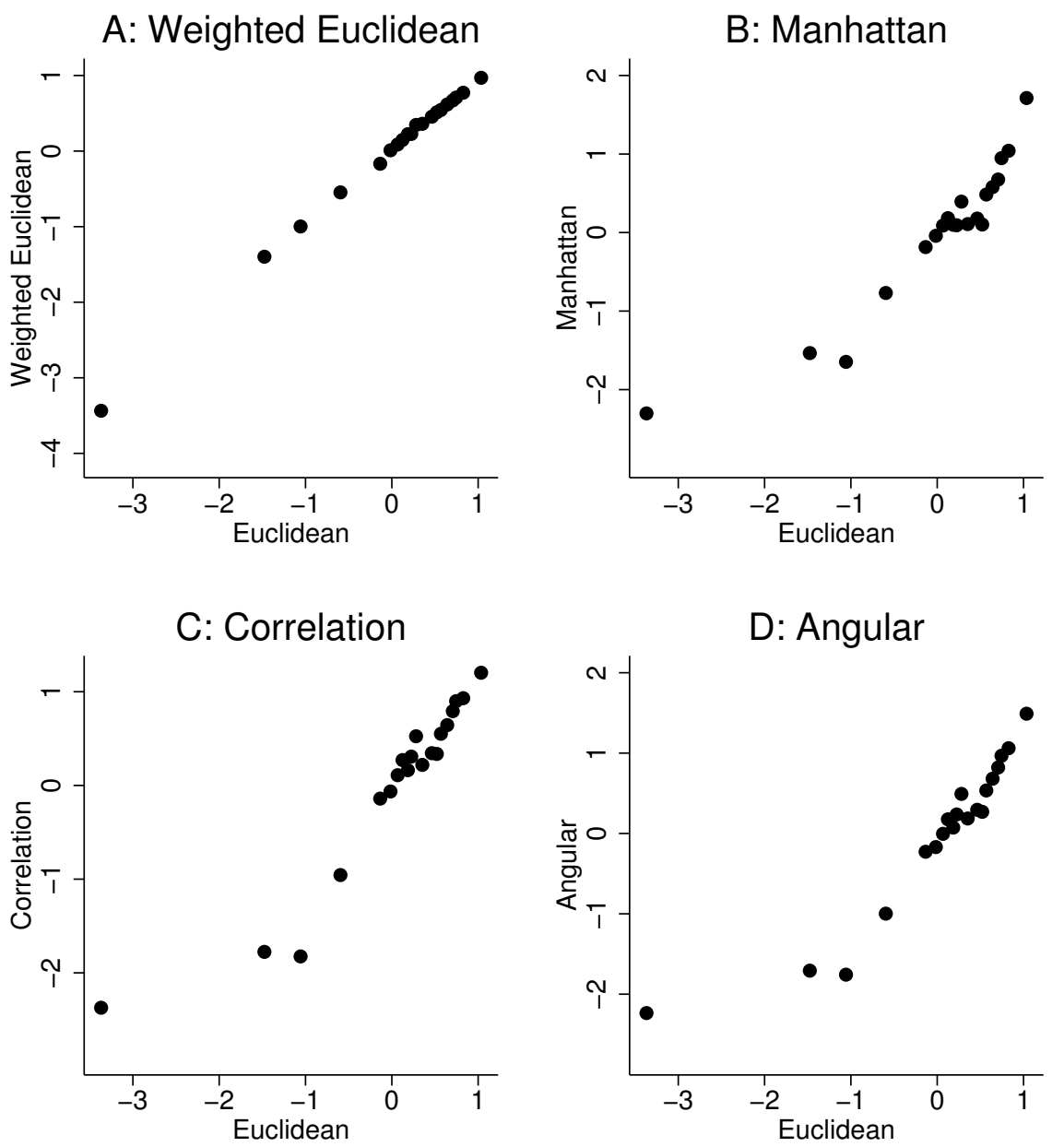

Note: This figure shows the relationship between different affiliation measures and the baseline measure of Euclidean distance. All measures are in the affiliation vector space of Medicare quantity shares. In each panel, the alternative affiliation measure is plotted against the Euclidean distance affiliation measure, for the sample of 4,423 proposals. Each dot represents a $5 \%$ sample of the data, ordered by the Euclidean distance affiliation measure, and averages of the respective affiliation measures are plotted. We provide further details on the definition of affiliation measures in Appendix A-2. 
Figure A-2: Mixed Strategy Proposal Probabilities

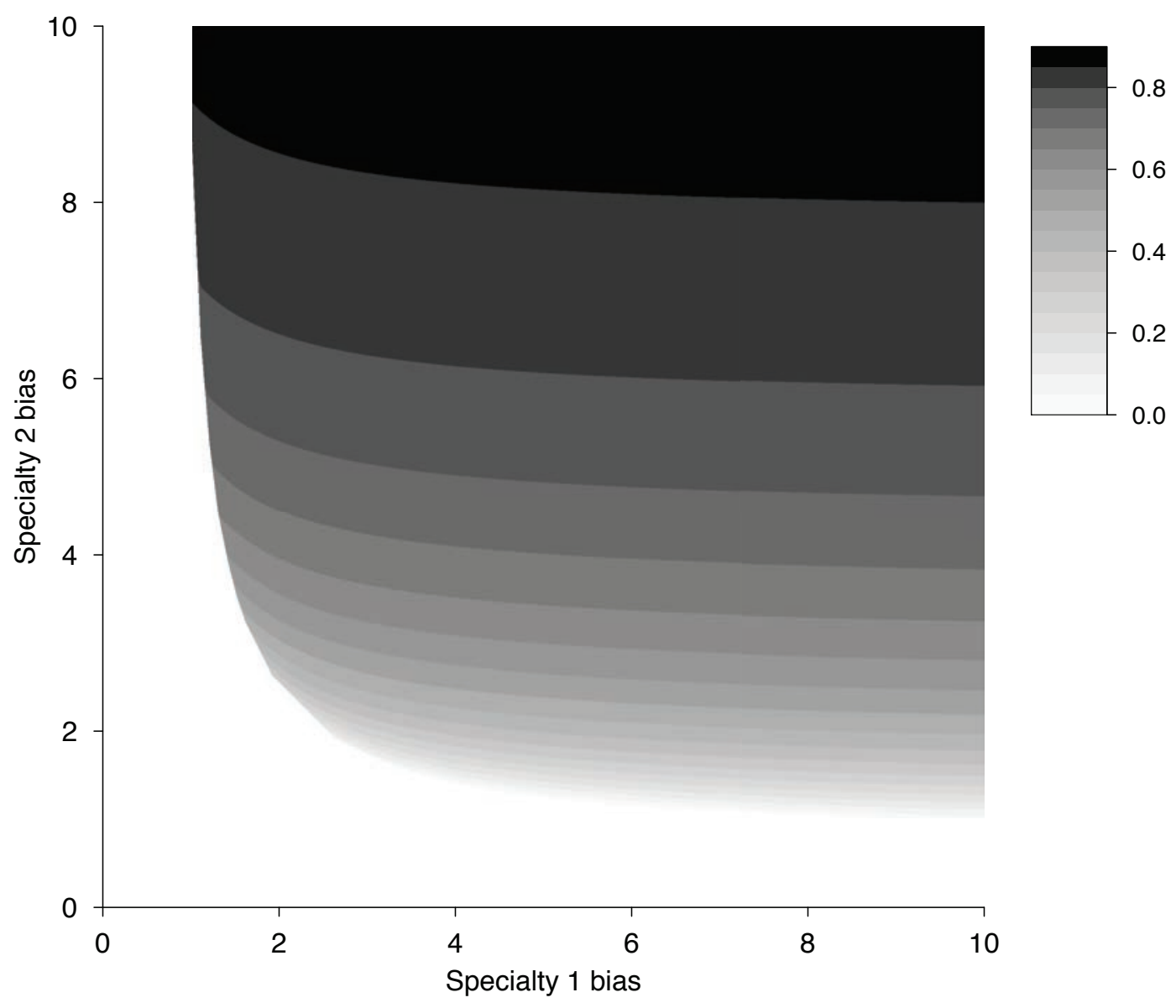

Note: This figure shows the probability of proposal participation under $\theta=1$ by specialty 1 in a mixed strategy equilibrium, in which specialties do not propose if $\theta=0$ and mix if $\theta=1$, described in Appendix A-3. Proposal probabilities are depicted in the space of bias by specialties 1 and 2. No mixed strategy equilibria exist in the region shown in pure white. 
Figure A-3: Distribution of Specialty-Proposal Propensities among Proposers

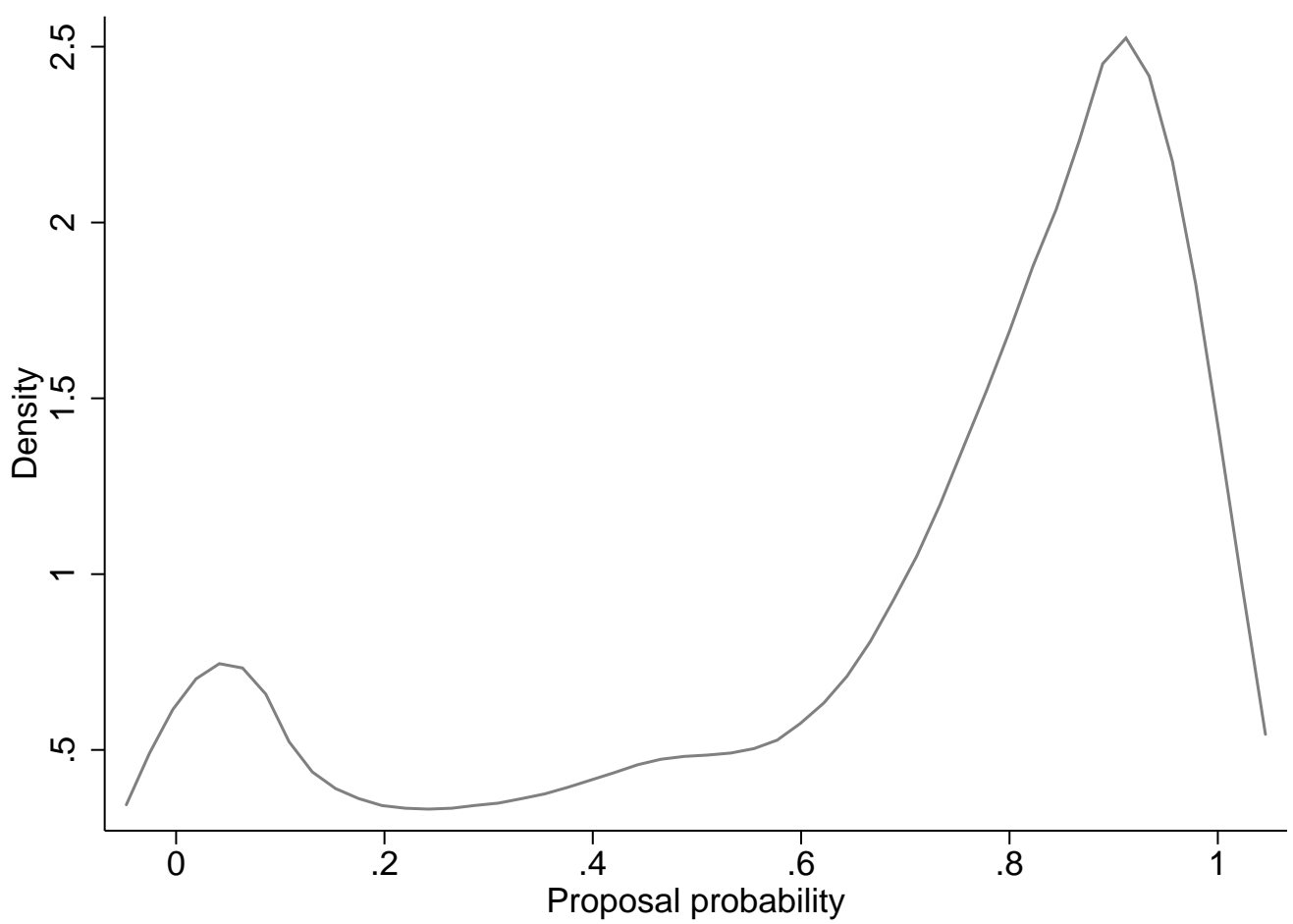

Note: This figure shows the density of specialty-proposal propensities, estimated by a logit model of 248,735 specialty-proposal pairs as described in Appendix A-4. Proposal propensities are shown only for 6,929 actual specialty-proposal pairs over 4,199 proposals. 
Figure A-4: Distribution of Highly Ranked Specialty-Proposal Propensities

A: First Rank

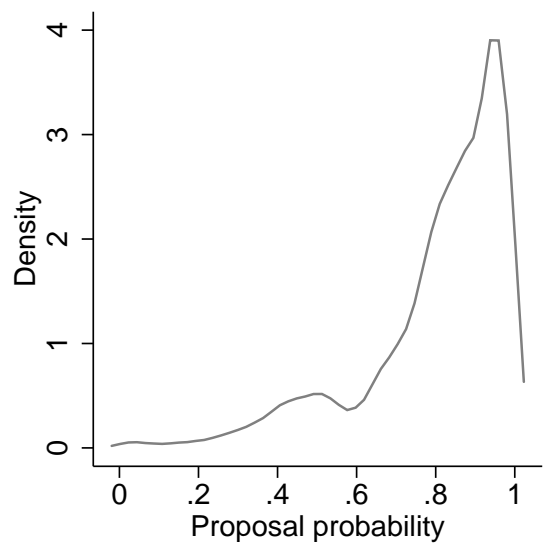

C: Third Rank

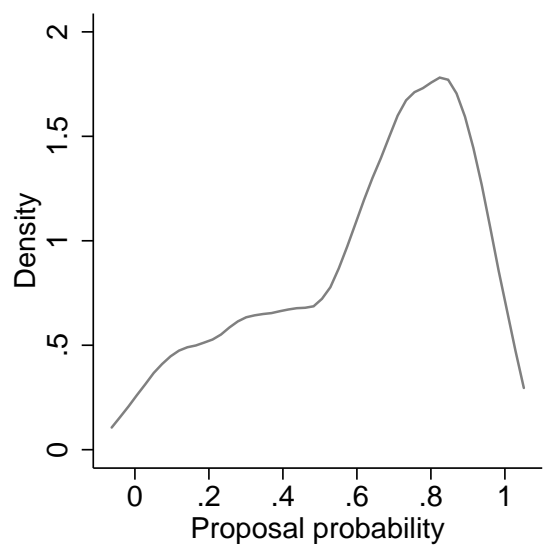

B: Second Rank

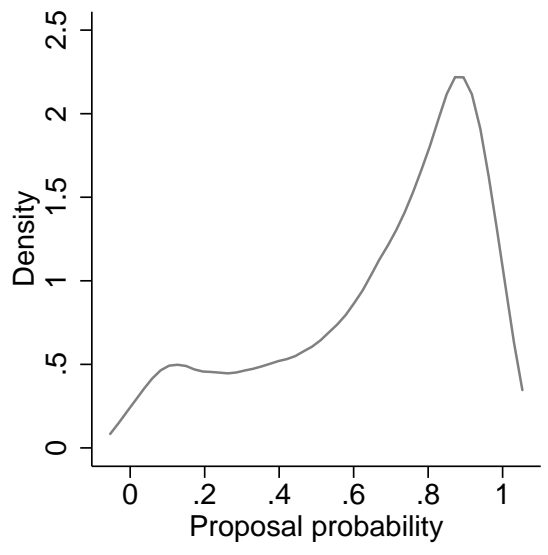

D: Fourth Rank

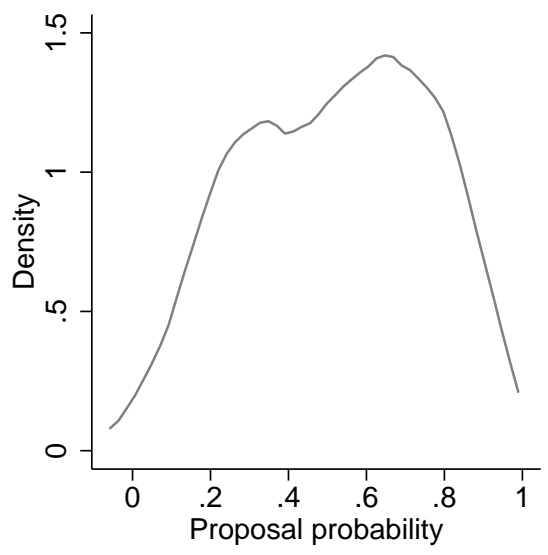

Note: This figure shows the density of specialty-proposal propensities, estimated by a logit model of 248,735 specialty-proposal pairs as described in Appendix A-4. In each panel, proposal propensities are shown only for correspondingly ranked specialty for proposals that have at least as many actual proposers. Specifically, in Panel A, the highest specialty propensity is shown for 4,199 proposals. In Panel B, the second-highest specialty propensity is shown for 1,524 proposals with at least two proposers. In Panel C, the third-highest specialty propensity is shown for 558 proposals with at least three proposers. In Panel $\mathrm{D}$, the fourth-highest specialty propensity is shown for 300 proposals with at least four proposers. 
Figure A-5: Distribution of Simulated Set Affiliation Relative to Actual Set Affiliation

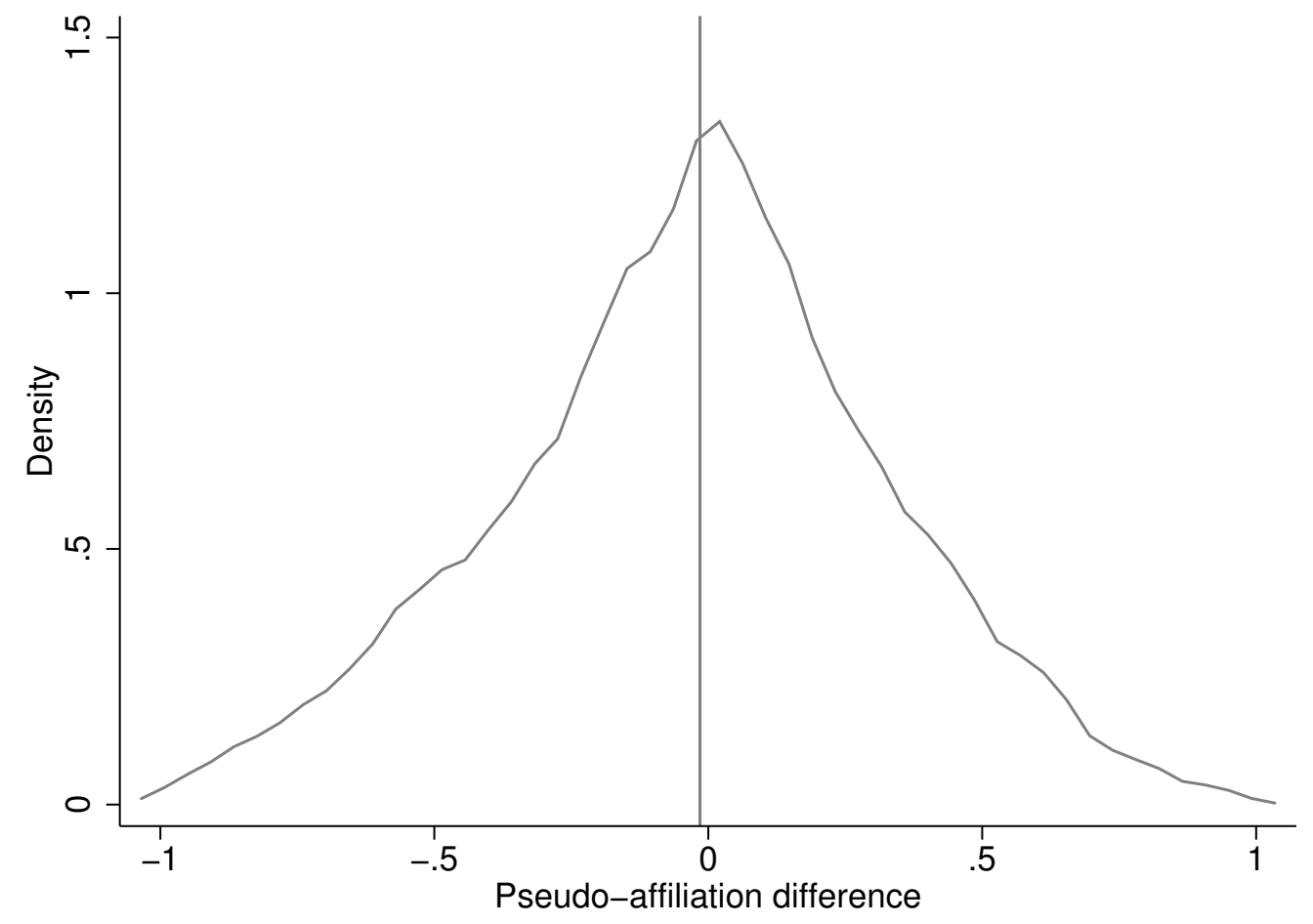

Note: This figure shows the density of 51,763 simulated set affiliations, using actual $\mathcal{R}_{t}$ and simulated proposing specialty sets $\mathcal{S}_{i}$ for each proposal $i$, differenced by actual set affiliation. Simulated specialtyproposals are derived from a logit model of specialty-proposal propensities, as illustrated in Figures A-3 and A-4. Simulated observations are weighted by their likelihood of being drawn. The weighted standard deviation of the simulated set affiliations is 0.242 , and the weighted mean of the differenced statistic is -0.015 . Details of the simulation algorithm are described in Appendix A-4. 

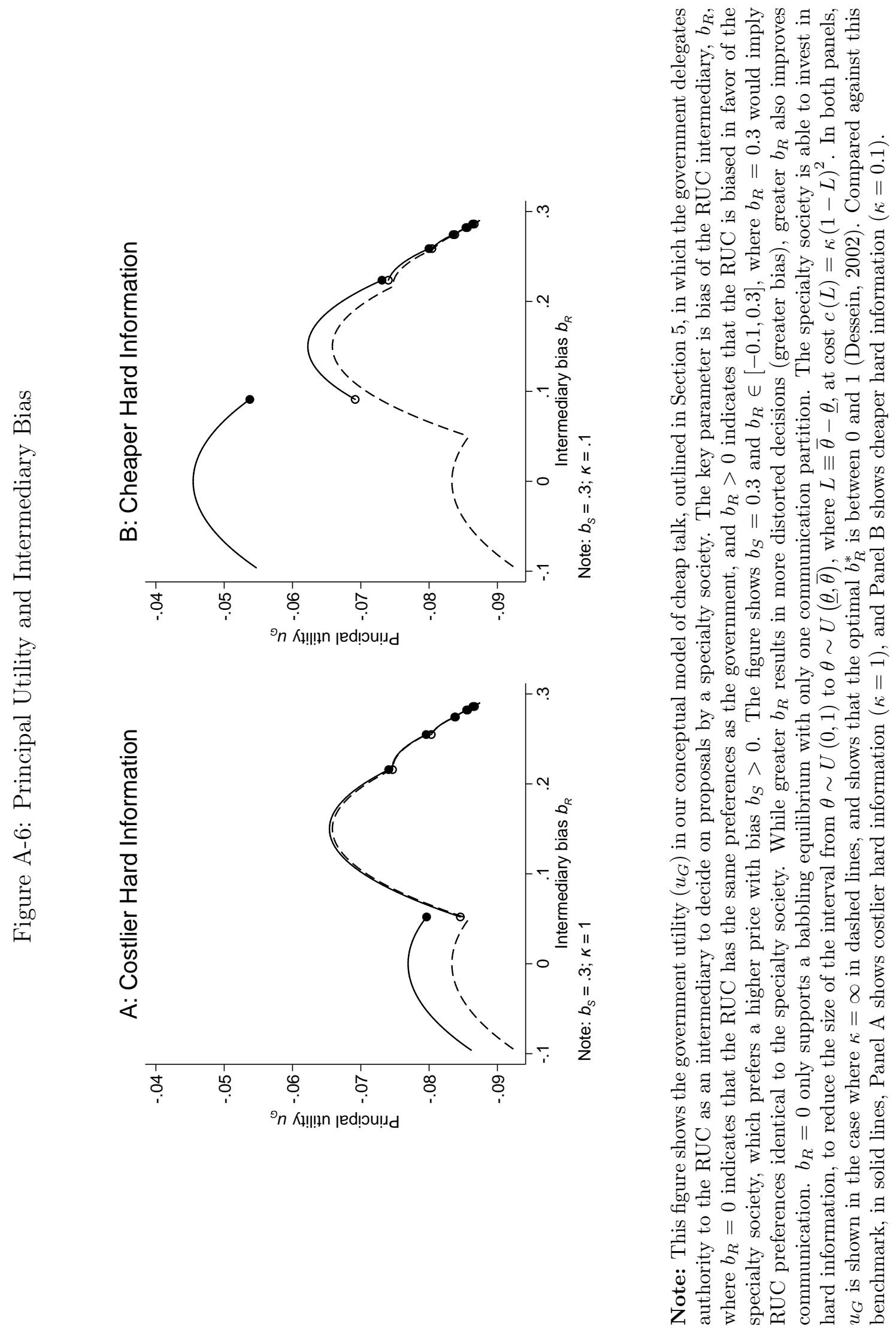


\section{Figure A-7: Distribution of Normalized Log Medicare Price Changes}

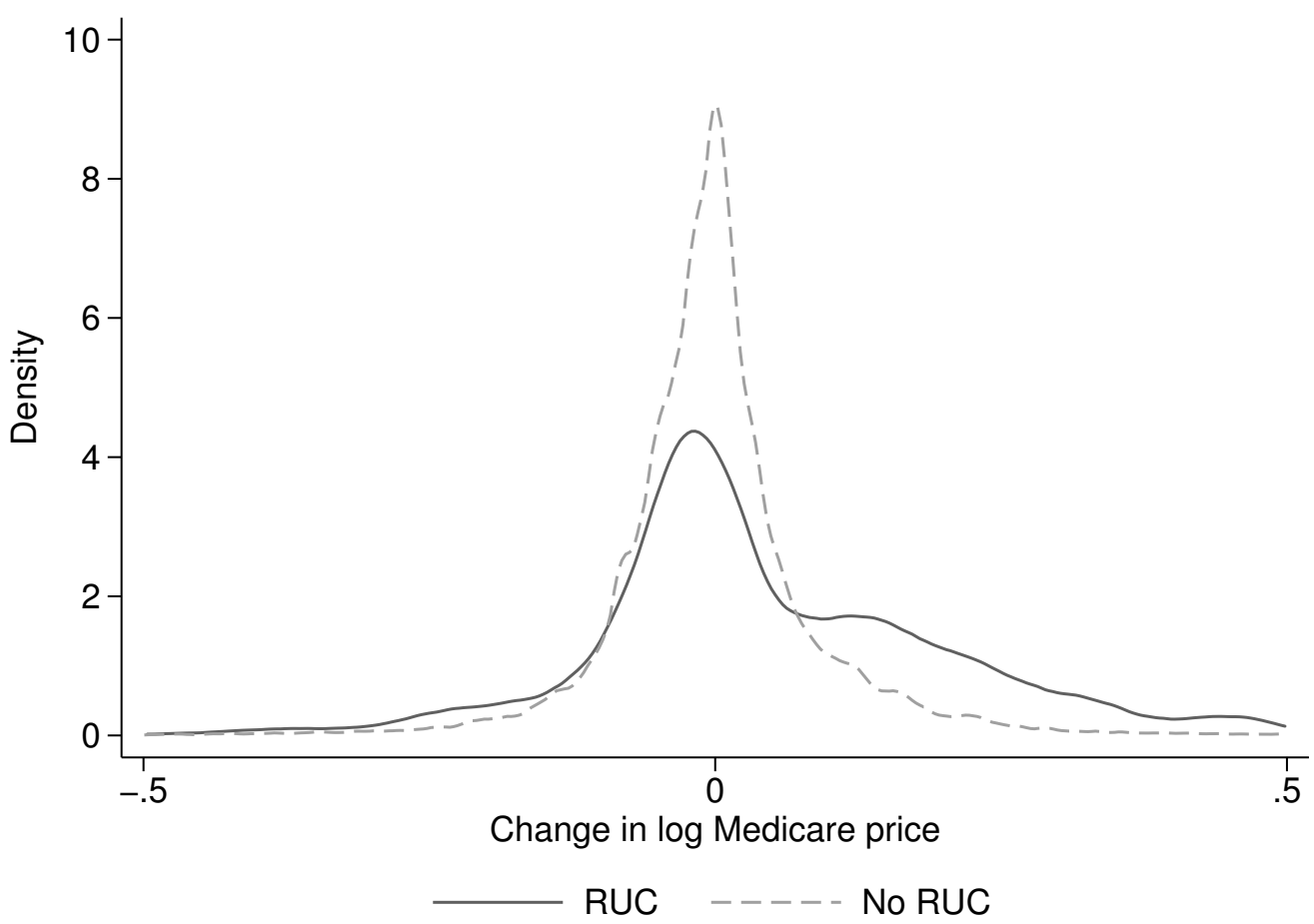

Note: This figure shows the density of Medicare price changes associated with a RUC decision (solid line) or not (dashed line). Medicare prices are defined as the total charges divided by the total volume of claims for each CPT code and year pair observed in the $100 \%$ sample of Medicare claims. The figure excludes any pair with fewer than 10 claims. Log prices are then normalized by subtracting the average $\log$ Medicare price across CPT codes in a given year, weighted by frequency of claims. The figure plots the difference between the normalized log price for a CPT code in a year and the price for the same CPT code in the previous year. 
Figure A-8: Private Price Changes on Medicare Price Changes

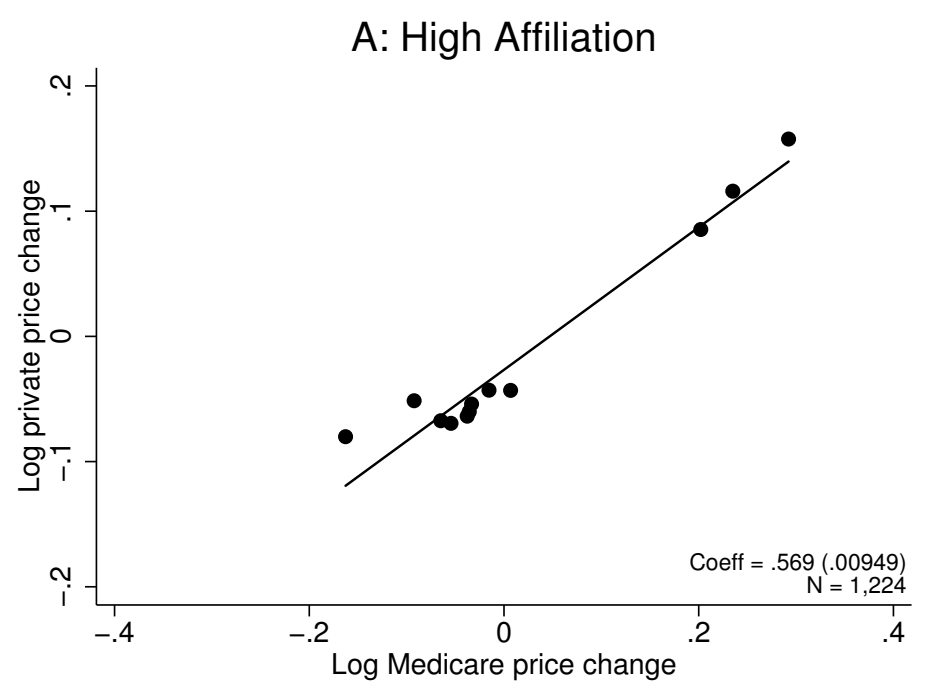

B: Low Affiliation

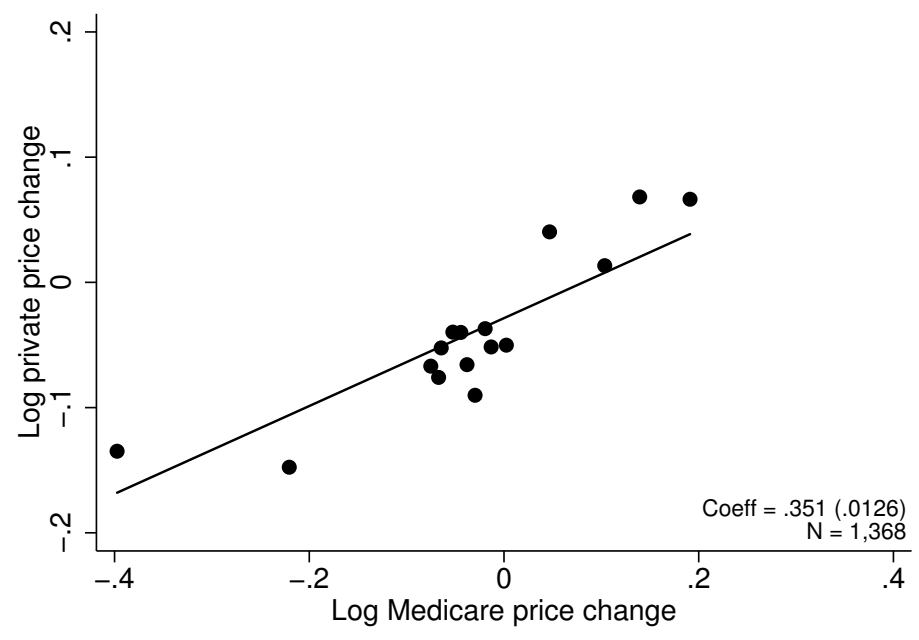

Note: This figure is a binned scatterplot of log private price changes on log Medicare price changes arising from high-affiliation RUC proposals (Panel A) and low-affiliation RUC proposals (Panel B), where each dot represents $5 \%$ of the data, ordered by Medicare price change. Lines show the best fit through the data, and the line slopes correspond to coefficients on log Medicare price change in a univariate regression of $\log$ private price change. Coefficients are robust to regression controls similar to those in Table 5. For consistency with Table 5, observations are weighted by frequency of Medicare claims for a given service (CPT code). Unweighted observations yield higher coefficients of approximately 1.5 for high-affiliation RUC proposals and 1 for low-affiliation RUC proposals. 